\title{
Gauge-invariant coherent states for loop quantum gravity: II. Non-Abelian gauge groups
}

\author{
Benjamin Bahr ${ }^{1}$ and Thomas Thiemann ${ }^{1,2}$ \\ ${ }^{1}$ MPI für Gravitationsphysik, Albert-Einstein Institut, Am Mühlenberg 1, 14467 Golm, Germany \\ 2 Perimeter Institute for Theoretical Physics, 31 Caroline St. N, Waterloo, Ontario N2L 2Y5, \\ Canada \\ E-mail: bbahr@aei.mpg.de and thomas.thiemann@aei.mpg.de
}

Received 4 August 2008, in final form 27 November 2008

Published 3 February 2009

Online at stacks.iop.org/CQG/26/045012

\begin{abstract}
This is the second paper concerning gauge-invariant coherent states for loop quantum gravity. Here, we deal with the gauge group $S U(2)$, this being a significant complication compared to the Abelian $U(1)$ case encountered in the previous article (Class. Quantum Grav. 26 045011). We study gauge-invariant coherent states on certain special graphs by analytical and numerical methods. We find that their overlap is Gauss peaked in gauge-invariant quantities, as long as states are not labeled by degenerate gauge orbits, i.e. points where the gauge-invariant configuration space has singularities. In these cases the overlaps are still concentrated around these points, but the peak profile exhibits a plateau structure. This shows how the semiclassical properties of the states are influenced by the geometry of the gauge-invariant phase space.
\end{abstract}

PACS numbers: $02.10 . \mathrm{Ox}, 02.40 . \mathrm{Vh}$, 04.60.Pp, 11.15.Kc

(Some figures in this article are in colour only in the electronic version)

\section{Introduction}

In [1], which is the first of a pair of papers, gauge-invariant coherent states for loop quantum gravity (LQG) for the Abelian gauge group $U(1)$ were considered. It was found that these states, which are defined by projecting the complexifier coherent states [2, 3] onto the gauge-invariant sub-Hilbert space, are labeled by points of the classical gauge-invariant phase space and a semiclassicality parameter, which encodes how well the state approximates this classical point. It was furthermore found that the overlap between two such states decreases exponentially, as the two labeling points become distinct. This showed that these states are promising tools for semiclassical approximations in the gauge-invariant sector of the theory. 
In the present paper, we will turn to the case $S U(2)$, which is the gauge group employed in full LQG. There, we aim to establish similar results as for $G=U(1)$. The plan for this paper is as follows: in section 2 we will briefly review the kinematical framework of LQG. After describing the Hilbert space and the action of the (Gauss) gauge transformations, we repeat the definition of the complexifier coherent states (CCS) of LQG in section 3.

In section 4, we will define the gauge-invariant coherent states as the projection of the complexifier coherent states onto the gauge-invariant Hilbert space. We show how the labels of these states can be interpreted as points in gauge-invariant phase space, and comment on the Ehrenfest properties of these states. Furthermore, we will investigate these states on some simple graphs, in particular the 1-flower, the 2-flower, the 3-bridge and the tetrahedron graph. In particular, we will compute the overlap between gauge-invariant coherent states on these graphs, demonstrating their peakedness properties. While on the 1-flower graph all calculations can be done analytically, the shape of the gauge orbits in the two other examples is already too complicated to allow for an analytical treatment of these cases. Rather, we will use numerical methods for investigating the overlap of gauge-invariant coherent states on these graphs, confirming the qualitative results about their peakedness properties from the 1-flower graph. To compute the overlap, in particular the inner product of the gauge-invariant coherent states accessible to these graphs, an algorithm is used to separate the gauge-dependent from the gauge-invariant degrees of freedom, which resembles a gauge-fixing procedure.

In section 5, we will work with a general formula of the inner product of gauge-invariant coherent states on arbitrary graphs, in order to establish some qualitative results about the peakedness properties of these states. Specifically, we will be able to relate the inner product of states on arbitrary graphs to those on flower graphs. Again, we will employ gauge-fixing methods on this behalf. This will allow for a qualitative description of the overlaps of gaugeinvariant coherent states labeled by degenerate gauge orbits.

We will close with a summary of the present work, as well as with a conclusion and an outlook.

\section{The kinematical setting of LQG}

We briefly repeat the kinematical framework of LQG. Detailed expositions can be found in [4-7] and in the references therein.

The starting point of LQG is the phase space of Ashtekar connections $A_{a}^{I}(x)$ and electric fluxes $E_{J}^{b}(y)$, both fields on a 3 -dim spatial manifold $\Sigma$, which can be thought of as a Cauchy surface in spacetime. The Poisson structure is given by

$$
\begin{aligned}
& \left\{A_{a}^{I}(x), A_{b}^{J}(y)\right\}=\left\{E_{I}^{a}(x), E_{J}^{b}(y)\right\}=0 \\
& \left\{A_{a}^{I}(x), E_{J}^{b}(y)\right\}=8 \pi G \beta \delta_{b}^{a} \delta_{J}^{I} \delta(x-y) .
\end{aligned}
$$

Here $G$ is Newton's constant, and $\beta$ is the Barbero-Immirzi parameter. The fields are not free, but subject to so-called constraints, which are phase-space functions, i.e. functions of $A_{a}^{I}(x)$ and $E_{J}^{b}(y)$. They encode the diffeomorphism invariance of the theory, and the Einstein equations. The reduced phase space consists of all phase-space points $A, E$ where the constraints vanish. On this set, the constraints act as gauge transformations, and the set of gauge orbits is the physical phase space. The set of constraints is divided into the Gauss constraints $G_{I}(x)$, the diffeomorphism constraints $D_{a}(x)$ and the Hamilton constraints $H(x)$. It is the set of Gauss constraints that is of particular importance in the rest of this work.

The holonomy-flux algebra generated by holonomies of $A_{a}^{I}(x)$ along edges and electric fields $E_{J}^{b}(y)$ smeared over 2-dim surfaces is the starting point of the quantization program. There is a unique cyclic representation of this algebra in which the spatial diffeomorphisms, 
which are generated by the diffeomorphism constraints $D_{a}(x)$, act unitarily and leave the vacuum state $\Omega$ invariant [8]. This kinematical Hilbert space $\mathcal{H}_{\text {kin }}$, on which the holonomyflux algebra is represented, also carries a representation of the constraint algebra, and is given by

$$
\mathcal{H}_{\text {kin }}=\bigoplus_{\gamma \in \Gamma} \mathcal{H}_{\gamma}
$$

Here, $\Gamma$ is the set of all graphs $\gamma$ in $\Sigma$ which consist of embedded, regular, analytic edges. Each Hilbert space $\mathcal{H}_{\gamma}$ is separable. If $\gamma$ is a graph with $E$ edges and $V$ vertices, $\mathcal{H}_{\gamma}$ is isomorphic to

$$
\mathcal{H}_{\gamma} \simeq L^{2}\left(G^{E}, \mathrm{~d} \mu_{H}^{\otimes E}\right)
$$

where $G=S U(2)$ is the gauge group acting on the fields $\left(A_{a}^{I}, E_{J}^{b}\right)$, and $\mathrm{d} \mu_{H}$ is the normalized Haar measure on $G$. Each of these $\mathcal{H}_{\gamma}$ is left invariant by the gauge transformations induced by the Gauss constraints $G_{I}(x)$. The restriction $\mathcal{G}_{\gamma}$ of the set of gauge transformations to $\mathcal{H}_{\gamma}$ is isomorphic to

$$
\mathcal{G}_{\gamma} \simeq G^{V}
$$

where $V$ is the number of vertices in the graph $\gamma$. The action of an element $\vec{k} \in G^{V}$ on a square-integrable function $\psi: G^{E} \rightarrow \mathbb{C}$ in $\mathcal{H}_{\gamma}$ is given by the following formula:

$$
\alpha_{\vec{k}} \psi\left(h_{e_{1}}, \ldots, h_{e_{E}}\right):=\psi\left(k_{b\left(e_{1}\right)} h_{e_{1}} k_{f\left(e_{1}\right)}^{-1}, \ldots, k_{b\left(e_{E}\right)} h_{e_{E}} k_{f\left(e_{E}\right)}^{-1}\right),
$$

where $b\left(e_{m}\right)$ and $f\left(e_{m}\right)$ are the beginning and end points of the edge $e_{m}$. So, the gauge transformations act only at the vertices of a graph.

In particular, one can write down the projector onto the gauge-invariant Hilbert space for functions in $\mathcal{H}_{\gamma}$ :

$$
\begin{aligned}
\mathcal{P} f\left(h_{e_{1}}, \ldots, h_{e_{E}}\right) & :=\int_{G^{V}} \mathrm{~d} \mu_{H}\left(k_{1}, \ldots, k_{V}\right) \alpha_{k_{1}, \ldots k_{V}} f\left(h_{e_{1}} \ldots, h_{e_{E}}\right) \\
& =\int_{G^{V}} \mathrm{~d} \mu_{H}\left(k_{1}, \ldots, k_{V}\right) f\left(k_{b\left(e_{1}\right)} h_{e_{1}} k_{f\left(e_{1}\right)}^{-1}, \ldots, k_{b\left(e_{E}\right)} h_{e_{E}} k_{f\left(e_{E}\right)}^{-1}\right) .
\end{aligned}
$$

Since $G^{V}$ is compact, the integral exists and defines a projector,

$$
\mathcal{P}: \mathcal{H}_{\gamma} \longrightarrow \mathcal{H}_{\gamma},
$$

onto a sub-Hilbert space of $\mathcal{H}_{\gamma}$. In particular, the gauge-invariant functions on a graph form a subset of all square-integrable functions on a graph. The gauge-invariant Hilbert spaces can be described using intertwiners between irreducible representations of $S U(2)$, and a basis for the gauge-invariant Hilbert spaces $\mathcal{P} \mathcal{H}_{\gamma}$ can be written down in terms of gauge-invariant spin network functions [9].

\section{Complexifier coherent states}

The complexifier coherent states (CCS) are states first constructed for quantum mechanics on arbitrary compact Lie groups $[10,11]$. They are natural generalizations of the harmonic oscillator coherent states (HOCS) for quantum mechanics on a real line, which are given by

$$
|z\rangle=\sum_{n=0}^{\infty} \frac{z^{n}}{\sqrt{n !}}|n\rangle .
$$

The HOCS can be seen as minimal uncertainty states, or states that correspond to the system of being in a quantum state close to a classical phase-space point $z=q+\mathrm{i} p$. 
Complexifier coherent states for quantum mechanics on a compact Lie group $G$ are given by a choice of a complexifier $\hat{C}$, a positive number $t>0$ and a point $g \in G^{\mathbb{C}}$. If one chooses the complexifier to be the negative Laplacian $-\Delta$ on $G$, then the states are given by

$$
\psi_{g}^{t}(h)=\sum_{\pi} \mathrm{e}^{-\lambda_{\pi} \frac{t}{2}} d_{\pi} \operatorname{tr} \pi\left(g h^{-1}\right),
$$

where the sum runs over all irreducible finite-dimensional representations $\pi$ of $G, \lambda_{\pi}$ is the eigenvalue of $-\Delta$ corresponding to the eigenfunction $h \rightarrow \operatorname{tr} \pi(h)$, and $d_{\pi}$ is the dimension of $\pi$. In the case of $G=S U(2)$, this reads

$$
\psi_{g}^{t}(h)=\sum_{j \in \frac{1}{2} \mathbb{N}} \mathrm{e}^{-j(j+1) \frac{t}{2}}(2 j+1) \operatorname{tr}_{j}\left(g^{-1} h\right),
$$

where $\operatorname{tr}_{j}$ is the trace in the spin- $j$ representation, and $g \in S U(2)^{\mathbb{C}}=S L(2, \mathbb{C})$. These states and their properties have been investigated in $[2,3]$. It could be shown that these states are sharply peaked around their labels $g \in S L(2, \mathbb{C})$, i.e. the overlap

$$
\mathrm{i}^{t}\left(g_{1}, g_{2}\right)=\frac{\left|\left\langle\psi_{g_{1}}^{t} \mid \psi_{g_{2}}^{t}\right\rangle\right|^{2}}{\left\|\psi_{g_{1}}^{t}\right\|^{2}\left\|\psi_{g_{2}}^{t}\right\|^{2}}
$$

equals 1 for $g_{1}=g_{2}$, but for $g_{1} \neq g_{2}$ goes to 0 faster than any power of $t$ as $t \rightarrow 0$, i.e. is $O\left(t^{\infty}\right)$. Furthermore, the $S U(2)$ CCS reproduce classical values of quantized phase space functions. For example, let $F: S L(2, \mathbb{C}) \rightarrow \mathbb{R}$ be a function on phase space, and $\hat{F}$ the corresponding quantized operator. Then

$$
\frac{\left\langle\psi_{g_{1}}^{t}|\hat{F}| \psi_{g_{2}}^{t}\right\rangle}{\left\langle\psi_{g_{1}}^{t} \mid \psi_{g_{2}}^{t}\right\rangle}=F\left(g_{2}\right)\left(1+f\left(g_{1}, g_{2}, t\right)\right),
$$

where $f$ is a function of $g_{1}, g_{2} \in S L(2, \mathbb{C})$ growing only polynomially in the complex directions, and is of order $O(t)$. This gives an immediate interpretation of the labeling parameter $g \in S L(2, \mathbb{C})$ : it corresponds to a point in the classical phase space, and (3.4), (3.5) show that $\psi_{g}^{t}$ defines a quantum state being close to the classical state $g$, with quantum fluctuations determined by $t$. In particular, one can see that the limit $t \rightarrow 0$ corresponds to the semiclassical limit of the theory, being classical mechanics on $S U(2)$.

The CCS can be used in the LQG framework. The classical phase space of GR is the space of Cauchy data $\left(A_{a}^{I}, E_{J}^{b}\right)$, i.e. the Ashtekar connection and the canonically conjugated electric field on the spatial slice $\Sigma(2.1)$. The easiest example of a complexifier can be constructed by the following method: choose a finite graph $\gamma=\left\{e_{1}, \ldots, e_{E}\right\}$ with $E$ edges and a dual polyhedronal decomposition $\gamma^{*}=\left\{S_{1}, \ldots, S_{E}\right\}$ of $\Sigma$, i.e. a collection of $E$ non-intersecting surfaces such that $e_{k} \cap S_{l}$ consists of exactly one point if $k=l$, and is empty otherwise. Then define the following functions of phase space:

$$
\begin{aligned}
& h_{k}\left(A_{a}^{I}\right)=\mathcal{P} \exp \int_{e_{k}} A \in S U(2) \\
& P_{k}\left(A_{a}^{I}, E_{J}^{b}\right)=\frac{1}{8 \pi G \beta} \int_{S_{k}} * \tilde{E} \in \mathfrak{s u}(2) .
\end{aligned}
$$

Here $\mathcal{P}$ is the path-ordered exponential, $\tilde{E}$ is defined by parallely transporting the electric field $E_{J}^{b}$ to the beginning point of $e_{k}$ (details can be found in [2]). * denotes the Hodge dual, which makes $* \tilde{E}$ into an $\mathfrak{s u}(2)$-valued 2-form, which can hence be integrated over $S_{k}$. Note that, due to the parallel transport, $P_{k}$ depends on both $A_{a}^{I}$ and $E_{J}^{b}$.

The functions $h_{k}$ and $P_{k}, k=1, \ldots, E$ are phase-space functions that serve as coordinates on a finite-dimensional submanifold of the infinite-dimensional phase space of GR. 
By the identification

$$
\mathrm{e}^{P_{k}\left(A_{a}^{I}, E_{J}^{b}\right)} h_{k}\left(A_{a}^{I}\right)=g_{k}\left(A_{a}^{I}, E_{J}^{b}\right) \in S L(2, \mathbb{C}),
$$

this submanifold can be identified with $S L(2, \mathbb{C})^{E}$, where the configuration space is given by $S U(2)^{E}$. This allows the use of the complexifier coherent states for quantum mechanics on $S U(2)$, and results in the states

$$
\psi_{g_{1}, \ldots, g_{E}}^{t}\left(h_{1}, \ldots, h_{E}\right)=\prod_{k=1}^{E} \psi_{g_{k}}^{t}\left(h_{k}\right) .
$$

The connection of the labels $g_{k} \in S L(2, \mathbb{C})$ with the phase space is given by (3.7): in the polar decomposition $g_{k}=H_{k} u_{k}$ into Hermitean $H_{k}$ and unitary $u_{k}$, the holonomy of the Ashtekar connection along $e_{k}$ is given by $u_{k}$, and $H_{k}$ is connected to (the exponential of) the electric field integrated over $S_{k}$.

The semiclassicality parameter $t$ here is given by the comparison of the Planck length $\ell_{P}$ to a classical length scale $a$ [2]:

$$
t=\frac{\ell_{P}^{2}}{a^{2}}
$$

By choosing a scale $a$ to be the characteristic length in a situation under consideration, one can arrive at different $t$. For classical physics, i.e. $a=1 \mathrm{~cm}$, one gets $t \approx 10^{-70}$. The smaller $t$, the more the quantum fluctuations are suppressed, which is in accordance with the interpretation of $t$ as a semiclassicality parameter.

The states (3.8) are special cases for the semiclassical states employed in LQG. Generalized forms correspond to different complexifiers $\hat{C}$ on $\mathcal{H}_{\text {kin }}$, which are not connected to the Laplacian, but, e.g., to the volume operator $\hat{V}$, have different semiclassicality parameters $t_{k}>0$ for different edges $e_{k}{ }^{3}$ or are superpositions of states (3.8) over different graphs. Details can be found in $[12,13]$.

\subsection{Geometric version of the inner product}

In the following we will give a geometric interpretation of the inner product of the $S U(2)$ CCS. This has already been done for their $U(1)$ counterparts in [1]. We start from the form of the CCS (3.2), from which the inner product of two CCS can be computed:

$$
\left\langle\psi_{g}^{t} \mid \psi_{g^{\prime}}^{t}\right\rangle=\sum_{j \in \frac{1}{2} \mathbb{N}} \mathrm{e}^{-j(j+1) t}(2 j+1) \operatorname{tr}_{j}\left(g^{\dagger} g^{\prime}\right) .
$$

In [2], an application of the Poisson summation formula was used to bring (3.10) into the form

$$
\left\langle\psi_{g}^{t} \mid \psi_{g^{\prime}}^{t}\right\rangle=\frac{2 \mathrm{e}^{\frac{t}{4}}}{\pi} \sqrt{\frac{\pi}{t}} \sum_{n \in \mathbb{Z}} \frac{z-2 \pi n}{\sin (z-2 \pi n)} \exp \left[-\frac{(z-2 \pi n)^{2}}{t}\right],
$$

where

$$
\cos z=\frac{1}{2} \operatorname{tr}\left(g^{\dagger} g^{\prime}\right)
$$

Note that by (3.12) $z$ is only defined up to a sign and a shift $z+2 \pi n$ for some $n \in \mathbb{Z}$. But (3.11) has exactly the corresponding symmetries, such that the formula is well defined. In [3], form

3 We are just considering the simple case of constant $t$ here, noting that qualitative results carry over to the case of, e.g., edge-dependent semiclassicality parameters $t_{k}$, which should be of physical interest for situations in which the quantum fluctuations of geometry vary, e.g. if there are spacetime regions with large quantum fluctuations embedded within nearly classical spacetime regions. 
(3.11) was chosen for convenience. In the limit $t \rightarrow 0$ one can, if one chooses $\operatorname{Re} z \in(-\pi, \pi)$, neglect all the terms with $n \neq 0$ in (3.11), since they are exponentially damped compared to the $n=0$ term. This simplified calculations immensely. We will use the same form, partly for the same reasons, but also in order to show how the inner product between the CCS on $S U(2)$ can be interpreted via the geometry on its complexification $S U(2)^{\mathbb{C}}=S L(2, \mathbb{C})$. In order to do this, we have to talk about geodesics on $S L(2, \mathbb{C})$.

\subsection{Geodesics on $S L(2, \mathbb{C})$}

The exponential map

$$
\mathbb{C}^{3} \ni \vec{z} \longmapsto \mathrm{e}^{\mathrm{i} \vec{z} \cdot \vec{\sigma}}=\cos z+\mathrm{i} \frac{\sin z}{z} \vec{z} \cdot \vec{\sigma} \in S L(2, \mathbb{C}),
$$

where $\sigma_{I}, I=1,2,3$ are the Pauli spin matrices and $\vec{z} \in \mathbb{C}^{3}$, such that $\mathrm{i} \vec{z} \cdot \vec{\sigma} \in \mathfrak{s l}_{2} \mathbb{C}$. Furthermore $z:=\sqrt{z_{1}^{2}+z_{2}^{2}+z_{3}^{2}}$ is determined up to a sign, but the functions in (3.13) are symmetric in $z$, so everything is well defined.

This does not only define a chart that covers all of $S L(2, \mathbb{C})$, but allows one also to write down the geodesics immediately. To do so, we first note that the group structure on $S L(2, \mathbb{C})$ determines a (pseudo-)metric. On its Lie algebra $\mathfrak{s l}_{2} \mathbb{C}$, the Killing form,

$$
(A, B) \longmapsto-\frac{1}{2} \operatorname{tr}(A B)
$$

can be defined and is a bilinear non-degenerate form on $\mathfrak{s l}_{2} \mathbb{C}=T_{\mathbb{1}} S L(2, \mathbb{C})$. Since $S L(2, \mathbb{C})$ is a group, one can pull back (3.14) to every other tangent space on $S L(2, \mathbb{C})$ by right (or, which gives the same result, left) multiplication. This defines a non-degenerate, bi-invariant pseudo-Riemannian metric called the Killing metric, which is given in coordinates by

$$
h_{I J}:=\frac{1}{2} \operatorname{tr}\left(\left(g^{-1} \partial_{I} g\right)\left(g^{-1} \partial_{J} g\right)\right) \text {. }
$$

From this a connection can be formed, and geodesics can be defined. In particular, the geodesics going through $\mathbb{1} \in S L(2, \mathbb{C})$ are given by

$$
t \longmapsto \mathrm{e}^{\mathrm{i} t z_{I} \sigma^{I}}
$$

where $\vec{z} \in \mathbb{C}^{3}$, such that $\overrightarrow{i z} \cdot \vec{\sigma} \in \mathfrak{s l}_{2} \mathbb{C}$ is the velocity vector at $t=0$. An immediate consequence of the bi-invariance of the Killing metric is that, given a geodesic $t \rightarrow \gamma(t)$ on $S L(2, \mathbb{C})$, for any $g_{1}, g_{2} \in S L(2, \mathbb{C})$, also $t \rightarrow g_{1} \gamma(t) g_{2}$ is a geodesic, which allows us to compute all geodesics from $g_{1}$ to $g_{2}$ by computing all from $\mathbb{1}$ to $g=g_{1}^{-1} g_{2}$. In general, there will be more than one, and from (3.16) one can see that any geodesic from $\mathbb{1}$ to $g=\exp \vec{i} \cdot \vec{\sigma}$ is given by

$$
[0,1] \ni t \longmapsto \mathrm{e}^{\mathrm{i} t \vec{z} \cdot \vec{\sigma}}
$$

Thus, different geodesics arise from the fact that $\exp \vec{i} \cdot \vec{\sigma}=\exp i \vec{w} \cdot \vec{\sigma}$ for $\vec{z} \neq \vec{w}$, i.e. $\vec{z} \in \mathbb{C}^{3}$ is not uniquely determined by $g \in S L(2, \mathbb{C})$ We will classify the different possible cases as follows.

Lemma 3.1. Let $\vec{z} \in \mathbb{C}^{3}$. Then $z=\sqrt{\vec{z} \cdot \vec{z}}$ is determined only up to a sign. Define $g=\exp \vec{i} \cdot \vec{\sigma}$, then exactly one of the following is true:

- Case 1: $z=0$ and $\vec{z} \neq 0$.

- Case 2: $z=2 \pi n$ for $n \in Z \backslash\{0\}$ or $\vec{z}=0$.

- Case 3: $z=\pi+2 \pi n$ for $n \in Z$.

- Case 4: $z \notin 2 \pi \mathbb{Z}$ and $z \notin \pi+2 \pi \mathbb{Z}$.

6 
In case 1, there is exactly one geodesic from 11 to $g$, in cases 2 and 3 there are uncountably many, and in case 4 there are countably many geodesics from $\mathbb{1}$ to $g$.

Proof. In the first case, where $z_{1}^{2}+z_{2}^{2}+z_{3}^{2}=0$, we have

$$
g=\exp \vec{i} \cdot \vec{\sigma}=\mathbb{1}+\mathrm{i} \vec{z} \cdot \vec{\sigma} .
$$

Let now $\vec{w} \in \mathbb{C}^{3}$ with exp $\mathrm{i} \vec{w} \cdot \sigma=g$. Then it follows from (3.13) that $\cos w=1$. In particular, $w=2 \pi n$ for some $n \in \mathbb{Z}$. But if $n \neq 0$, then $\sin w / w=0$ and $g=\mathbb{1}$; hence $\vec{z}=0$, which is excluded in this case. So $n=0$ and hence $\mathrm{i} \vec{w} \cdot \vec{\sigma}=\mathrm{i} \vec{z} \cdot \vec{\sigma}$, so $\vec{z}=\vec{w}$. In particular, the vector $\vec{z}$ is unique. Hence, there is only one geodesic from $\mathbb{1}$ to $g$.

The second and third cases can readily be seen to correspond to $g=\mathbb{1}$ and $g=-\mathbb{1}$. Also, it can easily be seen that all geodesics from $\mathbb{1}$ to itself are given by

$$
[0,1] \ni t \longmapsto \mathrm{e}^{2 \pi n t \vec{\phi} \cdot \vec{\sigma}}
$$

for arbitrary $n \in \mathbb{Z}$ and $\vec{\phi} \in S^{2}$, i.e. $\|\vec{\phi}\|=1$. Similarly, all geodesics from $\mathbb{1}$ to $-\mathbb{1}$ are given by

$$
[0,1] \ni t \longmapsto \mathrm{e}^{(2 \pi n+\pi) t \vec{\phi} \cdot \vec{\sigma}} .
$$

So, there are uncountably many geodesics in both cases.

Case 4 is the generic case. Let $\vec{w}, \vec{z} \in \mathbb{C}^{3}$ such that $\mathrm{e}^{\mathrm{i} z \cdot \vec{\sigma}}=\mathrm{e}^{\mathrm{i} \vec{w} \cdot \vec{\sigma}}$. Thus, by (3.13) we have $\cos w=\cos z$, so

$$
w^{2}=(z+2 \pi n)^{2}
$$

for some $n \in \mathbb{Z}$. Furthermore, from the linear independence of the $\sigma$-matrices, it follows that

$$
\vec{w}=\frac{z+2 \pi n}{z} \vec{z} .
$$

Thus we see that, if one chooses $\vec{z} \in \mathbb{C}^{3}$ such that $g=\exp \vec{i} \cdot \vec{\sigma}$, then all other $\vec{w}$ with $g=\operatorname{expi} \vec{w} \cdot \vec{\sigma}$ can be obtained by (3.22), via letting $n$ go through $\mathbb{Z}$. Thus, there are countably many geodesics from $\mathbb{1}$ to $g$.

This finishes the proof.

Knowing this, we can turn to defining the complex length of a geodesic, or more generally of an $h$-regular curve. Let $t \rightarrow \gamma(t)$ be an $h$-regular curve. That is, $\gamma$ does not necessarily have to be a geodesic, but the Killing form $h$ shall never annihilate the velocity vector, i.e. $h(\dot{\gamma}, \dot{\gamma}) \neq 0$ along the curve. Then, with the help of the Killing metric, the complex length of $\gamma$ can be defined via

$$
l(\gamma):=\int_{\gamma} \sqrt{h(\dot{\gamma}, \dot{\gamma})} .
$$

Note that this is in complete analogy to the definition of the length of a curve with the help of a Riemannian metric, the only difference being a sign issue. Since generically $h(\dot{\gamma}, \dot{\gamma})$ will be complex on the path, its square root is determined up to a sign, but since the integrand never vanishes, this sign can be chosen such that $\sqrt{h(\dot{\gamma}, \dot{\gamma})}$ is smooth along the curve, and there are exactly two such choices. Thus, $l(\gamma)$ is only defined up to a sign.

Now, to compute the complex length of a geodesic (3.20) from $\mathbb{1}$ to $g=\exp i \vec{z} \cdot \sigma$ for $\vec{z} \in \mathbb{C}^{3}$ is easy. Since the velocity vector is parallely transported along geodesics, the integrand in (3.23) is constant along $[0,1]$. In particular, it equals its value at $t=0$, which is nothing but the Killing form on $\mathfrak{s l}_{2} \mathbb{C}$, i.e.

$$
l(\gamma)=\sqrt{\vec{z} \cdot \vec{z}}=z .
$$


Note that there are also geodesics $t \rightarrow \gamma(t)$ where $\dot{\gamma}(t)$ vanishes. But since the velocity vector of a geodesic is parallely transported, $\dot{\gamma}$ vanishes identically for these curves, and thus we can consistently define the complex length of such a geodesic to be 0 .

Knowing this, we arrive at the main part of this section.

Lemma 3.2. Let $g_{1}, g_{2} \in S L(2, \mathbb{C})$ and

$$
j^{t}\left(g_{1}, g_{2}\right)=\left\langle\psi_{g_{1}}^{t} \mid \psi_{g_{2}}^{t}\right\rangle
$$

be the inner product between complexifier coherent states. Apart from the cases $g_{1}^{c}=g_{2}$ or $g_{1}^{c}=-g_{2}$, when there are uncountably many geodesics between $g_{1}^{c}$ and $g_{2}$, and $g_{1}^{\dagger} g_{2}=\operatorname{expi} \vec{z} \cdot \vec{\sigma}$ with $\vec{z} \cdot \vec{z}=0$, when there is only one geodesic between $g_{1}^{c}$ and $g_{2}$, the inner product is given by

$$
j^{t}\left(g_{1}, g_{2}\right)=\frac{2 \mathrm{e}^{\frac{t}{4}}}{\pi} \sqrt{\frac{\pi}{t}} \sum_{\substack{\gamma \text { geodesic } \\ \text { from } g_{1}^{c} \text { to } g_{2}}} \frac{l(\gamma)}{\sin l(\gamma)} \mathrm{e}^{-\frac{l(\gamma)^{2}}{t}} .
$$

Proof. Write $g=g_{1}^{\dagger} g_{2}$. Since neither $g_{1}^{c}=g_{2}$ nor $g_{1}^{c}=-g_{2}$, it follows that neither $g=\mathbb{1}$ nor $g=-\mathbb{1}$. Also, there is more than one $\vec{z}$ with $\exp \vec{i} \cdot \vec{\sigma}=g$. Then we know by lemma 3.1 that there are countable infinitely many geodesics from $g_{1}^{c}$ to $g_{2}$, or equivalently from $\mathbb{1}$ to $g=g_{1}^{\dagger} g_{2}$. Choose a $\vec{z} \in \mathbb{C}^{3}$ such that $g=\operatorname{expi} \vec{z} \cdot \vec{\sigma}$. We have already seen that this amounts to choosing a geodesic from $\mathbb{1}$ to $g$. Then $z=\sqrt{\vec{z} \cdot \vec{z}}$ is the complex length of this geodesic, which is determined up to a sign. By (3.21) we see that all other complex lengths are determined by letting $n$ run through $\mathbb{Z}$, which tells us that

$$
\begin{aligned}
j^{t}\left(g_{1}, g_{2}\right) & =\frac{2 \mathrm{e}^{\frac{t}{4}}}{\pi} \sqrt{\frac{\pi}{t}} \sum_{n \in \mathbb{Z}}^{3} \frac{z-2 \pi n}{\sin (z-2 \pi n)} \exp \left[-\frac{(z-2 \pi n)^{2}}{t}\right] \\
& =\frac{2 \mathrm{e}^{\frac{t}{4}}}{\pi} \sqrt{\frac{\pi}{t}} \sum_{\substack{\gamma \text { geodesic } \\
\text { from } g_{1}^{c} \text { to } g_{2}}} \frac{l(\gamma)}{\sin l(\gamma)} \mathrm{e}^{-\frac{l(\gamma)^{2}}{t}},
\end{aligned}
$$

which proves the lemma.

The inner product between the two coherent states is thus a function intimately related to the geometry on $S L(2, \mathbb{C})$. Unfortunately, formula (3.26) is valid only if there are countably infinitely many geodesics from $g_{1}^{c}$ to $g_{2}$. On the other hand, the pairs $\left(g_{1}, g_{2}\right)$ that do not satisfy this condition (i.e. that either have $g=g_{1}^{\dagger} g_{2}= \pm \mathbb{1}$ or $g=\exp \vec{i} \cdot \vec{\sigma}$ with a nonzero $\vec{z} \in \mathbb{C}^{3}$ that has $\left.z^{2}=\vec{z} \cdot \vec{z}=0\right)$ are a subset of measure zero in $\operatorname{SL}(2, \mathbb{C}) \times S L(2, C)$.

The conjugation $g \rightarrow g^{c}$ can, in the polar decomposition of $g=H u$ into Hermitean $H$ and unitary $u$, be written as

$$
H u \longmapsto H^{-1} u \text {. }
$$

For the norm of a complexifier coherent state we thus get

$$
\begin{aligned}
\left(\frac{2 \mathrm{e}^{\frac{t}{4}}}{\pi} \sqrt{\frac{\pi}{t}}\right)^{3}\left\|\psi_{g}^{t}\right\|^{2} & =\sum_{\substack{\gamma \text { geodesic } \\
\text { from } H^{-1} u \text { to } H u}} \frac{l(\gamma)}{\sin l(\gamma)} \mathrm{e}^{-\frac{l(\gamma)^{2}}{t}}=\sum_{\substack{\gamma \text { geodesic } \\
\text { from } 1 \text { to } H^{2}}} \frac{l(\gamma)}{\sin l(\gamma)} \mathrm{e}^{-\frac{l(\gamma)^{2}}{t}} \\
& =\sum_{n \in \mathbb{Z}} \frac{l+2 \pi \text { in }}{\sinh (l+2 \pi \text { in })} \mathrm{e}^{\frac{(l+2 \pi \text { in })^{2}}{t}}=\mathrm{e}^{\frac{l^{2}}{t}}\left(1+O\left(t^{\infty}\right)\right),
\end{aligned}
$$


with $l=|\vec{l}|, \vec{l} \in \mathbb{R}^{3}$ and $H=\mathrm{e}^{l_{I} \sigma^{I}}$. For two complexifier coherent states peaked on elements $g \in S U(2) \subset S L(2, \mathbb{C})$, we have $\vec{l}=0$, hence in this case

$$
\left\|\psi_{g}^{t}\right\|=\sqrt{\frac{2 \mathrm{e}^{\frac{t}{4}}}{\pi} \sqrt{\frac{\pi}{t}}}\left(1+O\left(t^{\infty}\right)\right)
$$

Note that the complex length (3.23) of a curve $\gamma$ lying entirely in $S U(2) \subset S L(2, \mathbb{C})$ is real, and its square $l(\gamma)^{2}$ coincides with the geodesic distance $\mathrm{d}(\gamma)^{2}$ on $S U(2)$ determined by the Killing metric. Thus, the overlap of two complexifier coherent states peaked on elements $g_{1}, g_{2} \in S U(2) \subset S L(2, \mathbb{C})$ is given by

$$
\frac{\left\langle\psi_{g_{1}}^{t} \mid \psi_{g_{2}}^{t}\right\rangle}{\left\|\psi_{g_{1}}^{t}\right\|\left\|\psi_{g_{2}}^{t}\right\|}=\sum_{\substack{\gamma \text { geodesic } \\ \text { from } g_{1} \text { to } g_{2}}} \frac{d(\gamma)}{\sin d(\gamma)} \mathrm{e}^{-\frac{d(\gamma)^{2}}{t}}\left(1+O\left(t^{\infty}\right)\right)
$$

since $g^{c}=g$ for $g \in S U(2)$.

For states labeled by elements $g_{1}, g_{2} \in S U(2)$, this immediately shows the nice peakedness properties these states have. For two states being labeled by different elements, the overlap is, basically, a sum over terms being proportional to Gaussians in the lengths of geodesics from one to the other elements. For small $t>0$, the term with the shortest distance dominates all other terms, and the overlap is nearly proportional to a Gaussian in the geodesic distance on $S U(2)$.

\section{Gauge-invariant coherent states for $G=S U(2)$}

In the following, we will construct the gauge-invariant coherent states on a graph by projecting the complexifier coherent states on the gauge-invariant subspace. This will result in states labeled by gauge-equivalence classes of phase-space points which will be identified with points in gauge-invariant phase space. Afterwards, we will examine these states for some particular graphs. There we will show that gauge-invariant coherent states labeled by points in gauge-invariant phase space have an overlap that vanishes exponentially as the two points become distinct. This will demonstrate the peakedness properties for these states, which make these states useful for semiclassical analysis in the gauge-invariant sector.

It should be noted that in the spin foam approach of quantum gravity one makes frequent use of states that have been introduced by Livine and Speziale (see, e.g., [14]) that are gauge invariant and also called coherent states. These are states that come from a Perelomov-type approach, rather than a complexifier. Another apparent difference between the Livine-Speziale states and those considered in this paper is the fact that their states have zero uncertainty in the fluxes, while being maximally spread with respect to the holonomies, while the states presented here will have a finite spread in both variables. In this sense the Livine-Speziale states do not correspond to a point in classical phase space, while their much simpler form makes them excellent tools for handling expressions such as the spin foam vertices in the Freidel-Krasnov model [15]. A comparison to a plane wave, rather than a finite Gaussian wave package suggests itself, although very much like the wave packets, the Livine-Speziale states form an overcomplete set rather than an orthonormal basis. A closer comparison between these states and those presented here would be desirable. 


\subsection{Gauge-invariant functions}

The Hilbert space $\mathcal{H}_{\gamma}$ consists of functions on $S U(2)^{E}$, square integrable with respect to the $E$-fold Haar measure $\mathrm{d} \mu_{H}^{\otimes E}$ :

$$
H_{\gamma} \simeq L^{2}\left(G^{E}, \mathrm{~d} \mu_{H}^{\otimes E}\right)
$$

In section 2, the gauge action of the Gauss constraints on the kinematical Hilbert space has been discussed, in particular the gauge transformation of a function cylindrical on a graph (2.5). Denote by $\pi: S U(2)^{E} \rightarrow S U(2)^{E} / S U(2)^{V}$ the projection map of $S U(2)^{E}$ to the space of orbits under the action

$$
\begin{aligned}
& \alpha: S U(2)^{V} \times \mathcal{H}_{\gamma} \longrightarrow \mathcal{H}_{\gamma} \\
& \left(\alpha_{k_{v_{1}}, \ldots, k_{v_{V}}} \psi\right)\left(h_{e_{1}}, \ldots, h_{e_{E}}\right)=\psi\left(k_{b\left(e_{1}\right)}^{-1} h_{e_{1}} k_{f\left(e_{1}\right)}, \ldots, k_{b\left(e_{E}\right)}^{-1} h_{e_{E}} k_{f\left(e_{E}\right)}\right)
\end{aligned}
$$

of the gauge group $S U(2)^{V}$. Then

$$
\mathcal{P} \mathcal{H}_{\gamma} \simeq L^{2}\left(S U(2)^{E} / S U(2)^{V}, \pi_{*} \mathrm{~d} \mu_{H}^{\otimes E}\right)
$$

So, the orbifold $S U(2)^{E} / S U(2)^{V}$ is treated as gauge-invariant configuration space. This space can be formulated nicely in terms of cohomology, in particular

$$
S U(2)^{E} / S U(2)^{V} \simeq H^{1}(\gamma, S U(2)),
$$

where $H^{1}(\gamma, S U(2))$ is the first Čech-cohomology group on the graph $\gamma$ with values in $S U(2)$.

\subsection{Gauge-invariant coherent states and gauge orbits}

In the following sections, we will describe the projections of the complexifier coherent states (CCS),

$\psi_{g_{1}, \ldots, g_{E}}^{t}\left(h_{1}, \ldots, h_{E}\right)=\prod_{e \in E(\gamma)} \sum_{j_{e} \in \frac{1}{2} \mathbb{N}} \mathrm{e}^{-j_{e}\left(j_{e}+1\right) \frac{t}{2}}\left(2 j_{e}+1\right) \operatorname{tr}_{j_{e}}\left(g_{e} h_{e}^{-1}\right)$,

onto the gauge-invariant subspace $\mathcal{P} \mathcal{H}_{\gamma} \subset \mathcal{H}_{\gamma}$. This will define the gauge-invariant coherent states, and we will investigate some of their properties. Since the gauge integral will be too complicated to perform exactly, we will have to rely on numerical investigations in some cases, where analytical methods are not enough.

By the explicit form (4.4), the action (4.1) also induces an action on the set of coherent states, hence an action on $S L(2, \mathbb{C})^{E}$ via

$$
\alpha_{\vec{k}} \psi_{\vec{g}}^{t}=\alpha_{k_{v_{1}}, \ldots, k_{v_{V}}} \psi_{g_{e_{1}}, \ldots, g_{e_{E}}}^{t}=\psi_{k_{f\left(e_{1}\right)}^{t} g_{e_{1}} k_{b\left(e_{1}\right)}^{-1}, \ldots, k_{f\left(e_{E}\right)} g_{e_{E}} k_{b\left(e_{E}\right)}^{-1}}^{t}=: \psi_{\alpha_{\vec{k}} \vec{g}}^{t}
$$

The gauge-invariant coherent states are the image of the complexifier coherent states (4.4) under the action of $\mathcal{P}(2.6)$ :

$$
\Psi_{[\vec{g}]}^{t}:=\mathcal{P} \psi_{\vec{g}}^{t}=\int_{S U(2)^{V}} \mathrm{~d} \mu_{H}(\vec{k}) \psi_{\alpha_{\vec{k}} \vec{g}}^{t} .
$$

Naively, one could think that the labeling of the gauge-invariant coherent state is now $[\vec{g}] \in\left\{\alpha_{\vec{k}} \vec{g}, \vec{k}=\left(k_{v_{1}}, \ldots, k_{v_{V}}\right) \in S U(2)^{V}\right\}$. However, this is not the case! $\mathcal{P}$ can map states labeled by $\vec{g}_{1}, \vec{g}_{2} \in S L(2, \mathbb{C})^{E}$ to the same state in $\mathcal{P} \mathcal{H}_{\gamma}$ although there is no $\vec{k} \in S U(2)^{V}$ such that $\alpha_{\vec{k}} \vec{g}_{1}=\vec{g}_{2}$. The reason for this is the holomorphic dependence of the CCS on their labeling parameter. To shed some light on this issue, we need the following lemma. 
Lemma 4.1. Let $G$ be a compact Lie group and $G^{\mathbb{C}}$ its complexification. Let $f: G^{\mathbb{C}} \rightarrow \mathbb{C}$ be an analytic function (i.e. holomorphic w.r.t to the complex structure on $G^{\mathbb{C}}$. Then, for any $g, g^{\prime} \in G^{\mathbb{C}}$, we have

$$
\int_{G} \mathrm{~d} \mu_{H}(h) f(h)=\int_{G} \mathrm{~d} \mu_{H}(h) f\left(g h g^{\prime}\right) .
$$

Proof. Since $f$ is analytic on $G^{\mathbb{C}}$, it is in particular continuous on $G$, which is compact. The restriction of $f$ on $G$ and hence $|\tilde{f}|^{2}$ are uniformly bounded functions on $G$. So the restriction is square integrable, and by the Peter-Weyl theorem it can be decomposed into its Fourier coefficients:

$$
f(h)=\sum_{\pi} \sum_{m, n} \sqrt{d_{\pi}} c_{\pi m n} \pi(h)_{m n},
$$

for all $h \in G$. The functions $h \rightarrow \pi(h)_{m n}$ are all anayltic, and is the matrix element function of an irreducible representation of $G^{\mathbb{C}}$. Then, for any $g, g^{\prime}$

$$
\begin{aligned}
\int_{G} \mathrm{~d} \mu_{H}(h) f\left(g h g^{\prime}\right) & =\sum_{\pi} \sum_{m, n} \sqrt{d_{\pi}} c_{\pi m n} \int_{G} \mathrm{~d} \mu_{H}(h) \pi\left(g h g^{\prime}\right)_{m n} \\
& =\sum_{\pi} \sum_{m, m^{\prime}, n^{\prime}, n} \sqrt{d_{\pi}} \pi(g)_{m m^{\prime}} c_{\pi m n} \pi\left(g^{\prime}\right)_{n^{\prime} n} \int_{G} \mathrm{~d} \mu_{H}(h) \pi(h)_{m n} .
\end{aligned}
$$

But the integral only gives a contribution for the trivial representation $\pi_{0}$, which is one dimensional, the corresponding Fourier coefficient corresponding to the integral of $f$ over $G$. So the only term remaining is

$$
\int_{G} \mathrm{~d} \mu_{H}(h) f\left(g h g^{\prime}\right)=\pi_{0}(g) c_{0} \pi_{0}\left(g^{\prime}\right)=c_{0}=\int_{G} \mathrm{~d} \mu(h) f(h) .
$$

This completes the proof.

Of course, the above lemma carries over to integrals over the $V$-fold product $S U(2)^{V}$. The gauge action $\alpha_{\vec{k}}$ of $S U(2)^{V}$ on the label set $S L(2, \mathbb{C})^{E}$,

$$
\alpha_{k_{v_{1}}, \ldots, k_{v_{V}}}\left(g_{e_{1}}, \ldots, g_{e_{E}}\right)=\left(k_{f\left(e_{1}\right)} g_{e_{1}} k_{b\left(e_{1}\right)}^{-1}, \ldots, k_{f\left(e_{E}\right)} g_{e_{E}} k_{b\left(e_{E}\right)}^{-1}\right),
$$

can obviously be continued analytically to an action of $S L(2, \mathbb{C})^{V}$ on $S L(2, \mathbb{C})^{E}$ simply by taking the same formula (4.7), but allowing for $\vec{k} \in S L(2, \mathbb{C})^{V}$. Let $\vec{g}_{1}, \vec{g}_{2} \in S L(2, \mathbb{C})^{E}$ such that they can be related by such a gauge transformation with elements $\vec{l} \in S L(2, \mathbb{C})^{V}$ :

$$
\vec{g}_{1} \in S L(2, \mathbb{C})^{E}, \quad \vec{g}_{2}=\alpha_{\vec{l}} \vec{g}_{1}, \quad \vec{l} \in S L(2, \mathbb{C})^{V} .
$$

Then, since the coherent states (4.4) depend analytically on their labels, i.e. for each $h \in S U(2)$ the function

$$
S L(2, \mathbb{C}) \ni g \longmapsto \psi_{g}^{t}(h) \in \mathbb{C}
$$

is analytic, the gauge integrand is also analytic. In particular, by (4.1), for each $\vec{h} \in S U(2)^{E}$ and $\vec{g} \in S L(2, \mathbb{C})^{E}$, the function,

$$
S U(2)^{V} \ni \vec{k} \longmapsto \psi_{\alpha_{\vec{k}} \vec{g}}^{t}(\vec{h}) \in \mathbb{C},
$$

is analytic, and can in an obvious way be extended to an analytic function on $S L(2, \mathbb{C})^{V}$. Hence, by lemma 4.1, we get

$$
\begin{aligned}
\mathcal{P} \psi_{\vec{g}_{1}}^{t} & =\int_{S U(2)^{V}} \mathrm{~d} \mu_{H}(\vec{k}) \psi_{\alpha_{\vec{k}} \vec{g}_{1}}^{t} \\
& =\int_{S U(2)^{V}} \mathrm{~d} \mu_{H}(\vec{k}) \psi_{\alpha_{\vec{k}} \alpha_{l} \vec{g}_{1}}^{t}=\int_{S U(2)^{V}} \mathrm{~d} \mu_{H}(\vec{k}) \psi_{\alpha_{\vec{k}} \vec{g}_{2}}^{t}=\mathcal{P} \psi_{\vec{g}_{2}}^{t},
\end{aligned}
$$

where the shift of integration variables $k_{v} \rightarrow k_{v} l_{v}$ has been used. 
The gauge-invariant coherent states are thus labeled by a semiclassicality parameter $t>0$ and an equivalence class $[\vec{g}]$ which is given by

$$
[\vec{g}]:=\left\{\alpha_{\vec{k}} \vec{g}, \vec{k}=\left(k_{v_{1}}, \ldots, k_{v_{V}}\right) \in S L(2, \mathbb{C})^{V}\right\} .
$$

Note that, at a second glance, it is quite natural that the gauge-invariant states are labeled by orbits of the complexified gauge action on $S L(2, \mathbb{C})^{E}$, i.e. on the orbifold $S L(2, \mathbb{C})^{E} / S L(2, \mathbb{C})^{V}$ rather than $S L(2, \mathbb{C})^{E} / S U(2)^{V}$, for dimensional reasons. The complexifier coherent states $\psi_{\vec{g}}^{t}$ are functions on $S U(2)^{E}$, which can be seen as the configuration space, while the states are labeled by elements in $S L(2, \mathbb{C})^{E}$, a space which has twice the number of real dimensions than $S U(2)^{E}$. In particular, since $S L(2, \mathbb{C})^{E}$ is diffeomorphic to the tangent bundle of $S U(2)^{E}$, it can be identified with the phase space of a system whose configuration space is $S U(2)^{E}$.

The gauge-invariant coherent states are, as we have seen in section 4.1, functions on the set $S U(2)^{E} / S U(2)^{V}$ of gauge orbits of $S U(2)^{E}$ under the gauge action $S U(2)^{V}$. This is not a manifold any more, since it contains singular points. So, it is not clear what its tangent bundle might be. However, the set $S L(2, \mathbb{C})^{E} / S L(2, \mathbb{C})^{V}$ of orbits of the tangent bundle $S L(2, \mathbb{C})^{E}$ under the complexified gauge action of $S L(2, \mathbb{C})^{V}$ serves as a natural candidate. It is an orbifold of twice the (real) dimension than $S U(2)^{E} / S U(2)^{V}$. This is not the case for $S L(2, \mathbb{C})^{E} / S U(2)^{V}$, as one can readily see. So, if we view $S U(2)^{E} / S U(2)^{V}$ as the gauge-invariant configuration space, then $S L(2, \mathbb{C})^{E} / S L(2, \mathbb{C})^{V}$ is the natural candidate for its gauge-invariant phase space.

\subsection{On semiclassical properties}

We are interested in the peakedness properties of the gauge-invariant coherent states, in particular the inner product

$$
J^{t}\left(\left[\vec{g}_{1}\right],\left[\vec{g}_{2}\right]\right):=\left\langle\Psi_{\left[\vec{g}_{1}\right]}^{t} \mid \Psi_{\left[\vec{g}_{2}\right]}^{t}\right\rangle,
$$

as well as their overlap

$$
I^{t}\left(\left[\vec{g}_{1}\right],\left[\vec{g}_{2}\right]\right):=\frac{\mid\left\langle\Psi_{\left[\vec{g}_{1}\right]}^{t}\left|\Psi_{\left[\vec{g}_{2}\right]}^{t}\right|^{2}\right.}{\left\|\Psi_{\left[\vec{g}_{1}\right]}^{t}\right\|^{2}\left\|\Psi_{\left[\vec{g}_{2}\right]}^{t}\right\|^{2}} .
$$

If the states $\Psi_{[\vec{g}]}^{t}$ are to be good semiclassical states, their overlap (4.13) should be sharply peaked at $g_{1} \approx g_{2}$ in the semiclassical limit $t \rightarrow 0$, as well as they should approximate operators corresponding to gauge-invariant observables well, i.e. they should satisfy the gaugeinvariant version of the Ehrenfest property (3.5). In these two conditions, the semiclassical properties of the states are encoded. They simply amount to the fact that, in the limit $t \rightarrow 0$, a state labeled by a classical, gauge-invariant phase-space point $[\vec{g}]$ approaches the classical state given by this point. In particular, taking expectation values of a quantum observable in this state amounts to evaluation of the corresponding classical observable. Furthermore, the overlap between states labeled with different phase-space points $\left[\vec{g}_{1}\right],\left[\vec{g}_{2}\right]$ is nearly zero, i.e. quantum fluctuations between different states become small.

As we have already reported in section 3 , the complexifier coherent states have these properties, and thus are viable semiclassical states for the gauge variant, i.e. kinematical Hilbert space, approximating gauge-variant classical observables. Establishing analogous properties for the gauge-invariant coherent states, resembling the gauge-invariant physical systems, is the main purpose of this paper. The rest of this work will be devoted to investigate the gaugeinvariant inner product, in order to show the peakedness properties of the gauge-invariant overlap (4.13). Note that, as soon as peakedness properties are established, the Ehrenfest 
property follows immediately from the fact that the corresponding property (3.5) holds for the CCS: assume further that the corresponding gauge-invariant coherent states $\Psi_{[g]}^{t}=\mathcal{P} \psi_{g}^{t}$ have the peakedness property, i.e. $I^{t}\left(\left[g^{\prime}\right],[g]\right)$ is sharply peaked around $[g]=\left[g^{\prime}\right]$ for small $t$. Then the $\Psi_{[g]}^{t}$ also satisfy the Ehrenfest property for gauge-invariant observables, as we will show in what follows. This argument is in fact independent of the choice of the gauge group $G=S U(2)$, and hence cannot only applied to the case $G=U(1)$, but also to any compact, semisimple Lie group $G$.

Assume that the complexifier coherent states satisfy the Ehrenfest property for some set of functions $F$ on phase space (where $g \in G^{\mathbb{C}}$, and $G^{\mathbb{C}} \simeq T^{*} G$ ), i.e.

$$
\frac{\left\langle\psi_{g^{\prime}}^{t}|\hat{F}| \psi_{g}^{t}\right\rangle}{\left\|\psi_{g^{\prime}}^{t}\right\|\left\|\psi_{g}^{t}\right\|} \approx F(g) \frac{\left\langle\psi_{g^{\prime}}^{t} \mid \psi_{g}^{t}\right\rangle}{\left\|\psi_{g^{\prime}}^{t}\right\|\left\|\psi_{g}^{t}\right\|}(1+O(t)) .
$$

Let now $F$ be such a function that is furthermore gauge invariant. Then $\hat{F}$ commutes with the gauge projector $\mathcal{P}$ (2.6), and one has

$$
\begin{aligned}
\left\langle\Psi_{\left[g^{\prime}\right]}^{t}|\hat{F}| \Psi_{[g]}^{t}\right\rangle & =\left\langle\psi_{g^{\prime}}^{t}|\mathcal{P} \hat{F} \mathcal{P}| \psi_{g}^{t}\right\rangle=\left\langle\psi_{g^{\prime}}^{t}\left|\hat{F} \mathcal{P}^{2}\right| \psi_{g}^{t}\right\rangle \\
& =\left\langle\psi_{g^{\prime}}^{t}|\hat{F} \mathcal{P}| \psi_{g}^{t}\right\rangle=\left\langle\psi_{g^{\prime}}^{t}|\hat{F}| \Psi_{[g]}^{t}\right\rangle .
\end{aligned}
$$

Here we have used $\mathcal{P}^{2}=\mathcal{P}$. Furthermore, since the gauge transformations $k \in G^{V}$ act unitarily, we have $\left\|\psi_{g}^{t}\right\|=\left\|\psi_{\alpha_{k} g}^{t}\right\|$. So, we get

$$
\begin{aligned}
\left\langle\psi_{g^{\prime}}^{t}|\hat{F}| \Psi_{[g]}^{t}\right\rangle & =\int_{G^{V}} \mathrm{~d} \mu_{H}^{\otimes V}(k)\left\langle\psi_{g^{\prime}}^{t}|\hat{F}| \psi_{\alpha_{k} g}^{t}\right\rangle \\
& =\left\|\psi_{g}^{t}\right\|\left\|\psi_{g^{\prime}}^{t}\right\| \int_{G^{V}} \mathrm{~d} \mu_{H}^{\otimes V}(k) \frac{\left\langle\psi_{g^{\prime}}^{t}|\hat{F}| \psi_{\alpha_{k} g}^{t}\right\rangle}{\left\|\psi_{g^{\prime}}^{t}\right\|\left\|\psi_{\alpha_{k} g}^{t}\right\|}
\end{aligned}
$$

But since the complexifier coherent states $\psi_{g}^{t}$ satisfy the Ehrenfest property, the integrand in (4.16) is, for small $t$, close to zero almost everywhere, apart from the points where $\alpha_{k} g \approx g^{\prime}$. So the integrand is basically evaluated at that $k$, and we get

$$
\begin{aligned}
\int_{G^{V}} \mathrm{~d} \mu_{H}^{\otimes V}(k) \frac{\left\langle\psi_{g^{\prime}}^{t}|\hat{F}| \psi_{\alpha_{k} g}^{t}\right\rangle}{\left\|\psi_{g^{\prime}}^{t}\right\|\left\|\psi_{\alpha_{k} g}^{t}\right\|} & \approx F(g) \int_{G^{V}} \mathrm{~d} \mu_{H}^{\otimes V}(k) \frac{\left\langle\psi_{g^{\prime}}^{t} \mid \psi_{\alpha_{k} g}^{t}\right\rangle}{\left\|\psi_{g^{\prime}}^{t}\right\|\left\|\psi_{\alpha_{k} g}^{t}\right\|} \\
& =F(g) \frac{\left\langle\Psi_{\left[g^{\prime}\right]}^{t} \mid \Psi_{[g]}^{t}\right\rangle}{\left\|\psi_{g^{\prime}}^{t}\right\|\left\|\psi_{g}^{t}\right\|},
\end{aligned}
$$

where $\approx$ means that equality holds for $t \rightarrow 0$. With (4.16) and (4.17) we thus get

$$
\frac{\left\langle\Psi_{\left[g^{\prime}\right]}^{t}|\hat{F}| \Psi_{[g]}^{t}\right\rangle}{\left\|\Psi_{\left[g^{\prime}\right]}^{t}\right\|\left\|\Psi_{\left[g^{\prime}\right]}^{t}\right\|} \approx F([g]) \frac{\left\langle\Psi_{\left[g^{\prime}\right]}^{t} \mid \Psi_{[g]}^{t}\right\rangle}{\left\|\Psi_{\left[g^{\prime}\right]}^{t}\right\|\left\|\Psi_{\left[g^{\prime}\right]}^{t}\right\|} .
$$

We have replaced $F(g)$ by $F([g])$, since we demanded $F$ to be gauge invariant, i.e. to depend only on the gauge orbits of $g$, not on $g$ itself. Note that in these considerations, we have pulled the error terms $O(t)$ out of the integral, which is allowed, since the error term is of order $O(t)$ on all of the integration range $S U(2)^{V}$, and $S U(2)^{V}$ is compact. Hence we can replace the errors by their maximal absolute value, pull that out of the integral and still have made only an $O(t)$ error.

These considerations show that as soon as we have established the peakedness properties for the gauge-invariant coherent states, the corresponding Ehrenfest properties automatically follow. We will try to establish these peakedness properties in the rest of this paper. 


\subsection{On the overlap of gauge-invariant coherent states}

The gauge-invariant coherent states can be put into an explicit form [3]. Starting from (4.4), one can perform the gauge integrals (2.6) and arrive at

$$
\Psi_{[\vec{g}]}^{t}(\vec{h})=\sum_{\vec{j}, \vec{I}} \mathrm{e}^{-j_{e_{1}}\left(j_{e_{1}}+1\right) \frac{t}{2}-\cdots-j_{e_{E}}\left(j_{e_{E}}+1\right) \frac{t}{2}} T_{\gamma, \vec{j}, \vec{I}}(\vec{g}) \overline{T_{\gamma, \vec{j}, \vec{I}}(\vec{h})} .
$$

Here, the labels denote a distribution $\vec{j}$ of irreducible representations of $S U$ (2) among the edges of the graph $\gamma$, and $\vec{I}$ denotes a distribution of intertwiners among the vertices of the graph. These $T_{\gamma, \vec{j}, \vec{I}}(\vec{h})$ form an orthonormal basis for the gauge-invariant Hilbert space $\mathcal{P} \mathcal{H}_{\gamma}$. The intertwiners $\vec{I}$, in particular the basis functions $T_{\gamma, \vec{\pi}, \vec{I}}$ can be found by employing the coupling scheme for angular momenta, in particular they contain the $3 N j$-symbols [4, 9]. Although these symbols are known in principle, they become arbitrarily complicated for large graphs. This makes expressions (4.19) rather difficult to handle, in particular it is not clear how to extract information about peakedness properties, apart from the simplest example. This is the reason why we will pursue another way, already suggested in [3].

Since $\mathcal{P}^{2}=\mathcal{P}$, we have

$$
\left\langle\Psi_{\left[\vec{g}_{1}\right]}^{t} \mid \Psi_{\left[\vec{g}_{2}\right]}^{t}\right\rangle=\int_{G^{V}} \mathrm{~d} \mu_{H}^{\otimes V}(\vec{k})\left\langle\psi_{\vec{g}_{1}}^{t} \mid \psi_{\alpha_{\vec{k}} \vec{g}_{2}}^{t}\right\rangle
$$

So the inner product between gauge-invariant coherent states can be obtained by an integral over the inner product between complexifier coherent states. This inner product is given by (3.11) and (3.12).

From the gauge-invariant inner product, we can immediately obtain the overlap between gauge-invariant coherent states by

$$
\frac{\left|\left\langle\Psi_{\left[\vec{g}_{1}\right]}^{t} \mid \Psi_{\left[\vec{g}_{2}\right]}^{t}\right\rangle\right|^{2}}{\left\|\Psi_{\left[\vec{g}_{2}\right]}^{t}\right\|^{2}\left\|\Psi_{\left[\vec{g}_{2}\right]}^{t}\right\|^{2}}=\frac{\left.\left.\left[\int_{G^{V}} \mathrm{~d} \mu_{H}^{\otimes V}(\vec{k})\left\langle\psi_{\vec{g}_{1}}^{t}\right| \psi_{\alpha_{\vec{k}} \vec{g}_{2}}^{t}\right)\right]\left[\int_{G^{V}} \mathrm{~d} \mu_{H}^{\otimes V}(\vec{k})\left\langle\psi_{\vec{g}_{2}}^{t}\right| \psi_{\alpha_{\vec{k}} \vec{g}_{1}}^{t}\right)\right]}{\left.\left[\int_{G^{V}} \mathrm{~d} \mu_{H}^{\otimes V}(\vec{k})\left\langle\psi_{\vec{g}_{1}}^{t} \mid \psi_{\alpha_{\vec{k}} \vec{g}_{1}}^{t}\right\rangle\right]\left[\int_{G^{V}} \mathrm{~d} \mu_{H}^{\otimes V}(\vec{k})\left\langle\psi_{\vec{g}_{2}}^{t}\right| \psi_{\alpha_{\vec{k}} \vec{g}_{2}}^{t}\right)\right]}
$$

This form of the inner product between gauge-invariant coherent states is not explicit, since it still contains the gauge integral. But this form has some advantages over the corresponding formula using the explicit form of the gauge-invariant coherent states (4.19). First, we will see that we can, by some gauge-fixing procedure, extract the gauge-invariant quantities that label the gauge orbits. These not only have a geometrical interpretation, but the procedure also allows for a numerical investigation that enables us to show that the gauge-invariant coherent states are peaked on these gauge-invariant quantities. Second, starting from (4.21), we will be able to prove a general theorem about the peakedness of these states on the singular points of the space of gauge-invariant quantities.

So, four integrals of the type of (4.20) have to computed. It is exactly these integrals that turn out to be not solvable in a closed form for most graphs, apart from the simplest ones. In particular, we will be able to compute the overlap analytically for the 1-flower graph, but on the 2-flower, the 3-bridge and the tetrahedron graph, we will employ numerical integration of (4.21), in order to investigate the overlap. After that, we will demonstrate some qualitative properties of the overlap (4.21) in section 5 .

\subsection{The 1-flower graph}

In the following, we will consider the gauge-invariant coherent states on simple graphs for the case of $G=S U$ (2). In particular, we will have to evaluate integrals of the kind (4.20). This will not be possible analytically for most cases, but we will do this where we can, and use numerics in all other cases. 


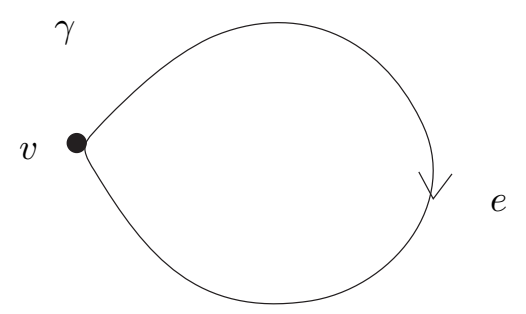

Figure 1. A 1-flower graph $\gamma$, which consists of only one vertex $v$ and only one edge $e$, which starts and ends at $v$.

We start with the 1-flower graph (see figure 1). Here we will be able to perform the results analytically, since the intertwiners for this graph are just the traces.

With (4.19), we get

$$
\Psi_{[g]}^{t}(h)=\sum_{j \in \frac{1}{2} \mathbb{N}} \mathrm{e}^{-j(j+1) \frac{t}{2}} \operatorname{tr}_{j}(g) \operatorname{tr}_{j}(h) .
$$

The inner product between two such coherent states labeled by $\left[g_{1}\right]$ and $\left[g_{2}\right]$ is then given by

$$
\left\langle\Psi_{\left[g_{1}\right]}^{t} \mid \Psi_{\left[g_{2}\right]}^{t}\right\rangle=\sum_{j \in \frac{1}{2} \mathbb{N}} \mathrm{e}^{-j(j+1) t} \overline{\operatorname{tr}_{j}\left(g_{1}\right)} \operatorname{tr}_{j}\left(g_{2}\right) .
$$

Consider the $j=\frac{1}{2}$ representation of $S L(2, \mathbb{C})$. There are invertible $2 \times 2$ matrices $\Phi_{1}, \Phi_{2}$, such that

$\Phi_{2} \pi_{\frac{1}{2}}\left(g_{2}\right) \Phi_{2}^{-1}=\left(\begin{array}{ll}\lambda & 0 \\ 0 & \lambda^{-1}\end{array}\right), \quad \Phi_{1} \pi_{\frac{1}{2}}\left(g_{1}\right) \Phi_{1}^{-1}=\left(\begin{array}{ll}\mu & 0 \\ 0 & \mu^{-1}\end{array}\right)$

for nonzero $\lambda, \mu \in \mathbb{C}$. By an explicit formulation of the irreducible representations of $S L(2, \mathbb{C})$, one gets [2]

$$
\operatorname{tr} \pi_{j}\left(g_{2}\right)=\frac{\lambda^{2 j+1}-\lambda^{-2 j-1}}{\lambda-\lambda^{-1}}, \quad \operatorname{tr} \pi_{j}\left(g_{1}\right)=\frac{\mu^{2 j+1}-\mu^{-2 j-1}}{\mu-\mu^{-1}} .
$$

Note that these expressions are invariant under the change of $\lambda \rightarrow \lambda^{-1}$ or $\mu \rightarrow \mu^{-1}$, as are the choices of $\Phi_{1}, \Phi_{2}$ in (4.24).

Write $\lambda=\mathrm{e}^{\mathrm{i} z}$ and $\mu=\mathrm{e}^{\mathrm{i} w}$, then we get

$$
\begin{aligned}
\left\langle\Psi_{\left[g_{1}\right]}^{t} \mid \Psi_{\left[g_{2}\right]}^{t}\right\rangle & =\sum_{j \in \frac{1}{2} \mathbb{N}} \mathrm{e}^{-j(j+1) t} \frac{\mathrm{e}^{-\mathrm{i}(2 j+1) \bar{w}}-\mathrm{e}^{\mathrm{i}(2 j+1) \bar{w}}}{\mathrm{e}^{-\mathrm{i} \bar{w}}-\mathrm{e}^{\mathrm{i} \bar{w}}} \frac{\mathrm{e}^{\mathrm{i}(2 j+1) z}-\mathrm{e}^{-\mathrm{i}(2 j+1) z}}{\mathrm{e}^{\mathrm{i} z}-\mathrm{e}^{-\mathrm{i} z}} \\
& =\mathrm{e}^{\frac{t}{4}} \sum_{n=1}^{\infty} \mathrm{e}^{-n^{2} \frac{t}{4}} \frac{\mathrm{e}^{-\mathrm{i} n \bar{w}}-\mathrm{e}^{\mathrm{i} n \bar{w}}}{\mathrm{e}^{-\mathrm{i} \bar{w}}-\mathrm{e}^{\mathrm{i} \bar{w}} \frac{\mathrm{e}^{-\mathrm{i} n z}}{\mathrm{e}^{\mathrm{i} z}-\mathrm{e}^{-\mathrm{i} z}}} \\
& =\frac{1}{2} \mathrm{e}^{\frac{t}{4}} \sum_{n \in \mathbb{Z}} \mathrm{e}^{-n^{2} \frac{t}{4}} \frac{\mathrm{e}^{-\mathrm{i} n \bar{w}}-\mathrm{e}^{\mathrm{i} n \bar{w}}}{\mathrm{e}^{-\mathrm{i} \bar{w}}-\mathrm{e}^{\mathrm{i} \bar{w}}} \frac{\mathrm{e}^{\mathrm{i} n z}-\mathrm{e}^{-\mathrm{i} n z}}{\mathrm{e}^{\mathrm{i} z}-\mathrm{e}^{-\mathrm{i} z}} .
\end{aligned}
$$

By the Poisson summation formula,

$$
\sum_{n \in \mathbb{Z}} \mathrm{e}^{-n^{2} \frac{t}{4}} \mathrm{e}^{\mathrm{i} n A}=2 \sqrt{\frac{\pi}{t}} \sum_{n \in \mathbb{Z}} \mathrm{e}^{-\frac{(A+2 \pi n)^{2}}{t}} .
$$


With (4.26), (4.25) can be rewritten as follows:

$$
\left\langle\Psi_{\left[g_{1}\right]}^{t} \mid \Psi_{\left[g_{2}\right]}^{t}\right\rangle=\mathrm{e}^{\frac{t}{4}} \sqrt{\frac{\pi}{t}} \sum_{n \in \mathbb{Z}} \frac{\mathrm{e}^{-\frac{(\bar{w}-z-2 \pi n)^{2}}{t}}-\mathrm{e}^{-\frac{(\bar{w}+z-2 \pi n)^{2}}{t}}}{\left(\mathrm{e}^{-\mathrm{i} \bar{w}}-\mathrm{e}^{\mathrm{i} \bar{w}}\right)\left(\mathrm{e}^{\mathrm{i} z}-\mathrm{e}^{-\mathrm{i} z}\right)} .
$$

If we choose $z$ and $w$ to lie inside the strip $[-\pi, \pi] \times \mathrm{i} \mathbb{R}$, the inner product can be approximated by

$$
\left\langle\Psi_{\left[g_{1}\right]}^{t} \mid \Psi_{\left[g_{2}\right]}^{t}\right\rangle=\mathrm{e}^{\frac{t}{4}} \sqrt{\frac{\pi}{t}} \frac{\mathrm{e}^{-\frac{(\bar{w}-z)^{2}}{t}}-\mathrm{e}^{-\frac{(\bar{w}+z)^{2}}{t}}}{\left(\mathrm{e}^{-\mathrm{i} \bar{w}}-\mathrm{e}^{\mathrm{i} \bar{w}}\right)\left(\mathrm{e}^{\mathrm{i} z}-\mathrm{e}^{-\mathrm{i} z}\right)}\left(1+O\left(t^{\infty}\right)\right) .
$$

With

$$
\mathrm{e}^{A}-\mathrm{e}^{B}=2 \mathrm{e}^{\frac{A+B}{2}} \sinh \frac{A-B}{2}
$$

this can be put into the form

$$
\left\langle\Psi_{\left[g_{1}\right]}^{t} \mid \Psi_{\left[g_{2}\right]}^{t}\right\rangle=\frac{1}{2} \mathrm{e}^{\frac{t}{4}} \sqrt{\frac{\pi}{t}} \frac{\mathrm{e}^{-\frac{\bar{w}^{2}+z^{2}}{t}} \sinh \frac{2 \bar{w} z}{t}}{\sin \bar{w} \sin z}\left(1+O\left(t^{\infty}\right)\right),
$$

which, for the overlap, gives

$I^{t}\left(\left[g_{1}\right],\left[g_{2}\right]\right)=\frac{\left|\left\langle\Psi_{\left[g_{1}\right]}^{t} \mid \Psi_{\left[g_{2}\right]}^{t}\right\rangle\right|^{2}}{\left\|\Psi_{\left[g_{1}\right]}^{t}\right\|^{2}\left\|\Psi_{\left[g_{2}\right]}^{t}\right\|^{2}}=\frac{\sinh \frac{2 \bar{w} z}{t} \sinh \frac{2 w \bar{z}}{t}}{\sinh \frac{2|w|^{2}}{t} \sinh \frac{2|z|^{2}}{t}}\left(1+O\left(t^{\infty}\right)\right)$.

Note that the complex numbers $z, w$ appearing here are related to the labels of the coherent states via $2 \cos z=\operatorname{tr} g_{2}$ and $2 \cos w=\operatorname{tr} g_{1}$. Note also how the gauge-invariant coherent states only depend on the trace $\operatorname{tr}_{j}(g)$. Since the only invariant information under conjugation in $S L(2, \mathbb{C}): g \rightarrow k g k^{-1}$ is the trace, we see that the gauge-invariant coherent states on the 1 -flower graph only depend on the conjugation classes, i.e. elements in $S L(2, \mathbb{C}) / S L(2, \mathbb{C})$, as has been stated in the last section.

We plot the overlap between $\Psi_{\cos w}^{t}$ and $\Psi_{\cos z}^{t}$, for $w=1+\mathrm{i}$, depending on $z$, with $t=1$.

As one can see in figure 2, the overlap is peaked at $z= \pm w$, as should be the case, since $z$ and $w$ are, if both in the region $(-\pi, \pi] \times \mathrm{i} \mathbb{R}$, only determined up to a sign. The overlap profile is a Gaussian, as can readily be seen.

In the second plot, shown in figure 3 , we note the significant broader peak around $z=0$. This is not a Gaussian anymore. Rather, by performing limits carefully in expression (4.29), one can see that it is actually a $2|z|^{2} /\left(t \sinh \left(2|z|^{2} / t\right)\right)$ profile, which is much broader than a Gaussian. For $|z| \rightarrow 0$, this goes as $1-\frac{2}{3 t^{2}}|z|^{4}$, rather than as $1-k|z|^{2}$, as would have been expected from a Gaussian.

This significant change in the peak profile is simply due to the fact that the space of equivalence classes is no manifold any longer, but contains singularities: Namely those at $g= \pm \mathbb{1} \in S L(2, \mathbb{C})$, that are the only points where the gauge group does not act effectively on the orbits. It is exactly this feature that we will also encounter in the other examples. We will also be able to provide a general result concerning this property of gauge-invariant coherent states labeled by degenerate gauge orbits.

\subsection{The 2-flower graph}

We now turn to the more complicated case of a 2-flower graph, which consists of one vertex $v$ and two edges, both starting and ending at $v$. Although this graph is still much simpler than any graph of relevance for LQG, the gauge-invariant coherent states are sufficiently complicated, 


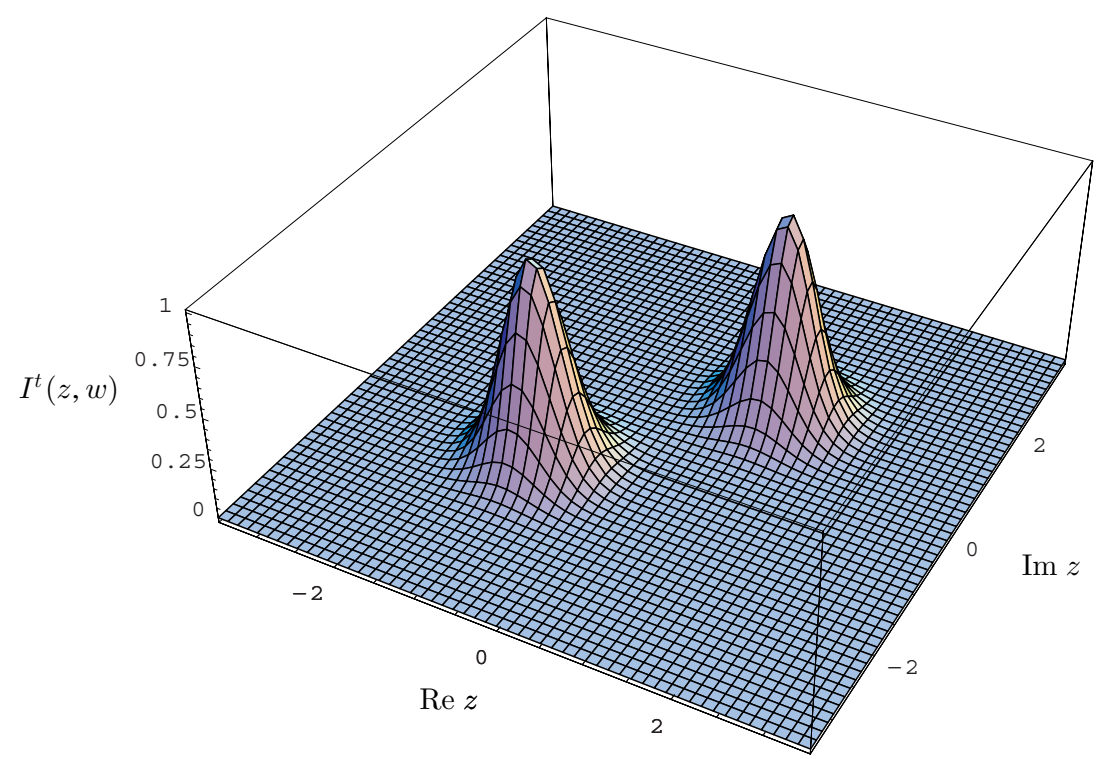

Figure 2. Overlap $I^{t}(z, w)$ between the gauge-invariant coherent states $\Psi_{\cos z}^{t}$ and $\Psi_{\cos w}^{t}$ with $w=1+\mathrm{i}, t=1$, depending on $z$.

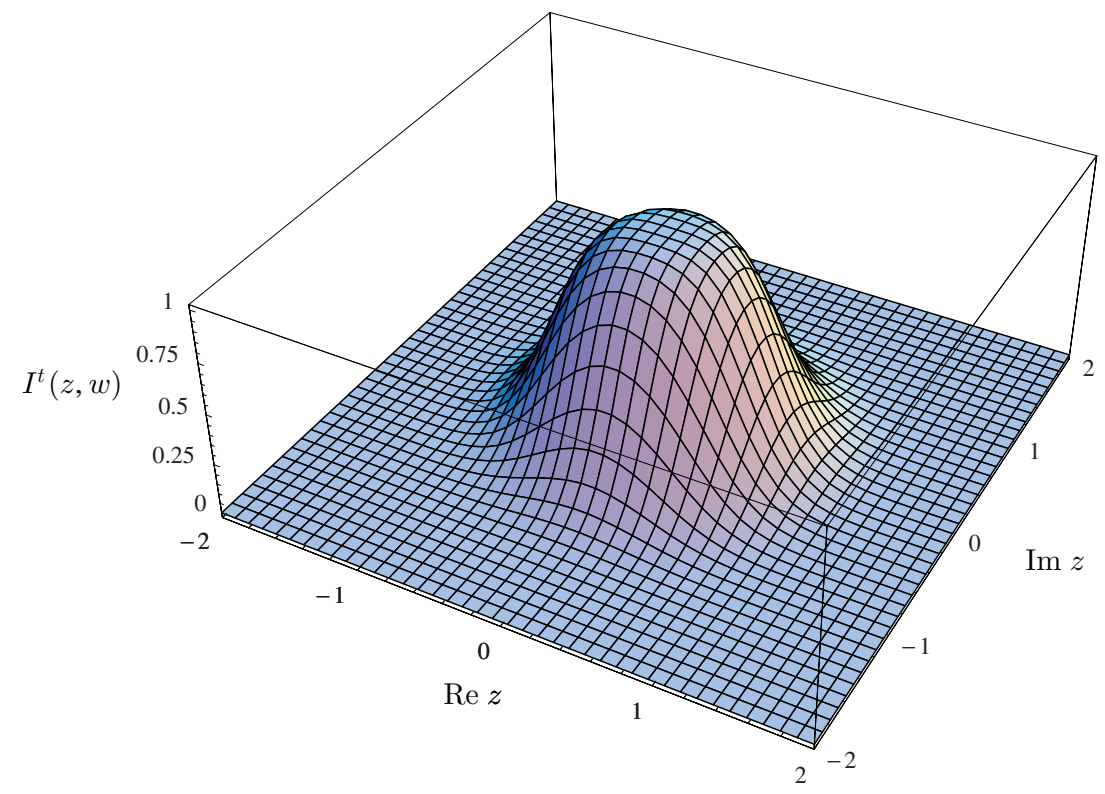

Figure 3. Overlap between the gauge-invariant coherent states $\Psi_{\cos z}^{t}$ and $\Psi_{\cos w}^{t}$ with $w=0, t=1$, depending on $z$.

such that the overlap cannot be calculated analytically any more. Starting with the form (4.19) for the gauge-invariant coherent states, the intertwiner for the 2-flower graph can be computed, as can the basis $T_{\gamma \vec{j}, \vec{I}}$ for the gauge-invariant coherent states. In particular, the gauge-invariant 
coherent states are given by

$$
\begin{aligned}
\Psi_{\left[g_{1}, g_{2}\right]}^{t}\left(h_{1}, h_{2}\right) & =\sum_{j_{1}, j_{2} \in \frac{1}{2} \mathbb{Z}} \mathrm{e}^{-j_{1}\left(j_{1}+1\right) \frac{t}{2}-j_{2}\left(j_{2}+1\right) \frac{t}{2}}\left(2 j_{1}+1\right)\left(2 j_{2}+1\right) \\
& \times \sum_{J=\left|j_{1}-j_{2}\right|}^{j_{1}+j_{2}} \sum_{M, N=-J}^{J} C_{j_{1} j_{2} m_{1} m_{2}}^{J M} C_{j_{1} j_{2} n_{1} n_{2}}^{J M} \pi_{j_{1}}\left(h_{1}\right)_{m_{1} n_{1}} \pi_{j_{2}}\left(h_{2}\right)_{m_{2} n_{2}} \\
& \times C_{j_{1} j_{2} \tilde{m}_{1} \tilde{m}_{2}}^{J N} C_{j_{1} j_{2} \tilde{n}_{1} \tilde{n}_{2}}^{J N} \pi_{j_{1}}\left(g_{1}^{c}\right)_{\tilde{m}_{1} \tilde{n}_{1}} \pi_{j_{2}}\left(g_{2}^{c}\right)_{\tilde{m}_{2} \tilde{n}_{2}} .
\end{aligned}
$$

Unfortunately, the occurring Clebsch-Gordan coefficients make the use of the Poisson summation formula too complicated, such that one cannot hope to rewrite (4.30) as a sum over $n$, such that only the $n=0$ term dominates in the $t \rightarrow 0$ limit, which simplified the analysis of the overlap tremendously in the case of $S U(2)$-complexifier- and $U(1)$-gauge-invariant coherent states.

So, we will perform a different route. We will start with formula (4.21) for the overlap of the gauge-invariant coherent states. Thus, we need to perform the gauge integrals

$$
\begin{aligned}
\left\langle\Psi_{\left[g_{1}, g_{2}\right]}^{t} \mid \Psi_{\left[h_{1}, h_{2}\right]}^{t}\right\rangle & \sim \int_{S U(2)} \mathrm{d} \mu_{H}(k) \sum_{n_{1}, n_{2} \in \mathbb{Z}} \frac{f_{1}(k)-2 \pi \mathrm{i} n_{1}}{\sinh \left(f_{1}(k)-2 \pi \mathrm{i} n_{1}\right)} \frac{f_{2}(k)-2 \pi \mathrm{i} n_{2}}{\sinh \left(f_{2}(k)-2 \pi \mathrm{i} n_{2}\right)} \\
& \times \exp \left(\frac{\left.\left(f_{1}(k)-2 \pi \mathrm{i} n_{1}\right)^{2}+\left(f_{2}(k)-2 \pi \mathrm{i} n_{2}\right)^{2}\right)}{t}\right)
\end{aligned}
$$

with

$$
\cosh f_{1}(k)=\frac{1}{2} \operatorname{tr}\left(g_{1}^{\dagger} k h_{1} k^{-1}\right) \quad \cosh f_{2}(k)=\frac{1}{2} \operatorname{tr}\left(g_{2}^{\dagger} k h_{2} k^{-1}\right) .
$$

Unfortunately, this gauge integral is, although only an integral over $S U(2)$, still too complicated to compute analytically. Even the attempt to obtain an asymptotic expression for the limit $t \rightarrow 0$, i.e. by employing the method of stationary phase, fails, because of the complicated structure of (4.32). In trying to find the points where the exponent $\left(f_{1}(k)-2 \pi \mathrm{i} n_{1}\right)^{2}+\left(f_{2}(k)-2 \pi \mathrm{i} n_{2}\right)^{2}$ becomes stationary, one can proceed quite far, in fact one can locate these points to be in certain one-dimensional submanifolds of $S U(2)$, depending on the $g_{1,2}, h_{1,2}$. But calculating the exact position of the stationary points eventually lead to transcendent equations, which could not be solved.

What remains to do for us is to compute the integrand (4.31) numerically, and thus perform a numerical analysis for the overlap (4.21). In order to show that the gauge-invariant coherent states are really peaked on gauge-invariant quantities, first we have to identify these quantities, i.e. gauge-fix the integrand in (4.31).

Lemma (4.1) allows us to shift the integrand in (4.31) not only by elements of $S U$ (2) from the right and from the left, which is clear from the bi-invariance of the Haar measure, but also allows us to shift the integrand by elements of $S L(2, \mathbb{C})$. Note the analogy to shifting the integral of an analytic function over $\mathbb{R}$ into the complex plane.

Now let us investigate expressions (4.32). By writing

$$
g_{j}=\exp \left(\mathrm{i} \vec{\sigma} \cdot \vec{w}_{j}\right), \quad h_{j}=\exp \left(\mathrm{i} \vec{\sigma} \cdot \vec{z}_{j}\right), \quad j=1,2
$$

with $\vec{z}_{i}, \vec{w}_{i} \in \mathbb{C}^{3}$, we see that shifting the integration variable by $k \rightarrow \tilde{g}^{\dagger} k \tilde{h}$ changes (4.32) to

$$
\frac{1}{2} \operatorname{tr}\left(g_{j}^{\dagger} k h_{j} k^{-1}\right) \longrightarrow \frac{1}{2} \operatorname{tr}\left(\left(g_{j}^{\prime}\right)^{\dagger} k\left(h_{j}^{\prime}\right) k^{-1}\right)
$$

with

$$
\begin{aligned}
g_{j}^{\prime} & =\tilde{g} g_{j} \tilde{g}^{-1}=\exp \left(\mathrm{i} \vec{\sigma} \cdot G \vec{w}_{j}\right) \\
h_{j}^{\prime} & =\tilde{h} h_{j} \tilde{h}^{-1}=\exp \left(\mathrm{i} \vec{\sigma} \cdot H \vec{z}_{j}\right), \quad j=1,2
\end{aligned}
$$


with $G, H \in O(3, \mathbb{C})$. Note that here we encounter the vector representation of $S L(2, \mathbb{C})$ as orthogonal rotations in $\mathbb{C}^{3}$. In particular, we have

$$
G=\pi_{1}(\tilde{g}), \quad H=\pi_{1}(\tilde{h}) .
$$

Here we get a first glimpse at the gauge-invariant information contained in a pair $\left(g_{1}, g_{2}\right)$. A gauge-invariant state is not labeled by this pair, but rather by this pair modulo conjugation with elements in $\tilde{g} \in S L(2, \mathbb{C})$. If $\left(g_{1}, g_{2}\right)$ are well away from the negative Hermitean elements in $S L(2, \mathbb{C})$, we can talk about vectors in $\mathbb{C}^{3}$ rather than $S L(2, \mathbb{C})$ elements, which illustrate the facts better: Instead of $g_{j}=\exp \left(\mathrm{i} \vec{\sigma} \cdot \vec{z}_{j}\right) \in S L(2, \mathbb{C})$, consider $\vec{z}_{j} \in \mathbb{C}^{3}$ themselves. Rather than by two vectors $\vec{z}_{1}, \vec{z}_{2} \in \mathbb{C}^{3}$, the gauge-invariant states are labeled by these vectors modulo rotation in $\mathbb{C}^{3}$. Here we mean rotations that leave $\vec{z}_{j} \cdot \vec{z}_{j}$, rather than $\vec{z}_{j} \cdot \vec{z}_{j}$ invariant, i.e. we talk about orthogonal rotations, not unitary rotations.

Geometrically, this can be seen as the description of a parallelogram in $\mathbb{C}^{3}$, where one point is fixed at $\overrightarrow{0} \in \mathbb{C}^{3}$, modulo rotations around that point. Such a parallelogram is just given by three (complex) numbers: the complex length of two of its sides and the complex angle between them. In the following, we will gauge-fix the integrand (4.31) such that these gauge-invariant quantities will explicitly be visible. We will then present a number of plots which confirm that the overlap between gauge-invariant coherent states is indeed peaked on these three numbers, i.e. the overlap between two gauge-invariant coherent states, where only one of these three parameters differs, is close to zero.

To simplify our analysis, we only consider elements $g_{j}, h_{j}$, such that the complex length of neither of the associated vectors is zero:

$$
\vec{z}_{j} \cdot \vec{z}_{j} \neq 0 \neq \vec{w}_{j} \cdot \vec{w}_{j}, \quad j=1,2 .
$$

Note that $O(3, \mathbb{C})$ does not only map the set of vectors $\{\vec{z} \cdot \vec{z}=a\}$ into itself for each $a \in \mathbb{C}$, but also acts simply transitively on them, except for the case of $a=0$, where the zero vector has to be excluded. Thus, we use the freedom of $G, H \in O(3, \mathbb{C})$ to rotate $\vec{w}_{1}$ and $\vec{z}_{2}$ into the 3-direction. In particular, there are $G, H \in O(3, \mathbb{C})$ such that

$$
G \vec{w}_{1}=\left(\begin{array}{c}
0 \\
0 \\
w_{1}
\end{array}\right), \quad H \vec{z}_{2}=\left(\begin{array}{c}
0 \\
0 \\
z_{2}
\end{array}\right)
$$

with

$$
w_{1}^{2}=\vec{w}_{1} \cdot \vec{w}_{1}, \quad z_{2}^{2}=\vec{z}_{2} \cdot \vec{z}_{2} .
$$

Of course, $\vec{z}_{1}, \vec{w}_{2}$ have also been changed by this transformation to $\vec{z}_{1}^{\prime}, \vec{w}_{2}^{\prime}$. The remaining freedom to rotate these vectors without destroying the gauge-fixed (4.33) is effectively an $O(2, \mathbb{C})$ rotation in the complex 1-2-plane. We can use this remaining freedom to rotate both $\vec{z}_{1}^{\prime}, \vec{w}_{2}^{\prime}$ out of the 1-direction. In particular, there are rotations $G^{\prime}, H^{\prime} \in O(3, \mathbb{C})$ such that

$$
\begin{gathered}
G^{\prime}\left(\begin{array}{c}
0 \\
0 \\
w_{1}
\end{array}\right)=\left(\begin{array}{c}
0 \\
0 \\
w_{1}
\end{array}\right)=: \vec{w}_{1}, \quad H^{\prime}\left(\begin{array}{c}
0 \\
0 \\
z_{2}
\end{array}\right)=\left(\begin{array}{c}
0 \\
0 \\
z_{2}
\end{array}\right)=: \vec{z}_{2} \\
G^{\prime} \vec{w}_{2}^{\prime}=\left(\begin{array}{c}
0 \\
w_{2} \sin \chi \\
w_{2} \cos \chi
\end{array}\right)=: \vec{w}_{2}, \quad H^{\prime} \vec{z}_{1}^{\prime}=\left(\begin{array}{c}
0 \\
z_{1} \sin \theta \\
z_{1} \cos \theta
\end{array}\right)=: \vec{z}_{1}
\end{gathered}
$$

with $\theta, \chi \in \mathbb{C}$. With this, the integrand is completely gauge-fixed, apart from discrete symmetries having to do with the fact that the $z_{j}, w_{j}$ and $\theta, \chi$ are defined only up to a sign. 


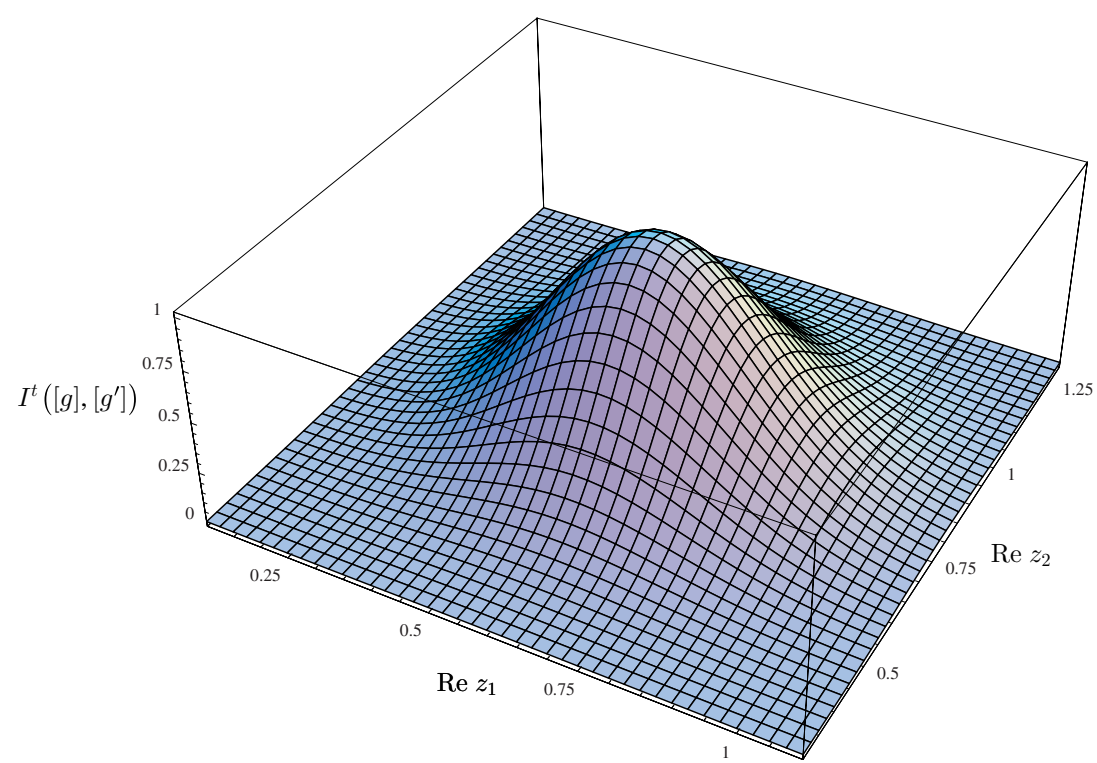

Figure 4. Overlap (4.36) between the two coherent states $\Psi_{[g]}^{t}$ and $\Psi_{\left[g^{\prime}\right]}^{t}$ : one with gauge-invariant data $w_{1}=0.6, w_{2}=0.8, \chi=0$, the other one with $\theta=0$, depending on $z_{1}$ and $z_{2}$.

With this, the integral (4.31) reads

$$
\begin{gathered}
\left\langle\Psi_{\left[g_{1}, g_{2}\right]}^{t} \mid \Psi_{\left[h_{1}, h_{2}\right]}^{t}\right\rangle \sim \int_{S U(2)} \mathrm{d} \mu_{H}(k) \sum_{n_{1}, n_{2} \in \mathbb{Z}} \frac{f_{1}(k)-2 \pi \mathrm{i} n_{1}}{\sinh \left(f_{1}(k)-2 \pi \mathrm{i} n_{1}\right)} \frac{f_{2}(k)-2 \pi \mathrm{i} n_{2}}{\sinh \left(f_{2}(k)-2 \pi \mathrm{i} n_{2}\right)} \\
\times \exp \left(\frac{\left.\left(f_{1}(k)-2 \pi \mathrm{i} n_{1}\right)^{2}+\left(f_{2}(k)-2 \pi \mathrm{i} n_{2}\right)^{2}\right)}{t}\right)
\end{gathered}
$$

with

$$
\begin{aligned}
& \cosh f_{1}(k)=\cos z_{1} \cos \bar{w}_{1}+\sin z_{1} \sin \bar{w}_{1} \cos (\tilde{\theta}(k)) \\
& \cosh f_{2}(k)=\cos z_{2} \cos \bar{w}_{2}+\sin z_{2} \sin \bar{w}_{2} \cos (\overline{\tilde{\chi}}(k)),
\end{aligned}
$$

where $\tilde{\theta}(k)$ is the (complex) angle between $\overline{\vec{w}}_{1}$ and $\pi_{1}(k) \vec{z}_{1}$, and $\tilde{\chi}(k)$ is that between $\overline{\vec{w}}_{2}$ and $\pi_{1}(k) \vec{z}_{2}$.

Now the inner product between the two coherent states is only dependent on the triples $\left(z_{1}, z_{2}, \theta\right)$ and $\left(w_{1}, w_{2}, \chi\right)$, which constitutes the gauge-invariant information of the two gaugeinvariant coherent states. With (4.21) one can compute the overlap from the inner products given by (4.34) with (4.35). We have done this numerically for a couple of examples. In each case, we have fixed the triple $\left(w_{1}, w_{2}, \chi\right)$ and four of the six real parameters in $\left(z_{1}, z_{2}, \theta\right)$, and plotted the overlap

$$
I^{t}\left([g],\left[g^{\prime}\right]\right)=\frac{\left.\left|\left\langle\Psi_{\left(w_{1}, w_{2}, \chi\right)}^{t}\right| \Psi_{\left(z_{1}, z_{2}, \theta\right)}^{t}\right)\right|^{2}}{\left\|\Psi_{\left(w_{1}, w_{2}, \chi\right)}^{t}\right\|^{2}\left\|\Psi_{\left(z_{1}, z_{2}, \theta\right)}^{t}\right\|^{2}} .
$$

The first plot, shown in figure 4, the overlap between a coherent state labeled by $w_{1}=0.6, w_{2}=0.8, \chi=0$ and one labeled by $\theta=0$ and variable $z_{1}, z_{2}$. The width of both states have been chosen to be $t=0.2$. As one can see, the overlap is peaked at the point where both label sets coincide. Also, the shape resembles that of a Gaussian quite well. 


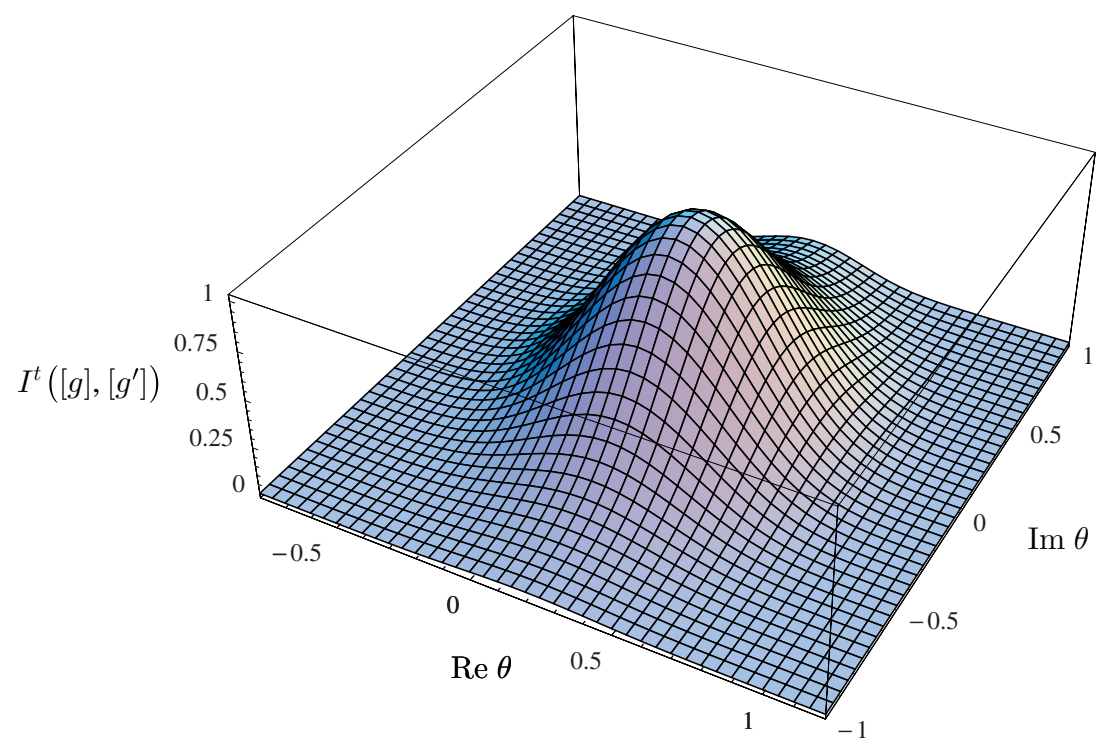

Figure 5. Overlap between the two coherent states $\Psi_{[g]}^{t}$ and $\Psi_{\left[g^{\prime}\right]}^{t}$ : one with gauge-invariant data $w_{1}=\mathrm{i}, w_{2}=1, \chi=0.2$, the other one with $z_{1}=\mathrm{i}$ and $z_{2}=1$, depending on $\theta$.

The second plot, shown in figure 5, shows that the gauge-invariant coherent states are also peaked in the complex angles $\theta, \chi$. The plot shows the overlap between a coherent state labeled with $w_{1}=\mathrm{i}, w_{2}=1, \chi=0.2$, and one labeled by $z_{1}=\mathrm{i}, z_{2}=1$ and $\theta$, where the overlap depending on the complex parameter $\theta$ is shown. The semiclassicality parameter was again chosen to be $t=0.2$.

Again, one can see that in the vicinity of the point where the label sets coincide, the overlap behaves nearly as a Gaussian. Further away, though, the overlap differs slightly from a Gaussian (note the 'bump' at $\theta \approx I$ ).

Apparently, this is due to the fact that $\left(z_{1}, z_{2}, \theta\right)$ are just one set of gauge-invariant labels of a state. There are, of course, infinitely many other equivalent ones, depending on how one gauge-fixes the integrand (4.31). In most of them, the overlap will not exactly be a Gaussian, but will have a rather generic form such as

$$
I^{t}\left(w_{1}, w_{2}, \chi, z_{1}, z_{2}, \theta\right)=\exp \left(-\frac{F\left(w_{1}, w_{2}, \chi, z_{1}, z_{2}, \theta\right)}{t}\right)
$$

with $F=O\left(\left(z_{1}-w_{1}\right)^{2},\left(z_{2}-w_{2}\right)^{2},(\theta-\chi)^{2}\right)$. Of course, as $t \rightarrow 0, F$ can be more and more approximated by its series up to the quadratic order; hence, as $t \rightarrow 0$, the overlap becomes more and more a Gaussian.

The third plot, shown in figure 6, does not show a Gaussian peak. In this plot, we have chosen one state to be peaked at $[\mathbb{1}, \mathbb{1}]$, i.e. $w_{1}=w_{2}=\chi=0$, as well as $\theta=0$ and $t=0.2$.

One can immediately see the 'plateau' around the point where both label sets coincide. Here, although the overlap decreases as the two label sets start to disagree, it decreases qualitatively slower than in the other cases. In particular, the overlap behaves rather like $\exp \left(-x^{4}\right)$, than $\exp \left(-x^{2}\right)$. This behavior does not change as $t \rightarrow 0$. Rather, the function $F$ in (4.36) goes like $O\left(\left|z_{1}\right|^{4},\left|z_{2}\right|^{4},|\theta|^{4}\right)$. 


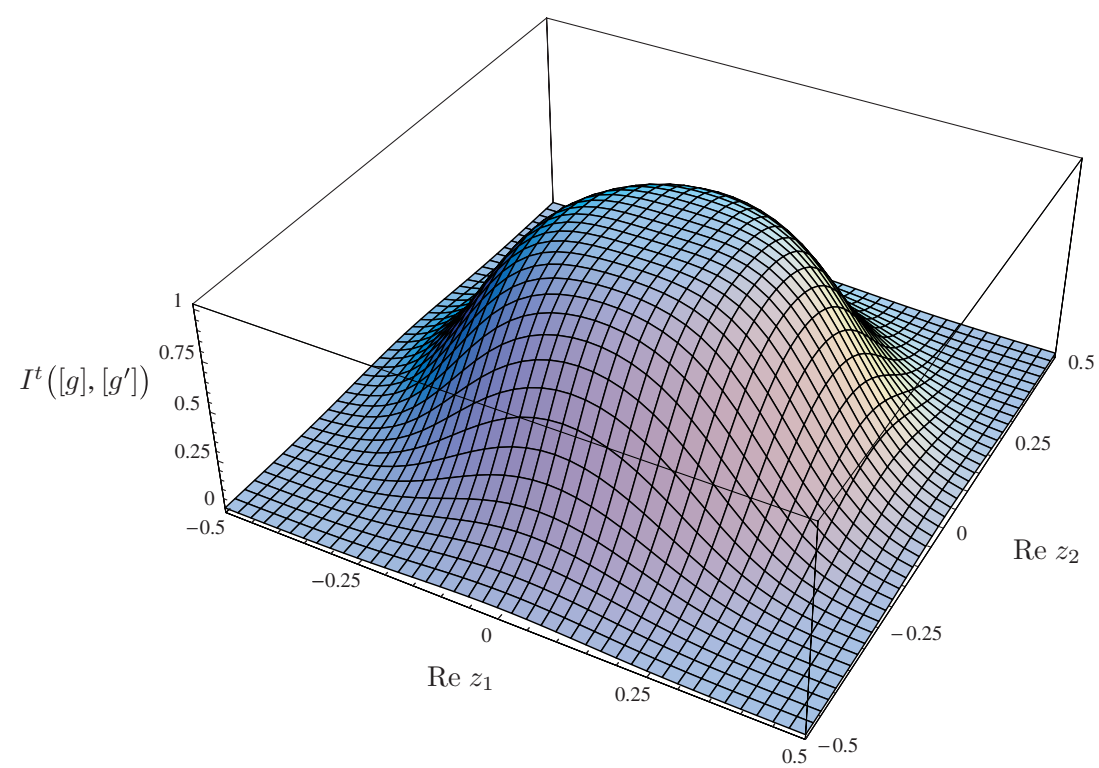

Figure 6. Overlap between the two coherent states $\Psi_{[g]}^{t}$ and $\Psi_{\left[g^{\prime}\right]}^{t}$ : one with gauge-invariant data $w_{1}=0, w_{2}=0, \chi=0$ (corresponding to $[g]=[\mathbb{1}, \mathbb{1}]$ ), the other one with $\theta=0$, depending on $z_{1}$ and $z_{2}$.

Note that this phenomenon has already been encountered in the case of the 1-flower graph. The overlap (4.29) is, in the limit of $w=0$, equal to

$$
\frac{\mid\left\langle\Psi_{[1]}^{t}\left|\Psi_{\cos z}\right|^{2}\right.}{\left\|\Psi_{[1]}^{t}\right\|^{2}\left\|\Psi_{\cos z}^{t}\right\|^{2}}=\frac{2|z|^{2} / t}{\sinh \left(2|z|^{2} / t\right)}\left(1+O\left(t^{\infty}\right)\right)
$$

which goes as $\sim 1-\frac{2}{3 t^{2}}|z|^{4}$ as $z \rightarrow 0$.

So, although peaked at the point indicated by the label set, the state $\Psi_{[1, \mathbb{1}}^{t}$, with $(\mathbb{1}, \mathbb{1}) \in S L(2, \mathbb{C})^{2}$, is much less peaked than generic gauge-invariant states. This will be proven explicitly in section 5.1.

\subsection{The 3-bridge graph}

After the 2-flower graph, we will proceed with the 3-bridge (or sunset) graph, which consists of two vertices $v_{1}$ and $v_{2}$ and three edges $e_{1}, e_{2}, e_{3}$, all beginning at $v_{1}$ and ending at $v_{2}$.

As for the 2-flower graph, the gauge-invariant coherent states on the 3-bridge graph are too complicated to compute the overlap such that statements about peakedness properties can be made. Therefore, we will again rely on numerics to show some qualitative features, which will strengthen the results from the last section, and again hint toward section 5 .

Up to a $t$-dependent factor, the scalar product (4.20) between the two gauge-invariant coherent states $\Psi_{\left[h_{1}, h_{2}, h_{3}\right]}^{t}$ and $\Psi_{\left[g_{1}, g_{2}, g_{3}\right]}^{t}$ is given by

$$
\begin{aligned}
\left.\left\langle\Psi_{\left[h_{1}, h_{2}, h_{3}\right]}^{t}\right| \Psi_{\left[g_{1}, g_{2}, g_{3}\right]}^{t}\right] & \sim \int_{S U(2)^{2}} \mathrm{~d} \mu_{H}\left(k_{1}, k_{2}\right) \\
& \times \prod_{l=1}^{3} \sum_{n_{l} \in \mathbb{Z}} \frac{f_{l}\left(k_{1}, k_{2}\right)-2 \pi \mathrm{i} n_{l}}{\sinh \left(f_{l}\left(k_{1}, k_{2}\right)-2 \pi \mathrm{i} n_{l}\right)} \exp \left(\frac{\left(f_{l}\left(k_{1}, k_{2}\right)-2 \pi \mathrm{i} n_{l}\right)^{2}}{t}\right)
\end{aligned}
$$


with

$$
\cosh f_{l}\left(k_{1}, k_{2}\right)=\frac{1}{2} \operatorname{tr}\left(g_{l}^{\dagger} k_{1} h_{l} k_{2}^{-1}\right) .
$$

As in the case of the 2-flower graph, we start to gauge-fix the integrand by applying lemma 4.1. If we write

$$
h_{l}=\exp \left(\mathrm{i} \vec{\sigma} \cdot \vec{w}_{l}^{\prime}\right), \quad g_{l}=\exp \left(\mathrm{i} \vec{\sigma} \cdot \vec{z}_{l}^{\prime}\right)
$$

for $\vec{z}_{l}^{\prime}, \vec{w}_{l}^{\prime} \in \mathbb{C}^{3}$, and choose the $h_{l}, g_{l}$ to be well away from the negative Hermitean elements, we can choose the real part of the complex vectors to be in a ball around zero with radius $\pi$ : $\operatorname{Re} \vec{w}_{l}^{\prime}, \operatorname{Re} \vec{z}_{l}^{\prime} \in B_{\pi}(0)$, and thus we can neglect all terms in the infinite sum (4.37) apart from $n_{1}=n_{2}=n_{3}=0$. Changing the integration variables

$$
k_{1} \longmapsto\left(g_{1}^{\dagger}\right)^{-1} k_{1}, \quad k_{2} \longmapsto k_{2} h_{1}
$$

amounts to a change of the terms occurring in (4.37) as

$$
\begin{aligned}
& \frac{1}{2} \operatorname{tr}\left(g_{1}^{\dagger} k_{1} h_{1} k_{2}^{-1}\right) \longmapsto \frac{1}{2} \operatorname{tr}\left(k_{1} k_{2}^{-1}\right) \\
& \frac{1}{2} \operatorname{tr}\left(g_{2}^{\dagger} k_{1} h_{2} k_{2}^{-1}\right) \longmapsto \frac{1}{2} \operatorname{tr}\left(\tilde{g}_{2}^{\dagger} k_{1} \tilde{h}_{2} k_{2}^{-1}\right) \\
& \frac{1}{2} \operatorname{tr}\left(g_{3}^{\dagger} k_{1} h_{3} k_{2}^{-1}\right) \longmapsto \frac{1}{2} \operatorname{tr}\left(\tilde{g}_{3}^{\dagger} k_{1} \tilde{h}_{3} k_{2}^{-1}\right),
\end{aligned}
$$

with

$$
\tilde{g}_{l}=g_{1}^{-1} g_{l}, \quad \tilde{h}_{l}=h_{l} h_{1}^{-1} .
$$

Write

$$
\tilde{h}_{l}=\exp \left(\mathrm{i} \vec{\sigma} \cdot \vec{w}_{l}\right), \quad \tilde{g}_{l}=\exp \left(\mathrm{i} \vec{\sigma} \cdot \vec{z}_{l}\right)
$$

with vectors $\vec{w}_{l}, \vec{z}_{l} \in \mathbb{C}^{3}, l=2,3$. The only transformations of the integration variables $k_{1}, k_{2}$ that leave the form of the terms (4.41) invariant are simultaneously gauging

$$
k_{1,2} \longmapsto g^{\dagger} k_{1,2} h^{-1}
$$

for arbitrary $g, h \in S L(2, \mathbb{C})$. As with the coherent states on the 2-flower graph, this induces an $O(3, \mathbb{C})$ action on the vectors $\vec{w}_{l}, \vec{z}_{l}$, which can be used to gauge $\vec{w}_{2}$ and $\vec{z}_{3}$ to point into the 3-direction, and $\vec{w}_{3}, \vec{z}_{2}$ out of the 1-direction:

$$
\begin{aligned}
& \vec{w}_{2}=\left(\begin{array}{c}
0 \\
0 \\
w_{2}
\end{array}\right), \quad \vec{z}_{3}=\left(\begin{array}{c}
0 \\
0 \\
z_{3}
\end{array}\right) \\
& \vec{w}_{3}=\left(\begin{array}{c}
0 \\
w_{3} \sin \chi \\
w_{3} \cos \chi
\end{array}\right), \quad \vec{z}_{2}=\left(\begin{array}{c}
0 \\
z_{2} \sin \theta \\
z_{2} \cos \theta
\end{array}\right) .
\end{aligned}
$$

Note that the gauge-invariant information for gauge-invariant states on the 3-bridge graph are the same as those on the 2-flower graph. This was to be expected, since both have the same first fundamental group. The gauge-invariant inner product, on the other hand, looks slightly different:

$\left\langle\Psi_{\left[h_{1}, h_{2}, h_{3}\right]}^{t} \mid \Psi_{\left[g_{1}, g_{2}, g_{3}\right]}^{t}\right\rangle \approx \int_{S U(2)^{2}} \mathrm{~d} \mu_{H}\left(k_{1}, k_{2}\right) \prod_{l=1}^{3} \frac{f_{l}\left(k_{1}, k_{2}\right)}{\sinh f_{l}\left(k_{1}, k_{2}\right)} \exp \left(\frac{f_{l}\left(k_{1}, k_{2}\right)^{2}}{t}\right)$

with

$$
\begin{aligned}
& \cosh f_{2}(k)=\cos z_{2} \cos \bar{w}_{2}+\sin z_{2} \sin \bar{w}_{2} \cos \left(\tilde{\theta}\left(k_{1}, k_{2}\right)\right) \\
& \cosh f_{3}(k)=\cos z_{3} \cos \bar{w}_{3}+\sin z_{3} \sin \bar{w}_{3} \cos \left(\overline{\tilde{\chi}}\left(k_{1}, k_{2}\right)\right),
\end{aligned}
$$




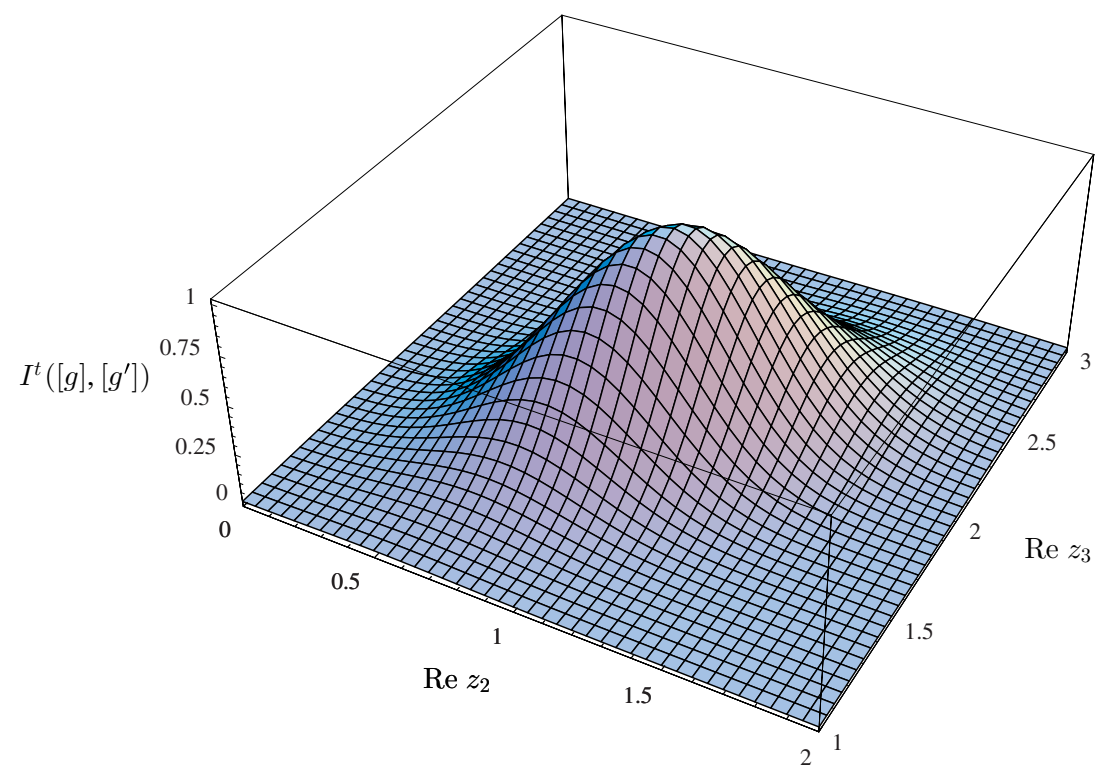

Figure 7. The overlap $I^{t}\left([g],\left[g^{\prime}\right]\right)$ between the two gauge-invariant coherent states $\Psi_{[g]}^{t}$ and $\Psi_{\left[g^{\prime}\right]}^{t}$ on the 3-bridge graph. One is labeled by the gauge-invariant data $w_{2}=1, w_{3}=2, \chi=0.3$. The other one is labeled by $\theta=0.3$, depending on $z_{2}, z_{3}$.

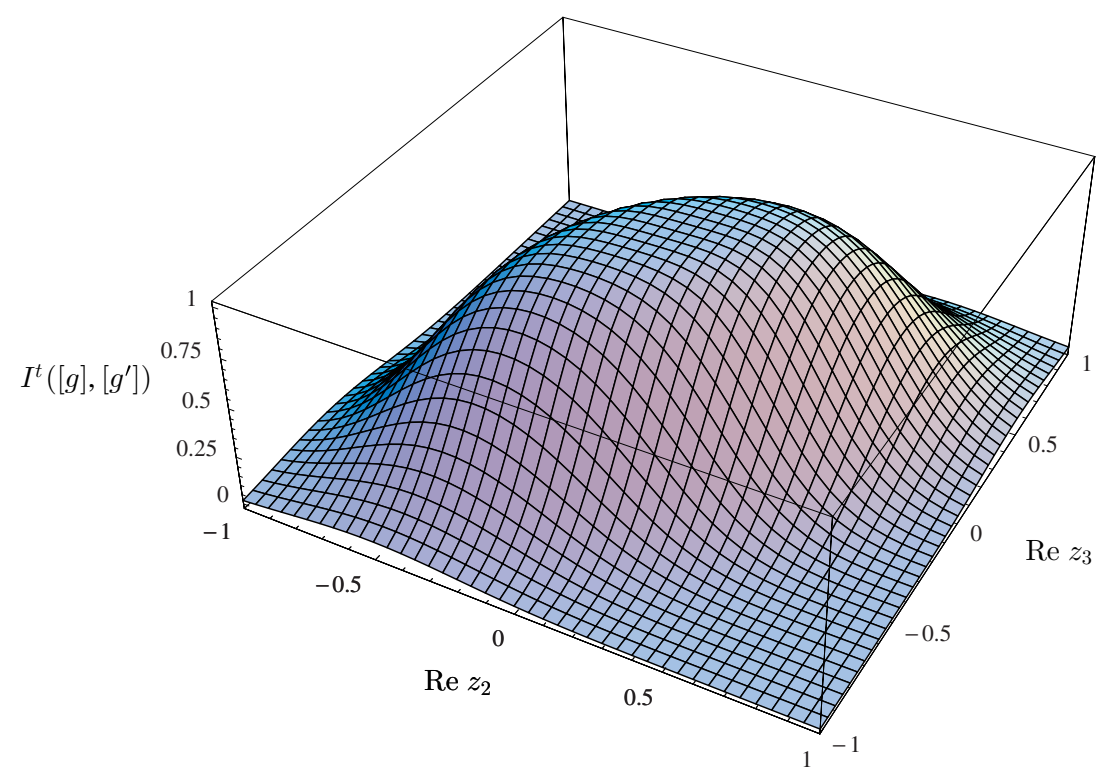

Figure 8. The overlap $I^{t}\left([g],\left[g^{\prime}\right]\right)$ between the two gauge-invariant coherent states $\Psi_{[g]}^{t}$ and $\Psi_{\left[g^{\prime}\right]}^{t}$ on the 3-bridge graph. One is labeled by the gauge-invariant data $w_{2}=0, w_{3}=0, \chi=0$, corresponding to $g_{l}=\mathbb{1} \in S L(2, \mathbb{C})$ for $l=1,2,3$. The other one is labeled at $\theta=0$, depending on $z_{2}, z_{3}$.

where $\tilde{\theta}\left(k_{1}, k_{2}\right)$ is the (complex) angle between $\pi_{1}\left(k_{1}\right) \overline{\bar{w}}_{2}$ and $\pi_{1}\left(k_{2}\right) \vec{z}_{2}$, and $\tilde{\chi}\left(k_{1}, k_{2}\right)$ is that between $\pi_{1}\left(k_{1}\right) \overrightarrow{\bar{w}}_{3}$ and $\pi_{1}\left(k_{2}\right) \vec{z}_{3}$. 


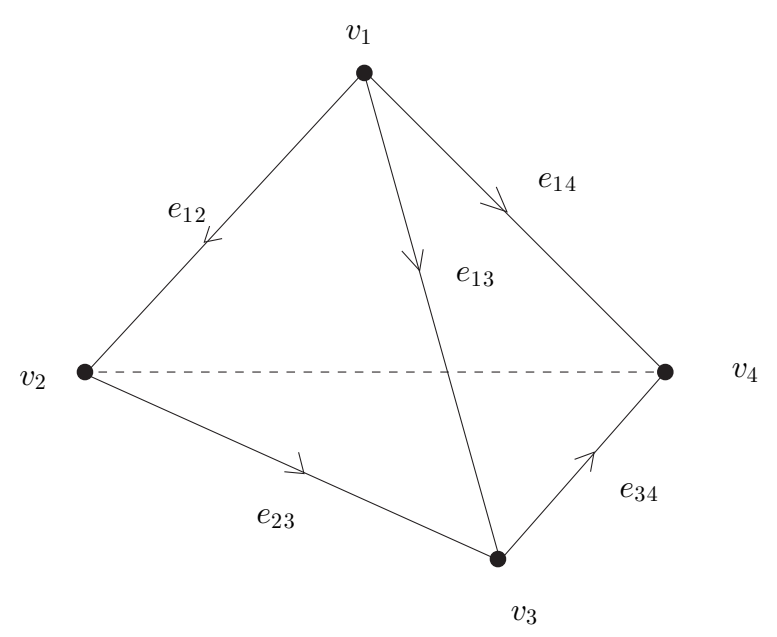

Figure 9. A tetrahedron with vertices $v_{I}, I=1, \ldots, 4$, and edges $e_{I J}, I<J$.

The gauge-fixed integrand can be evaluated numerically. We show the result for the two different gauge-invariant label sets.

The first plot, shown in figure 7, demonstrates again that the overlap is peaked at the point where both gauge-invariant data sets coincide.

The second plot, shown in figure 8, depicts the overlap of a state with that labeled by $[\mathbb{1}, \mathbb{1}, \mathbb{1}]$.

Again, one can see the plateau structure of the overlap. This feature was also visible with the 1-flower and the 2-flower graph, and shows that this phenomenon is not just a feature of the flower graphs. We will discuss this in section 5.

\subsection{The tetrahedron graph}

As well as the gauge-invariant overlap on the other graphs, the gauge-invariant overlap on the tetrahedron graph is completely out of reach of any analytical computations. Still, one can, in complete analogy to the case of the 2-flower or the 3-bridge graph, describe the gauge-invariant degrees of freedom in $S L(2, \mathbb{C})^{6}$ by a gauge-fixing procedure, and show numerically that the overlap of the gauge-invariant coherent states is peaked at the points where the two label sets coincide.

The tetrahedron is given by four vertices and six edges. The vertices are labeled by $v_{I}$ with $I=1, \ldots, 4$. There is an edge between any two vertices, $e_{I J}$, oriented from $v_{I}$ to $v_{J}$ for $I<J$ (see figure 9).

The gauge-invariant overlap will again be calculated from the inner product between the two gauge-invariant coherent states by (4.21). The gauge-invariant inner product is given by

$$
\begin{aligned}
\left\langle\Psi_{\left[g_{I J}\right]}^{t} \mid \Psi_{\left[h_{I J}\right]}^{t}\right\rangle & \sim \int_{S U(2)^{4}} \mathrm{~d} \mu_{H}^{\otimes 4}(k) \\
& \times\left[\prod_{I<J} \sum_{n_{I J} \in \mathbb{Z}} \frac{f_{I J}(k)-2 \pi \mathrm{i} n_{I J}}{\sinh \left(f_{I J}(k)-2 \pi \mathrm{i} n_{I J}\right)} \exp \left(\frac{\left(f_{I J}(k)-2 \pi \mathrm{i} n_{I J}\right)^{2}}{t}\right)\right]
\end{aligned}
$$




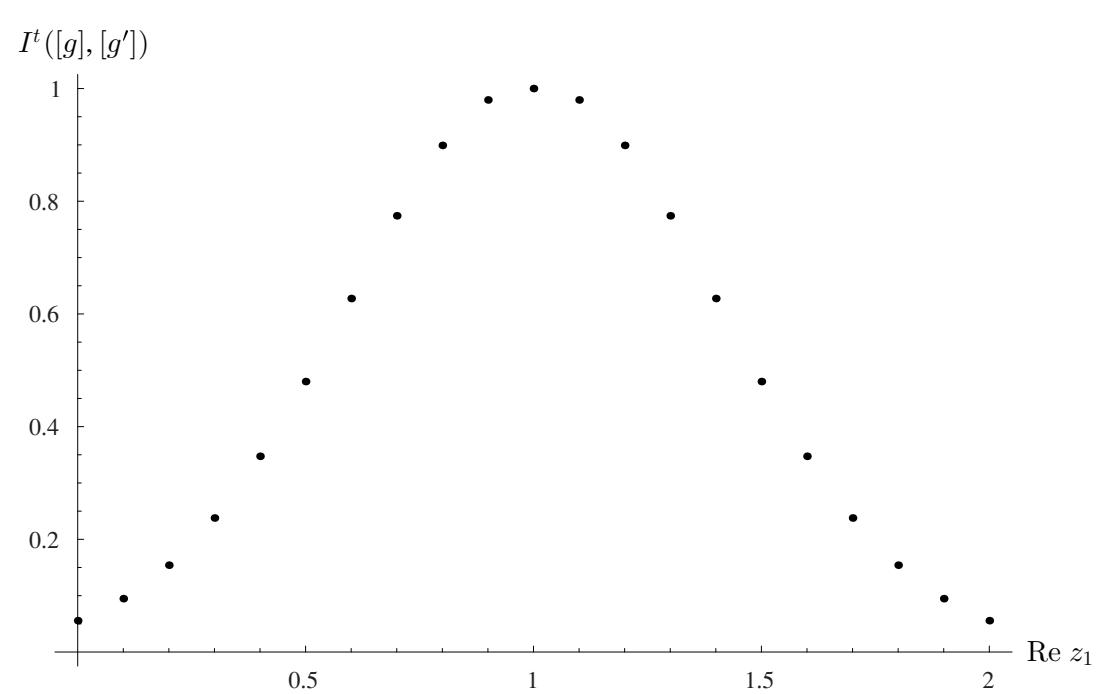

Figure 10. The overlap $I^{t}\left([g],\left[g^{\prime}\right]\right)$ between the gauge-invariant coherent states $\Psi_{[g]}^{t}$ and $\Psi_{\left[g^{\prime}\right]}^{t}$ labeled by gauge-invariant data $w_{1}=1, z_{2}=w_{2}=2, \chi=\theta=0.3, \vec{z}_{3}=\vec{w}_{3}=(1,1,1)$, depending on $z_{1}$.

with

$$
\cosh f_{I J}(k)=\frac{1}{2} \operatorname{tr}\left(g_{I J}^{\dagger} k_{I} h_{I J} k_{J}^{-1}\right) .
$$

A crucial point in the gauge-fixing procedure used to extract the gauge-invariant degrees of freedom is the use of a maximal tree $\tau$ in the tetrahedron graph $\gamma$. From usual lattice gauge theory, one knows that by successive gauging one can gauge a distribution of group elements along the edges of $\gamma$ such that there is one along each edge of $\tau$. The resulting distributions of elements among the (three) leaves of $\gamma$, i.e. the edges not belonging to $\tau$, contain (modulo global conjugation) the gauge-invariant information of the distribution of elements.

The remaining global conjugation freedom in $g_{k}=\mathrm{e}^{\mathrm{i} \vec{z}_{k} \cdot \vec{\sigma}} \in S L(2, \mathbb{C})$, with $\vec{z}_{k} \in \mathbb{C}^{3}$, corresponds to a similar $O(3, \mathbb{C})$ rotation of the $\vec{z}_{k}$. This rotation can-exactly as in the previous examples-be used to rotate one of the vectors into the 3-direction, another into the 2-3-plane, while the third vector is fixed then. The remaining degrees of freedom are then two complex lengths: a complex angle and a complex 3 -component vector. These six parameters, i.e. $z_{1}, z_{2}, \theta, \vec{z}_{3}$, determine the gauge-invariant data set, which corresponds to the geometry of a tetrahedron in $S L(2, \mathbb{C})$.

Unfortunately, since the integral (4.46) ranges over $S U(2)^{4}$ which is 12-dimensional, the numerical integration becomes quite involved. In particular, to compute overlaps with a sufficient precision is very time consuming. Still, we were able to produce some integrals, which show the peakedness of the overlap.

As one can see in figure 10 , the overlap is Gaussian peaked at the point $z_{1}=1$, i.e. where the two label sets coincide.

\section{Gauge-invariant coherent states for $G=S U(2)$ : general properties}

In the last section, we have investigated the gauge-invariant coherent states for the gauge group $S U$ (2) analytically and numerically for some simple examples. Unfortunately, the formula 
for the inner product between these states is too complicated in order to prove the peakedness properties of the overlap for arbitrary graphs.

Still, in the following sections we will investigate some properties of the gauge-invariant states on arbitrary graphs. In particular, we will be able, by an appropriate gauge-fixing procedure, to connect the inner product between two gauge-invariant coherent states on an arbitrary graph with $E$ edges and $V$ vertices to the inner product between gauge-invariant coherent states on an $(E-V+1)$-flower graph.

The same procedure is, in principle, also possible for the case of $G=U(1)$, which leads to a gauge-invariant coherent state on a flower graph, which can be, due to the Abelianess of the gauge group, explicitly written down. Remember that for Abelian gauge groups, all functions on flower graphs are automatically gauge invariant. This is, of course, no longer true for non-Abelian gauge groups, so relating the gauge-invariant coherent states on arbitrary graphs to gauge-invariant coherent states on flower graphs is the best one can do.

Still, this formula will allow us to prove a theorem about the peakedness properties of states labeled by $[\mathbb{1}, \ldots, \mathbb{1}]$. In the last section, we have already seen that the overlap between two states, one of them being labeled by $[\mathbb{1}, \ldots, \mathbb{1}]$, does not behave like a Gaussian. Rather, the profile of the overlap looks like a $\mathrm{e}^{-x^{4}}$ curve. In the following, we will prove this for arbitrary graphs.

We will start with a theorem about the peakedness of $\Psi_{[\mathbb{1}, \ldots, \mathbb{1}]}^{t}$ on flower graphs. Then, we will derive a formula relating the inner products of gauge-invariant coherent states on arbitrary graphs to those on flower graphs. This will ultimately enable us to formulate a corresponding theorem about the peakedness properties of $\Psi_{[\mathbb{1}, \ldots, 1]}^{t}$ on arbitrary graphs.

\subsection{Peakedness of $\Psi_{[\mathbb{1}, \ldots, \mathbb{1}]}^{t}$ on E-flower graphs}

In the previous sections, we have seen that the peakedness of the state $\Psi_{[\mathbb{1}, \ldots, \mathbb{1}]}^{t}$, i.e. the gaugeinvariant state labeled by the equivalence class of $(\mathbb{1}, \ldots, \mathbb{1}) \in S L(2, \mathbb{C})^{E}$, is qualitatively different than the peakedness of states that are labeled by generic elements. In particular, the overlap (4.29) on the 1-flower graph

$$
z \longmapsto \frac{\left|\left\langle\Psi_{[\mathbb{1}]}^{t} \mid \Psi_{\cos z}^{t}\right\rangle\right|^{2}}{\left\|\Psi_{\cos z}^{t}\right\|^{2}\left\|\Psi_{[\mathbb{1}]}^{t}\right\|^{2}}=\frac{2\left|z^{2}\right| / t}{\sinh \left(2|z|^{2} / t\right)}\left(1+O\left(t^{\infty}\right)\right)
$$

behaves like $\sim 1-\frac{2}{3 t^{2}}|z|^{4} \sim \exp \left(-2|z|^{4} / 3 t^{2}\right)$, as $z \rightarrow 0$, rather than a Gaussian. The plots of overlaps on the 2-flower graph support the conjecture that this is a general feature of states on $E$-flowers peaked at the equivalence class of $(\mathbb{1}, \ldots, \mathbb{1}) \in S L(2, \mathbb{C})^{E}$.

This conjecture is in fact true, as we will show now. Despite the notoriously complicated structure of the overlap, in this particular case we are able to perform the gauge integrals in the limit of the labelings being close to $(\mathbb{1}, \ldots, \mathbb{1})$.

Theorem 5.1. Let $\gamma$ be the E-flower graph, i.e. the graph with one vertex and E edges all starting and ending at that vertex. Define $h\left(\vec{z}_{j}\right) \in S L(2, \mathbb{C})^{E}$ by $h_{j}=\exp \left(i \vec{\sigma} \cdot \vec{z}_{j}\right) \in$ $S L(2, \mathbb{C}), j=1, \ldots, E$. Then one has

$$
\frac{\mid\left\langle\Psi_{[\mathbb{1}, \ldots, \mathbb{1}]}^{t}\left|\Psi_{\left[h\left(\vec{z}_{j}\right)\right]}^{t}\right|^{2}\right.}{\left\|\Psi_{[\mathbb{1}, \ldots, \mathbb{1}]}^{t}\right\|^{2}\left\|\Psi_{\left[h\left(\vec{z}_{j}\right)\right]}^{t}\right\|^{2}}=1-O\left(\left\|\vec{z}_{j}\right\|^{4}\right)
$$

Proof. Of course, $h\left(\vec{z}_{j} \equiv 0\right)=\mathbb{1}$, it is clear that the overlap between $\Psi_{[\mathbb{1}, \ldots, \mathbb{1}]}^{t}$ and $\Psi_{\left[h\left(\vec{z}_{j} \equiv 0\right)\right]}$ is equal to 1 . What we will do now to prove equation (5.1) is to expand the two inner products

$$
\left\langle\Psi_{[\mathbb{1}, \ldots, \mathbb{1}]}^{t} \mid \Psi_{\left[h\left(\vec{z}_{j}\right)\right]}^{t}\right\rangle \quad \text { and } \quad\left\|\Psi_{\left[h\left(\vec{z}_{j}\right)\right]}^{t}\right\|^{2}=\left\langle\Psi_{\left[h\left(\vec{z}_{j}\right)\right]}^{t} \mid \Psi_{\left[h\left(\vec{z}_{j}\right)\right]}^{t}\right\rangle
$$


into second order around $\vec{z}_{j}=0$. The odd orders all vanish, and we will be able to show that the second order of the $\vec{z}_{j}$ will cancel in the numerator and the denominator of (5.1), such that the first nontrivial order will be that of $\left\|\vec{z}_{j}\right\|^{4}$, obviously with a negative sign, since the overlap has to be at most 1 .

Remember that the overlap between two gauge-invariant coherent states on the $E$-flower graph, labeled by $[\tilde{h}]$ and $[h], h, \tilde{h} \in S L(2, \mathbb{C})^{E}$, is given by

$$
\begin{aligned}
\left\langle\Psi_{[\tilde{h}]}^{t} \mid \Psi_{[h]}^{t}\right\rangle= & \int_{S U(2)} \mathrm{d} \mu_{H}(k) \sum_{n_{1}, \ldots n_{E} \in \mathbb{Z}} \prod_{j=1}^{E}\left[\frac{f_{j}(k)-2 \pi \mathrm{i} n_{j}}{\sinh \left(f_{j}(k)-2 \pi \mathrm{i} n_{j}\right)}\right] \\
& \times \exp \left(\sum_{j=1}^{E} \frac{\left(f_{j}(k)-2 \pi \mathrm{i} n_{j}\right)^{2}}{t}\right),
\end{aligned}
$$

where

$$
\cosh f_{j}(k)=\frac{1}{2} \operatorname{tr}\left(\tilde{h}_{j}^{\dagger} k h_{j} k^{-1}\right) .
$$

We calculate the inner product between the two gauge-invariant coherent states on the $E$-flower graph, labeled by $h\left(\vec{w}_{j}\right)$ and $h\left(\vec{z}_{j}\right)$, expanding the expression in quadratic powers of the $\vec{z}_{j}$ and $\vec{w}_{j}$. The two contributions (5.2) are then just obtained by setting $\vec{w}_{j} \equiv 0$ and $\vec{w}_{j}=-i \overrightarrow{\bar{z}}_{j}$, respectively.

We write

$$
k=\exp (\mathrm{i} \vec{\sigma} \cdot \vec{\phi})=\cos \phi+\mathrm{i} \frac{\sin \phi}{\phi} \vec{\sigma} \cdot \vec{\phi}
$$

where, as usual,

$$
\phi:=\|\vec{\phi}\|=\sqrt{\left(\phi^{1}\right)^{2}+\left(\phi^{2}\right)^{2}+\left(\phi^{3}\right)^{2}} .
$$

Additionally, we get

$$
h\left(\vec{z}_{j}\right)=\exp \left(\mathrm{i} \vec{\sigma} \cdot \vec{z}_{j}\right)=\cos z_{j}+\mathrm{i} \frac{\sin z_{j}}{z_{j}} \vec{\sigma} \cdot \vec{z}_{j} \approx\left(1-\frac{1}{2} z_{j}^{2}\right)+\mathrm{i} \vec{\sigma} \cdot z_{j},
$$

again with

$$
z_{j}^{2}:=\vec{z}_{j} \cdot \vec{z}_{j}=\left(z_{j}^{1}\right)^{2}+\left(z_{j}^{2}\right)^{2}+\left(z_{j}^{3}\right)^{2},
$$

and according expressions for $h\left(\vec{w}_{j}\right)$. With these expressions, we get

$$
\begin{aligned}
\cosh f_{j}(k) \approx & \frac{1}{2} \operatorname{tr}\left[\left(1-\frac{1}{2} w_{j}^{2}+\mathrm{i} \vec{\sigma} \cdot \vec{w}_{j}\right)\left(\cos \phi+\mathrm{i} \frac{\sin \phi}{\phi} \vec{\sigma} \cdot \vec{\phi}\right)\right. \\
& \left.\times\left(1-\frac{1}{2} z_{j}^{2}+\mathrm{i} \vec{\sigma} \cdot \vec{z}_{j}\right)\left(\cos \phi-\mathrm{i} \frac{\sin \phi}{\phi} \vec{\sigma} \cdot \vec{\phi}\right)\right] \\
\approx & 1-\frac{z_{j}^{2}+w_{j}^{2}}{2}+2 \cos \phi \frac{\sin \phi}{\phi} \vec{\phi} \cdot\left(\vec{z}_{j} \times \vec{w}_{j}\right) \\
& +\left(\sin ^{2} \phi-\cos ^{2} \phi\right) \vec{w}_{j} \cdot \vec{z}_{j}-2 \frac{\sin ^{2} \phi}{\phi^{2}}\left(\vec{\phi} \cdot \vec{w}_{j}\right)\left(\vec{\phi} \cdot \vec{z}_{j}\right) \\
= & : 1+I_{j}\left(\vec{\phi}, \vec{z}_{j}, \vec{w}_{j}\right) .
\end{aligned}
$$

Here, the algebraic relations $\sigma_{I} \sigma_{J}=\delta_{I J}+\mathrm{i} \varepsilon_{I J K} \sigma_{K}$ and $\operatorname{tr} \sigma_{I}=0$ have been used; furthermore, only terms of the form $z^{2}, z w, w^{2}$ have been kept. Before we continue to calculate, it pays to 
think about which terms will play a role at all. First note that, since $h_{j}\left(\vec{w}_{j}\right)$ and $h_{j}\left(\vec{z}_{j}\right)$ are both close to $\mathbb{1} \in S L(2, \mathbb{C})$, they are also close to each other; hence, also $h_{j}\left(\vec{w}_{j}\right)^{c}$ and $h_{j}\left(\vec{z}_{j}\right)$ are close to each other. Thus, the main contribution of the infinite sums in (5.3) will come from the geodesics going directly from $h_{j}\left(\vec{w}_{j}\right)^{c}$ to $h_{j}\left(\vec{z}_{j}\right)$ rather than the longer ones. Technically, this means that only the term with $n_{1}=\ldots=n_{E}=0$ will contribute significantly to the integral. All other terms will be of order $O\left(t^{\infty}\right)$ compared to it.

Secondly, we note that $z \rightarrow(\operatorname{arccosh}(1+z))^{2} \approx 2 z+O\left(z^{4}\right)$ and $z / \sinh z \approx$ $1-z^{2} / 6+O\left(z^{4}\right)$. As $I_{j}\left(\vec{\phi}, \vec{z}_{j}, \vec{w}_{j}\right) \rightarrow 0$ as $\vec{z}_{j}, \vec{w}_{j} \rightarrow 0$, we can expand the terms in the exponential and the $z / \sinh z$ function, as well as the exponential itself into quadratic orders of $\vec{z}_{j}, \vec{w}_{j}$, and get

$$
\begin{aligned}
\left\langle\Psi_{\left[h\left(\vec{w}_{j}\right)\right]}^{t} \mid \Psi_{\left[h\left(\vec{z}_{j}\right)\right]}^{t}\right\rangle \approx & \int_{B_{\pi}(0)}\left[\frac{\sin ^{2} \phi}{\phi^{2}} \mathrm{~d}^{3} \phi\right]\left(1-\frac{\sum_{j=1}^{E} 2 I_{j}\left(\vec{\phi}, \vec{z}_{j}, \vec{w}_{j}\right)}{6}\right) \\
& \times\left(1+\frac{\sum_{j=1}^{E} 2 I_{j}\left(\vec{\phi}, \vec{z}_{j}, \vec{w}_{j}\right)}{t}\right) \\
& \approx \int_{B_{\pi}(0)}\left[\frac{\sin ^{2} \phi}{\phi^{2}} \mathrm{~d}^{3} \phi\right]\left(1+2\left(1-\frac{t}{6}\right) \sum_{j=1}^{E} \frac{I_{j}\left(\vec{\phi}, \vec{z}_{j}, \vec{w}_{j}\right)}{t}\right),
\end{aligned}
$$

where

$$
\int_{S U(2)} \mathrm{d} \mu_{H}(k) f(k)=\int_{B_{\pi}(0)} \frac{\sin ^{2}\|\vec{\phi}\|}{\|\vec{\phi}\|^{2}} \mathrm{~d}^{3} \phi f(\exp (\mathrm{i} \vec{\sigma} \cdot \vec{\phi}))
$$

has been used.

We immediately note the following interesting feature of the integral (5.6): it is linear in all the $I_{j}$ terms. Thus, we can perform the integration over each $I_{j}$, moreover, over each term in the $I_{j}$, separately. This huge simplification is due to the fact that $(\mathbb{1}, \ldots, \mathbb{1}) \in S L(2, \mathbb{C})^{E}$ is in fact a fixed point under the action of the gauge group, and for small $\vec{w}_{j}, \vec{z}_{j}$, the orbit always stays close to $(\mathbb{1}, \ldots, \mathbb{1})$.

So we only have to compute the integrals over $I_{j}$. In particular, writing

$$
\vec{\phi}=\phi\left(\begin{array}{c}
\cos \varphi \sin \vartheta \\
\sin \varphi \sin \vartheta \\
\cos \vartheta
\end{array}\right)
$$

it follows that

$$
\begin{aligned}
\int_{B_{\pi}(0)} \frac{\sin ^{2} \phi}{\phi^{2}} \mathrm{~d}^{3} \phi & =\frac{1}{2 \pi^{2}} \int_{0}^{\pi} \sin ^{2} \phi \mathrm{d} \phi \int_{S^{2}} \mathrm{~d} \Omega(\vartheta, \varphi) \\
& =\frac{1}{2 \pi^{2}} \int_{0}^{\pi} \sin ^{2} \phi \mathrm{d} \phi \int_{0}^{\pi} \sin \vartheta \mathrm{d} \vartheta \int_{0}^{2 \pi} \mathrm{d} \varphi
\end{aligned}
$$

and with this one can calculate

$$
\begin{aligned}
\int_{B_{\pi}(0)} \frac{\sin ^{2} \phi}{\phi^{2}} \mathrm{~d}^{3} \phi I_{j}\left(\vec{\phi}, \vec{w}_{j}, \vec{z}_{j}\right)=-\frac{z_{j}^{2}+w_{j}^{2}}{2} \\
+\frac{1}{\pi^{2}} \int_{0}^{\pi} \mathrm{d} \phi \sin ^{3} \phi \cos \phi \int_{S^{2}} \mathrm{~d} \Omega(\vartheta, \varphi)\left(\begin{array}{c}
\cos \varphi \sin \vartheta \\
\sin \varphi \sin \vartheta \\
\cos \vartheta
\end{array}\right) \cdot\left(\vec{z}_{j} \times \vec{w}_{j}\right)
\end{aligned}
$$




$$
\begin{aligned}
& +\frac{\vec{w}_{j} \cdot \vec{z}_{j}}{2 \pi^{2}} \int_{0}^{\pi} \mathrm{d} \phi \sin ^{2} \phi\left(\sin ^{2} \phi-\cos ^{2} \phi\right) \int_{S^{2}} \mathrm{~d} \Omega(\vartheta, \varphi) \\
& -\frac{1}{\pi^{2}} \int_{0}^{\pi} \mathrm{d} \phi \sin ^{4} \phi \int_{S^{2}} \mathrm{~d} \Omega(\vartheta, \varphi)\left[\left(\begin{array}{c}
\cos \varphi \sin \vartheta \\
\sin \varphi \sin \vartheta \\
\cos \vartheta
\end{array}\right) \cdot \vec{z}_{j}\right]\left[\left(\begin{array}{c}
\cos \varphi \sin \vartheta \\
\sin \varphi \sin \vartheta \\
\cos \vartheta
\end{array}\right) \cdot \vec{w}_{j}\right] .
\end{aligned}
$$

The first integral in this expression vanishes, simply because

$$
\int_{S^{2}} \mathrm{~d} \Omega(\vec{u}) \vec{u} \cdot \vec{x}=0
$$

for all $\vec{x} \in \mathbb{C}^{3}$. The second integral over $S^{2}$ is trivial, and the integral over $\phi$ is elementary. To evaluate the third integral we write

$$
\begin{aligned}
{\left[\left(\begin{array}{c}
\cos \varphi \sin \vartheta \\
\sin \varphi \sin \vartheta \\
\cos \vartheta
\end{array}\right) \cdot \vec{z}_{j}\right]\left[\left(\begin{array}{c}
\cos \varphi \sin \vartheta \\
\sin \varphi \sin \vartheta \\
\cos \vartheta
\end{array}\right) \cdot \vec{w}_{j}\right] } \\
=\left(z_{1} w_{1} \cos ^{2} \varphi \sin ^{2} \vartheta+\left(z_{1} w_{2}+z_{2} w_{1}\right) \cos \varphi \sin \varphi \sin \vartheta\right. \\
+w_{2} z_{2} \sin ^{2} \varphi \sin ^{2} \vartheta+\left(z_{1} w_{3}+z_{3} w_{1}\right) \cos \varphi \sin \vartheta \sin \varphi \\
\left.+\left(w_{2} z_{3}+w_{3} z_{2}\right) \sin \varphi \sin \vartheta \cos \vartheta+z_{3} w_{3} \cos ^{2} \vartheta\right)
\end{aligned}
$$

With elementary integrals, we get

$$
\begin{aligned}
\int_{B_{\pi}(0)} \frac{\sin ^{2} \phi}{\phi^{2}} \mathrm{~d}^{3} \phi I_{j}\left(\vec{\phi}, \vec{w}_{j}, \vec{z}_{j}\right) & =-\frac{z_{j}^{2}+w_{j}^{2}}{2}+\frac{1}{2} \vec{w}_{j} \cdot \vec{z}_{j}-\frac{1}{2} \vec{w}_{j} \cdot \vec{z}_{j} \\
& =-\frac{z_{j}^{2}+w_{j}^{2}}{2} .
\end{aligned}
$$

Thus, we arrive at the result

$$
\left\langle\Psi_{\left[h\left(\vec{w}_{j}\right)\right]}^{t} \mid \Psi_{\left[h\left(\vec{z}_{j}\right)\right]}^{t}\right\rangle=1-\left(1-\frac{t}{6}\right) \sum_{j=1}^{E} \frac{z_{j}^{2}+w_{j}^{2}}{t}+O\left(\left|z_{j}\right|^{4},\left|w_{j}\right|^{4}\right) .
$$

With this, the claim can be proven directly. Expanding the overlap between $\Psi_{[\mathbb{1}, \ldots \mathbb{1}]}^{t}$ and $\Psi_{\left[h\left(\vec{z}_{j}\right)\right]}^{t}$ into quadratic order reveals

$$
\begin{aligned}
\frac{\mid\left\langle\Psi_{[\mathbb{1}, \ldots, \mathbb{1}]}^{t}\left|\Psi_{\left[h\left(\vec{z}_{j}\right)\right]}^{t}\right|^{2}\right.}{\left\|\Psi_{[\mathbb{1}, \ldots, \mathbb{1}]}^{t}\right\|^{2}\left\|\Psi_{\left[h\left(\vec{z}_{j}\right)\right]}^{t}\right\|^{2}} & =1-\left(1-\frac{t}{6}\right) \sum_{j=1}^{E}\left(\frac{z_{j}^{2}}{t}+\frac{\bar{z}_{j}^{2}}{t}-\frac{z_{j}^{2}+\bar{z}_{j}^{2}}{t}\right)-O\left(\left|z_{j}\right|^{4}\right) \\
& =1-O\left(\left|z_{j}\right|^{4}\right) .
\end{aligned}
$$

This proves the claim of the theorem.

The previous theorem shows the effect that degenerate gauge orbits have on the peakedness properties of gauge-invariant coherent states. Although the states are still concentrated around the gauge orbits, the overlap function is no Gaussian anymore. Rather, the function decreases much slower, revealing a plateau around the phase-space point they are labeled with.

\subsection{Inner product of gauge-invariant coherent states}

In this section, we will relate the inner product of gauge-invariant coherent states on arbitrary graphs to the inner product of a corresponding state on a flower graph. A gauge-fixing procedure closely related to gauge-fixing in lattice gauge theory will be employed (see e.g. [17]). 
The inner product between two gauge-invariant coherent states on a graph $\gamma$ with $E$ edges and $V$ vertices is given by

$$
\begin{gathered}
\left\langle\Psi_{\left[g_{1}, \ldots, g_{E}\right]}^{t} \mid \Psi_{\left[h_{1}, \ldots, h_{E}\right]}^{t}\right\rangle=\frac{2^{E} \mathrm{e}^{E t / 4}}{\pi^{E}} \sqrt{\frac{\pi}{t}}^{3 E} \int_{S U(2)^{V}} \mathrm{~d} \mu_{H}\left(k_{1}, \ldots k_{V}\right) \\
\times \sum_{n_{1}, \ldots n_{E} \in \mathbb{Z}} \prod_{m=1}^{E} \frac{z_{m}-2 \pi \mathrm{i} n_{m}}{\sinh \left(z_{m}-2 \pi \mathrm{i} n_{m}\right)} \mathrm{e}^{\frac{(z m-2 \pi \mathrm{i} n m)^{2}}{t}}
\end{gathered}
$$

with

$$
\cosh z_{m}=\frac{1}{2} \operatorname{tr}\left(g_{m}^{\dagger} k_{b(m)} h_{m} k_{f(m)}^{-1}\right) .
$$

The key to the procedure is lemma 4.1. It enables us to shift an integration variable $k_{l}$ in (5.9) by elements in $S L(2, \mathbb{C})$ :

$$
k_{l} \longrightarrow G k_{l} H \quad \text { with arbitrary } H, G \in S L(2, \mathbb{C}) .
$$

Now choose a maximal tree $\tau$ in the graph $\gamma$. Remember that if $\gamma$ has $V$ vertices and $E$ edges, then $\tau$ has $V$ vertices and $V-1$ edges. Choose a vertex $\tilde{v}$. For each other vertex $v_{l} \in \gamma$ define

$$
G_{l}:=g_{e_{1}}^{ \pm 1} \cdot \ldots \cdot g_{e_{n}}^{ \pm 1} \quad H_{l}:=h_{e_{1}}^{ \pm 1} \cdot \ldots \cdot h_{e_{n}}^{ \pm 1} .
$$

Here, the edges $e_{1}, e_{2}, \ldots, e_{n}$ are the edges that one needs to go in the maximal tree $\tau$ from $v_{l}$ to $\tilde{v}$. This path is unique, as $\tau$ contains no loops. For each edge $e_{k}$ encountered, if the path from $v_{l}$ to $\tilde{v}$ goes against the orientation of the edge $e_{k}$, then take $g_{e_{k}}^{-1}$ and $h_{e_{k}}^{-1}$; if the path goes with the orientation of $e_{k}$, take $g_{e_{k}}$ and $h_{e_{k}}$. By this procedure, for each vertex $v_{l}$ two elements $G_{l}, H_{l}$ are defined (note that by definition the elements for the vertex $\tilde{v}$ are both $\mathbb{1} \in S L(2, \mathbb{C})$ ). Then, shift the integration in (5.9) by

$$
k_{l} \longrightarrow G_{l}^{\dagger} k_{l} H_{l}
$$

Let $e_{m}$ be an edge in $\tau$. The corresponding function (5.9) changes to

$$
\frac{1}{2} \operatorname{tr}\left(g_{m}^{\dagger} k_{b(m)} h_{m} k_{f(m)}^{-1}\right) \longrightarrow \frac{1}{2} \operatorname{tr}\left(k_{b(m)} k_{f(m)}^{-1}\right),
$$

whereas for some $e_{m}$ not being in $\tau$, the term (5.9) changes to

$$
\frac{1}{2} \operatorname{tr}\left(g_{m}^{\dagger} k_{b(m)} h_{m} k_{f(m)}^{-1}\right) \longrightarrow \frac{1}{2} \operatorname{tr}\left(\tilde{g}_{m}^{\dagger} k_{b(m)} \tilde{h}_{m} k_{f(m)}^{-1}\right)
$$

with $\tilde{g}_{m}$ and $\tilde{h}_{m}$ being the nontrivial products of various $g$ 's and $h$ 's, respectively. In particular, these products are given by

$$
\begin{aligned}
& \tilde{h}_{m}=h_{e_{1}}^{ \pm 1} \ldots h_{e_{n}}^{ \pm 1} h_{m} h_{e_{n+1}}^{ \pm 1} \ldots h_{e_{m}}^{ \pm 1} \\
& \tilde{g}_{m}=g_{e_{1}}^{ \pm 1} \ldots g_{e_{n}}^{ \pm 1} g_{m} g_{e_{n+1}}^{ \pm 1} \ldots g_{e_{m}}^{ \pm 1} .
\end{aligned}
$$

Here, the sequence of edges $e_{1}, \ldots, e_{n}, e_{m}, e_{n+1}, \ldots e_{N}$ is a loop in $\gamma$, starting at $\tilde{v}$, going to the beginning of the edge $e_{m}$ in $\tau$, going along $e_{m}$, and then going back to $\tilde{v}$, again along edges in $\tau$. Note that this path is unique. As usual, $h_{e_{l}}$ is taken if the path is going along the orientation of the edge, and $h_{e_{l}}^{-1}$ is taken if the path goes against the orientation of $e_{l}$. Similarly for $\tilde{g}$.

This gives two sets of $E-V+1$ elements in $S L(2, \mathbb{C})$, which are not gauge invariant, but behave quite simple under a global gauge transformation. It is quite easy to see that under some gauge transformation $k \equiv k_{v_{1}}=\cdots=k_{v_{V}}$ the new elements change as

$$
\tilde{h}_{e_{l}} \longrightarrow k \tilde{h}_{e_{l}} k^{-1} \text {. }
$$

A similar formula holds for $\tilde{g}$. With

$$
\arccos \frac{1}{2} \operatorname{tr}\left(k_{v}^{-1} k_{v^{\prime}}\right)=d\left(k_{v}, k_{v^{\prime}}\right),
$$


where $d$ denotes the geodesic distance on $S U$ (2) (for the shortest geodesic connecting $\left.k_{v}, k_{v^{\prime}} \in S U(2)\right)$, the above considerations give the following formula for the inner product between the two gauge-invariant coherent states:

$$
\begin{aligned}
\left\langle\Psi_{\left[g_{1}, \ldots, g_{E}\right]}^{t} \mid \Psi_{\left[h_{1}, \ldots, h_{E}\right]}^{t}\right\rangle=\frac{2^{E} \mathrm{e}^{E t / 4}}{\pi^{E}} \sqrt{\frac{\pi}{t}} \int_{S U(2)^{V}} \mathrm{~d} \mu_{H}\left(k_{1}, \ldots k_{V}\right) \\
\quad \times \sum_{n_{1}, \ldots n_{V-1} \in \mathbb{Z}} \prod_{m=1}^{V-1} \frac{d\left(k_{b(m)}, k_{f(m)}\right)-2 \pi n_{m}}{\sin d\left(k_{b\left(e_{m}\right)}, k_{f\left(e_{m}\right)}\right)} \exp \left[-\frac{\left(d\left(k_{b\left(e_{m}\right)}, k_{f\left(e_{m}\right)}\right)-2 \pi n_{m}\right)^{2}}{t}\right] \\
\quad \times \sum_{n_{V}, \ldots, n_{E} \in \mathbb{Z}} \prod_{m=V}^{E} \frac{z_{m}-2 \pi \mathrm{i} n_{m}}{\sinh \left(z_{m}-2 \pi \mathrm{i} n_{m}\right)} \exp \left[\frac{\left(z_{m}-2 \pi \mathrm{i} n_{m}\right)^{2}}{t}\right]
\end{aligned}
$$

with

$$
\cosh z_{m}=\frac{1}{2} \operatorname{tr}\left(\tilde{g}_{m}^{\dagger} k_{b(m)} \tilde{h}_{m} k_{f(m)}^{-1}\right)
$$

for $m=V, \ldots, E$. Note that this can be rewritten as

$$
\left\langle\Psi_{\left[g_{1}, \ldots, g_{E}\right]}^{t} \mid \Psi_{\left[h_{1}, \ldots, h_{E}\right]}^{t}\right\rangle=\left\langle\Psi_{\left[\mathbb{1}, \ldots, \mathbb{1}, \tilde{g}_{V}, \ldots \tilde{g}_{E}\right]}^{t} \mid \Psi_{\left[\mathbb{1}, \ldots, \mathbb{1}, \tilde{h}_{V}, \ldots, \tilde{h}_{E}\right]}^{t}\right\rangle .
$$

Thus, we have seen that the inner product between gauge-invariant coherent states labeled with arbitrary elements in $S L(2, \mathbb{C})$ is equal to the inner product of states where all labels corresponding to edges in a maximal tree $\tau$ are equal to $\mathbb{1} \in S L(2, \mathbb{C})$. This procedure can, in an obvious way, be carried over to the states themselves, showing that one can always gauge the labels corresponding to edges in $\tau$ to $\mathbb{1}$.

To make contact with the inner product of gauge-invariant coherent states on flower graphs, we make further modifications in formula (5.10). We now shift the integrations in (5.10) one after another.

First, order the set of vertices by the following method. Choose a vertex and call it $v_{1}$. For all $l=1, \ldots, V-1$, define $v_{l+1}$ such that the path from $v_{l+1}$ to $v_{1}$ through $\tau$ only passes the vertices $v_{1}, \ldots, v_{l}$, i.e. already ordered vertices. This gives, in the end, a (non-unique) numeration $v_{1}, \ldots, v_{V}$ of the vertices, which we will keep for the rest of this section.

The integration variables $k_{1}, \ldots, k_{V}$ appear in the integral (5.10) in the following combinations:

$$
\begin{aligned}
& k_{b(1)} k_{f(1)}^{-1} \\
& k_{b(2)} k_{f(2)}^{-1} \\
& \vdots \\
& k_{b(V-1)} k_{f(V-1)}^{-1} \\
& \tilde{g}_{V}^{\dagger} k_{b(V)} \tilde{h}_{V} k_{f(V)}^{-1} \\
& \vdots \\
& \tilde{g}_{E}^{\dagger} k_{b(E)} \tilde{h}_{E} k_{f(E)}^{-1} .
\end{aligned}
$$

We shift the elements $k_{1}, \ldots, k_{V}$ in ascending order, where the order of $k_{l}$ is determined by the numeration of vertices $v_{1}, \ldots, v_{V}$ defined above. First, we do not shift $k_{1}$. Second, we shift $k_{2}$ by

$$
k_{2} \longrightarrow k_{2} k_{1}
$$

We proceed by shifting the $k_{l}$ by

$$
k_{l} \longrightarrow k_{l} k_{a} k_{b} \ldots k_{1}=: \tilde{k}_{l}
$$


such that $v_{l} \rightarrow v_{a} \rightarrow v_{b} \rightarrow \cdots \rightarrow v_{1}$ is a path from the vertex $v_{l}$ to $v_{1}$ in the maximal tree $\tau$. So, as soon as a $k_{l}$ in (5.12) is replaced by the appropriate $\tilde{k}_{l}$, the single elements in it will not be altered by the following shifts, since the product of the $\tilde{k}_{l}$ only consists of elements $k_{1}, \ldots k_{l-1}$ that have already been shifted by the above procedure. It follows that, after this procedure, expressions (5.12), that ultimately appear in (5.10), will have changed to

$$
\begin{aligned}
& \tilde{k}_{b(1)} \tilde{k}_{f(1)}^{-1} \\
& \tilde{k}_{b(2)} \tilde{k}_{f(2)}^{-1} \\
& \vdots \\
& \tilde{k}_{b(V-1)} \tilde{k}_{f(V-1)}^{-1} \\
& \tilde{g}_{V}^{\dagger} \tilde{k}_{b(V)} \tilde{h}_{V} \tilde{k}_{f(V)}^{-1} \\
& \vdots \\
& \tilde{g}_{E}^{\dagger} \tilde{k}_{b(E)} \tilde{h}_{E} \tilde{k}_{f(E)}^{-1},
\end{aligned}
$$

where $\tilde{k}_{l}$, defined by (5.13), contain products of various $k_{l}$. But these products have the following properties.

First, for $1 \leqslant m \leqslant V-1$, we have that

$$
\tilde{k}_{b(m)} \tilde{k}_{f(m)}^{-1}=\left\{\begin{array}{lll}
k_{f(m)}^{-1} & \text { if } & e_{m} \text { is part of the path } v_{f(m)} \rightarrow v_{1} \\
k_{b(m)} & \text { if } & e_{m} \text { is part of the path } v_{b(m)} \rightarrow v_{1} .
\end{array}\right.
$$

One can easily see that the two cases are mutually excluding. From this we can also immediately deduce that in the first $V-1$ terms in (5.14), every $k_{l}$ from $k_{2}$ to $k_{V}$ appears exactly once, either as $k_{l}$ or as $k_{l}^{-1}$. Assume that a $k_{l}$ is appearing twice, either as $k_{l}$ or as $k_{l}^{-1}$ in the first $V-1$ terms in (5.14), say in terms $m$ and $m^{\prime}$. But from (5.15), $k_{l}$ appears only if the corresponding edge is part of the path from $v_{l}$ to $v_{1}$ in $\tau$. Having $k_{l}$ occurring in a place $m$ and $m^{\prime}$ means that the path from $v_{l}$ to $v_{1}$ in $\tau$ contains both $e_{m}$ and $e_{m^{\prime}}$. But, since $m \neq m^{\prime}$, this means that there are two different paths from $v_{l}$ to $v_{1}$ : one containing $e_{m}$, the other one containing $e_{m^{\prime}}$. But this is a contradiction, since the paths all lie entirely in $\tau$, which contains no loops; hence from any two vertices there is a unique path between them. This shows that in the first $V-1$ places in (5.14), every element $k_{l}$ appears at most once. And, since every $\tilde{k}_{l}$ ends with $k_{1}$, as one sees from (5.13), $k_{1}$ is the only one that is not appearing. So, (5.14) in fact looks like

$$
\begin{aligned}
& k_{2} \\
& k_{3} \\
& \vdots \\
& k_{V} \\
& \tilde{g}_{V}^{\dagger} \tilde{k}_{b(V)} \tilde{h}_{V} \tilde{k}_{f(V)}^{-1} \\
& \vdots \\
& \tilde{g}_{E}^{\dagger} \tilde{k}_{b(E)} \tilde{h}_{E} \tilde{k}_{f(E)}^{-1},
\end{aligned}
$$

where we have changed the order of the first $V-1$ terms, and have replaced all elements appearing in these terms at its inverse by the elements itself, which is allowed, since all terms in (5.16) appear inside a trace in (5.10), and $\operatorname{tr} k=\operatorname{tr} k^{-1}$ for all $k \in S U(2)$. 
The fact that in all $\tilde{k}_{l}, k_{1}$ appears only at the last position of the product (5.13) lets us rewrite the last $E-V+1$ terms in (5.16) as follows:

$$
\begin{aligned}
& k_{2} \\
& k_{3} \\
& \vdots \\
& k_{V} \\
& \left(\bar{k}_{V}^{-1} \tilde{g}_{V} \overline{\bar{k}}_{V}\right)^{\dagger} k_{1} \tilde{h}_{V} k_{1}^{-1} \\
& \vdots \\
& \left(\bar{k}_{E}^{-1} \tilde{g}_{E} \overline{\bar{k}}_{E}\right)^{\dagger} k_{1} \tilde{h}_{E} k_{1}^{-1},
\end{aligned}
$$

where

$$
\bar{k}_{m}:=k_{b(m)} k_{a} k_{b} \ldots k_{z} \quad \overline{\bar{k}}_{m}:=k_{f(m)} k_{a^{\prime}} k^{\prime} b \ldots k_{z^{\prime}},
$$

where the path $v_{b(m)} \rightarrow v_{a} \rightarrow v_{b} \rightarrow \cdots \rightarrow v_{z}$ is the path from $v_{b(f)}$ to $v_{1}$ (in $\tau$ ), excluding $v_{1}$ at the last position. Consequently, $v_{f(m)} \rightarrow v_{a^{\prime}} \rightarrow v_{b^{\prime}} \rightarrow \cdots \rightarrow v_{z^{\prime}}$ is the path from $v_{f(m)}$ to $v_{1}$ excluding $v_{1}$.

Having the integration variables shifted like this, we can finally recast (5.10) into the following form:

$$
\begin{aligned}
\left\langle\Psi_{\left[g_{1}, \ldots, g_{E}\right]}^{t} \mid \Psi_{\left[h_{1}, \ldots, h_{E}\right]}^{t}\right\rangle=\frac{2^{E} \mathrm{e}^{E t / 4}}{\pi^{E}} \sqrt{\frac{\pi}{t}}^{3 E} \int_{S U(2)^{V}} \mathrm{~d} \mu_{H}\left(k_{1}, \ldots k_{V}\right) \\
\quad \times \sum_{n_{2}, \ldots n_{V} \in \mathbb{Z}} \prod_{l=2}^{V} \frac{d\left(k_{l}\right)-2 \pi n_{l}}{\sin d\left(k_{l}\right)} \exp \left[-\frac{\left(d\left(k_{l}\right)-2 \pi n_{l}\right)^{2}}{t}\right] \\
\times \sum_{n_{V}, \ldots, n_{E} \in \mathbb{Z}} \prod_{m=V}^{E} \frac{z_{m}-2 \pi \mathrm{i} n_{m}}{\sinh \left(z_{m}-2 \pi \mathrm{i} n_{m}\right)} \exp \left[\frac{\left(z_{m}-2 \pi \mathrm{i} n_{m}\right)^{2}}{t}\right]
\end{aligned}
$$

with

$$
\cosh z_{m}=\frac{1}{2}\left(\left(\bar{k}_{m}^{-1} \tilde{g}_{m} \overline{\bar{k}}_{m}\right)^{\dagger} k_{1} \tilde{h}_{m} k_{1}^{-1}\right) .
$$

and $d(k):=d(\mathbb{1}, k)$ is the geodesic distance between $k$ and $\mathbb{1} \in S U(2)$.

But with this we immediately see that

$$
\begin{gathered}
\left\langle\Psi_{\left[g_{1}, \ldots, g_{E}\right]}^{t} \mid \Psi_{\left[h_{1}, \ldots, h_{E}\right]}^{t}\right\rangle=\frac{2^{V-1} \mathrm{e}^{(V-1) t / 4}}{\pi^{V-1}} \sqrt{\frac{\pi}{t}}{ }^{3 V-1} \int_{S U(2)^{V-1}} \mathrm{~d} \mu_{H}\left(k_{2}, \ldots k_{V}\right) \\
\quad \times \sum_{n_{2}, \ldots . n_{V} \in \mathbb{Z}} \prod_{l=2}^{V} \frac{d\left(k_{l}\right)-2 \pi n_{l}}{\sin d\left(k_{l}\right)} \exp \left[-\frac{\left(d\left(k_{l}\right)-2 \pi n_{l}\right)^{2}}{t}\right] \\
\times\left\langle\Psi_{\left[\bar{k}_{V}^{-1} \tilde{g}_{V} \bar{k}_{V}, \ldots, \bar{k}_{E}^{-1} \tilde{g}_{E} \overline{\bar{k}}_{E}\right]} \mid \Psi_{\left[\tilde{h}_{V}, \ldots, \tilde{h}_{E}\right]}^{t}\right\rangle,
\end{gathered}
$$

where the last term is the inner product between gauge-invariant coherent states on a $(E-V+1)$-flower graph, labeled with the (equivalence class of the) $E-V+1$ elements 
$\bar{k}_{V}^{-1} \tilde{g}_{V} \overline{\bar{k}}_{V}, \ldots, \bar{k}_{E}^{-1} \tilde{g}_{E} \overline{\bar{k}}_{E}$ and $\tilde{h}_{V}, \ldots, \tilde{h}_{E}$, respectively. Thus, the inner product between the gauge-invariant coherent states on arbitrary graphs can be related to the inner product of states on flower graphs.

The appearance of the Gaussian factors containing the geodesic distance of the elements $k_{2}, \ldots k_{V}$ to $\mathbb{1} \in S U(2)$ leads to the following idea concerning the behavior of the integral (5.20) in the limit of small $t$, which is the ultimate interest in LQG, where this $t$ is usually understood as some kind of semiclassicality scale, e.g. the ratio between Planck and classical scales. It is tempting to think of the following.

Consider the limit $t \rightarrow 0$. Then, the Gaussians in (5.20) will damp away all contributions to the integral not coming from the vicinity of $k_{2}=\cdots=k_{V}$. So, in the limit of small $t$ one might, at least asymptotically and up to a factor, say that

$$
\left\langle\Psi_{\left[g_{1}, \ldots, g_{E}\right]}^{t} \mid \Psi_{\left[h_{1}, \ldots, h_{E}\right]}^{t}\right\rangle \longrightarrow\left\langle\Psi_{\left[\tilde{g}_{V}, \ldots, \tilde{g}_{E}\right]}^{t} \mid \Psi_{\left[\tilde{h}_{V}, \ldots, \tilde{h}_{E}\right]}^{t}\right\rangle,
$$

where the first inner product is between gauge-invariant coherent states in (5.21) living on an arbitrary graph, while the second one is between states on a (corresponding) flower graph.

However, this deduction is, unfortunately, wrong. This is because the inner product between gauge-invariant coherent states on the $E-V+1$ flower graph contains the elements $k_{2}, \ldots k_{V}$ in the exponential with power $1 / t$. So, the reasoning that in the limit $t \rightarrow 0$ everything in the vicinity of $k_{2}=\ldots k_{V}=1$ is damped away is not correct, since the integrand itself could be an exponentially increasing function in the limit $t \rightarrow 0$ there. This can be best seen with an example. The above deduction states that, in the limit $t \rightarrow 0$, the main contribution to the integral,

$$
\frac{1}{\sqrt{\pi t}} \int_{\mathbb{R}} \mathrm{d} x \mathrm{e}^{-\frac{x^{2}}{t}} F_{t}(x),
$$

comes from $x \approx 0$, since everywhere else the integrand is exponentially small by the Gaussian. But, if for instance $F_{t}(x)$ is given by $\exp \left((x+z)^{2} / 2 t\right)$ for some complex number $z \in \mathbb{C}$, the main contribution to the integral

$$
\frac{1}{\sqrt{\pi t}} \int_{\mathbb{R}} \mathrm{d} x \mathrm{e}^{-\frac{x^{2}}{t}} \mathrm{e}^{\frac{(x+z)^{2}}{2 t}}
$$

does not come from $x \approx 0$, so one cannot assume

$$
\frac{1}{\sqrt{\pi t}} \int_{\mathbb{R}} \mathrm{d} x \mathrm{e}^{-\frac{x^{2}}{t}} \mathrm{e}^{\frac{(x+z)^{2}}{2 t}} \longrightarrow \mathrm{e}^{\frac{z^{2}}{2 t}},
$$

rather, the integral is equal to $\exp \left(z^{2} / t\right)$ instead. This is exactly the same situation as in (5.20) and is also the reason why the idea of (5.21) is a priori not right.

Still, in the following section we will, for arbitrary graphs and in the limit $t \rightarrow 0$, compute the overlap of a gauge-invariant coherent state labeled at $[\mathbb{1}, \ldots, \mathbb{1}]$ and another one, labeled at a point close to $[\mathbb{1}, \ldots, \mathbb{1}]$. By carefully performing the limits, we will be able to obtain a similar result as in the case of the flower graphs.

\subsection{Peakedness of $\Psi_{[\mathbb{1}, \ldots, \mathbb{1}]}^{t}$ on arbitrary graphs}

In this section, we will prove that for the small semiclassicality parameter $t$, the peakedness properties of the state $\Psi_{[\mathbb{1}, \ldots, \mathbb{1}]}^{t}$ are qualitatively different than the peakedness properties of generic gauge-invariant coherent states. We have already shown this for states on $E$-flower graphs, where this holds for arbitrary $t$. The strategy there was as follows. Our aim was to 
show that the overlap,

$$
\frac{\left|\left\langle\Psi_{[\mathbb{1}, \ldots, \mathbb{1}]}^{t} \mid \Psi_{\left[g_{1}, \ldots, g_{E}\right]}^{t}\right\rangle\right|^{2}}{\left\|\Psi_{[\mathbb{1}, \ldots, \mathbb{1}]}^{t}\right\|^{2}\left\|\Psi_{\left[g_{1}, \ldots, g_{E}\right]}\right\|^{2}},
$$

was, when expanding $g_{m}$ around the identity

$$
g_{m}=\exp \left(\overrightarrow{\mathrm{z}}_{m} \cdot \vec{\sigma}\right) \approx \mathbb{1}-\frac{z_{m}^{2}}{2}+\mathrm{i} \vec{z}_{m} \cdot \sigma
$$

not a Gaussian. In particular, we showed that

$$
(5.22)=1-O\left(\left\|\vec{z}_{m}\right\|^{4}\right),
$$

rather than of the form $1-O\left(\left\|\vec{z}_{m}\right\|^{2}\right)$, as would be expected from a Gaussian peak. We did this by noticing that the numerator and the denominator in (5.22) are even functions in the $\vec{z}_{m}$, so in the expansion only the even powers occur. Furthermore, we were able to show that the quadratic orders in the numerator and the denominator cancel, which shows that at most the quartic orders contribute to the overlap.

In all our calculations it was crucial that we were dealing with a state on a flower graph, since this allowed us to perform the gauge integral analytically for the second-order expansion. For arbitrary graphs, as we have seen in the last section, things are more difficult. Although we are able to relate the inner product of states on arbitrary graphs to those on flower graphs, this will not be enough to prove an equally strong result about the peakedness properties of $\Psi_{[1, \ldots, 1]}^{t}$ on arbitrary graphs. Still, the second order of the numerator and the denominator in (5.22) can be evaluated in the limit $t \rightarrow 0$. This shows that in the case of ultimate interest for LQG, where the semiclassicality parameter $t$ will be, depending on the application, about $t \approx 10^{-70}$, the states $\Psi_{[\mathbb{1}, \ldots, \mathbb{1}]}^{t}$ will have different peakedness properties than the states labeled by generic elements. As the plots for the 3-bridge suggest, this statement holds true also for arbitrary $t$; as in the case of the flower graphs, it is just that on arbitrary graphs the integrals become too complicated to evaluate, so the limit $t \rightarrow 0$ is what we have to live with.

We proceed along similar lines as for the flower graphs: we expand

$$
\left\langle\Psi_{\left[g_{1}, \ldots, g_{E}\right]}^{t} \mid \Psi_{\left[h_{1}, \ldots, h_{E}\right]}^{t}\right\rangle=\left\langle\Psi_{\left[\mathbb{1}, \ldots, \mathbb{1}, \tilde{g}_{V}, \ldots \tilde{g}_{E}\right]}^{t} \mid \Psi_{\left[\mathbb{1}, \ldots, \mathbb{1}, \tilde{h}_{V}, \ldots, \tilde{h}_{E}\right]}^{t}\right\rangle
$$

around $\tilde{g}_{m} \approx \tilde{h}_{m} \approx \mathbb{1}$, and consider the second-order contribution. We start with formula (5.10), and choose the elements $g_{1}, \ldots, g_{E}$ and $h_{1}, \ldots h_{E}$ all close to $\mathbb{1} \in S L(2, \mathbb{C})$. This, of course, implies that also the gauge-fixed quantities $\tilde{g}_{V}, \ldots \tilde{g}_{E}$ and $\tilde{h}_{V}, \ldots \tilde{h}_{E}$ are close to $\mathbb{1}$. In particular, we write

$$
\begin{aligned}
& \tilde{g}_{m}^{\dagger}=\exp \left(\mathrm{i} \vec{w}_{m} \cdot \vec{\sigma}\right) \approx 1-\frac{w_{m}^{2}}{2}+\mathrm{i} \vec{w}_{m} \cdot \vec{\sigma} \\
& \tilde{h}_{m}=\exp \left(\vec{i}_{m} \cdot \vec{\sigma}\right) \approx 1-\frac{z_{m}^{2}}{2}+\mathrm{i} \vec{z}_{m} \cdot \vec{\sigma}
\end{aligned}
$$

for some vectors $\vec{w}_{m}, \vec{z}_{m} \in \mathbb{C}^{3}$ being close to 0 , i.e. $\left\|\vec{w}_{m}\right\|,\left\|\vec{z}_{m}\right\| \ll 1$. This, of course, also implies that $w_{m}, z_{m}$ are complex numbers with small modulus:

$$
\left|w_{m}\right| \ll 1, \quad\left|z_{m}\right| \ll 1 \quad \text { for all } \quad m=V, \ldots, E .
$$

We now use expansion (5.24) to expand first

$$
\cosh z_{m}=\frac{1}{2} \operatorname{tr}\left(\tilde{g}_{m}^{\dagger} k_{b(m)} \tilde{h}_{m} k_{f(m)}^{-1}\right)
$$

and then ultimately the inner product between $\Psi_{\left[\mathbb{1}, \ldots, \mathbb{1}, \tilde{g}_{V}, \ldots, \tilde{g}_{E}\right]}^{t}$ and $\Psi_{\left[\mathbb{1}, \ldots, \mathbb{1}, \tilde{h}_{V}, \ldots, \tilde{h}_{E}\right]}^{t}$ into second order of $\vec{w}_{m}$ and $\vec{z}_{m}$. We will then see that in the limit of small $t$, the second-order term of the 
overlap between these two states vanishes. We start with expanding the cosh term into second order. With

$$
k_{b(m)}=\exp \left(\mathrm{i} \vec{\phi}_{m} \cdot \vec{\sigma}\right), \quad k_{f(m)}=\exp \left(\mathrm{i} \vec{\psi}_{m} \cdot \vec{\sigma}\right)
$$

we get

$$
\begin{aligned}
\frac{1}{2} \operatorname{tr}\left(\tilde{g}_{m}^{\dagger} k_{b(m)} \tilde{h}_{m} k_{f(m)}^{-1}\right) \approx \frac{1}{2} \operatorname{tr}\left(k_{b(m)} k_{f(m)}^{-1}\right)\left(1-\frac{w_{l}^{2}+z_{l}^{2}}{2}\right) \\
+\left(\cos \phi_{m} \frac{\sin \psi_{m}}{\psi_{m}} \vec{\psi}_{m}-\cos \psi_{m} \frac{\sin \phi_{m}}{\phi_{m}} \vec{\phi}_{m}\right) \cdot\left(\vec{z}_{m}+\vec{w}_{m}\right) \\
+\frac{\sin \phi_{m}}{\phi_{m}} \frac{\sin \psi_{m}}{\psi_{m}}\left(\vec{\phi}_{m} \times \vec{\psi}_{m}\right)\left(\vec{w}_{m}-\vec{z}_{m}\right) \\
+\left(\cos \phi_{m} \frac{\sin \psi_{m}}{\psi_{m}} \vec{\psi}_{m}+\cos \psi_{m} \frac{\sin \phi_{m}}{\phi_{m}} \vec{\phi}_{m}\right) \cdot\left(\vec{z}_{m} \times \vec{w}_{m}\right) \\
-\left(\cos \phi_{m} \cos \psi_{m}-\frac{\sin \phi_{m}}{\phi_{m}} \frac{\sin \psi_{m}}{\psi_{m}} \vec{\phi}_{m} \cdot \vec{\psi}_{m}\right) \vec{w}_{m} \cdot \vec{z}_{m} \\
\\
-\frac{\sin \phi_{m}}{\phi_{m}} \frac{\sin \psi_{m}}{\psi_{m}}\left(\vec{w}_{m} \cdot \vec{\psi}_{m}\right)\left(\vec{z}_{m} \cdot \vec{\phi}_{m}\right)
\end{aligned}
$$

Furthermore, if we expand the factors in (5.10), we get

$$
\begin{aligned}
& \frac{\operatorname{arccosh}(a+x)-2 \pi \mathrm{i} n}{\sinh \operatorname{arccosh} a+x} \mathrm{e}^{\frac{(\operatorname{arccosh}(a+x)-2 \pi \mathrm{in})^{2}}{t}} \\
& \approx \frac{\operatorname{arccosh}(a)-2 \pi \mathrm{i} n}{\sinh \operatorname{arccosh} a} \mathrm{e}^{\frac{(\operatorname{arccosh}(a)-2 \pi \mathrm{in})^{2}}{t}} \\
& \quad \times\left[1+\left(a+2 \frac{\sinh \operatorname{arccosh} a(\operatorname{arccosh} a-2 \pi n \mathrm{i})}{t}\right) x\right. \\
& +\frac{1}{2 t^{2} \sinh ^{4} \operatorname{arccosh} a}\left(-6 a \sqrt{a^{2}-1}(\operatorname{arccosh} a-2 \pi \mathrm{i} n) t\right. \\
& \left.\left.+4\left(a^{2}-1\right)(\operatorname{arccosh}(a)-2 \pi \mathrm{i} n)^{2}+t\left(t-6+2 a^{2}(3+t)\right)\right) x^{2}\right] .
\end{aligned}
$$

The following step would be to insert (5.25) into (5.26), then inserting this into (5.10) and performing the gauge integrals over $S U(2)^{V}$. While in the case for the $E$-flower graph the gauge integral turned out to be trivial, here this is no longer the case. On arbitrary graphs, this integral turns out to be too complicated to solve directly.

Still, we are able to perform this calculation in the limit $t \rightarrow 0$, which is of ultimate interest for LQG. In this limit the terms will simplify tremendously, so we will be able to produce the desired result.

First, we look at the zero-order term. This term amounts to setting $\tilde{g}_{m}=\tilde{h}_{m}=0$ in (5.10), hence the term of zero order is simply the norm of the coherent state $\Psi_{[\mathbb{1}, \ldots, \mathbb{1}]}^{t}$. Next, let us consider the terms of linear order in $\vec{z}_{m}, \vec{w}_{m}$. But from (5.10) we immediately see that the inner product (5.10) is an even function in these variables. Thus, the linear terms in the numerator and the denominator in (5.22) will vanish, as will all the odd order terms. So, the first terms that yield a nontrivial contribution are those that are of second order in the $\vec{w}_{m}, \vec{z}_{m}$. We will consider these now. 
Before we insert all the terms of the expansions made above into each other, it pays to look at how this integral behaves as $t \rightarrow 0$. The second order of (5.10) is of the form

$$
\begin{aligned}
\text { (2nd order) }= & \frac{2^{E} \mathrm{e}^{E t / 4}}{\pi^{E}} \sqrt{\frac{\pi}{t}} \int_{S U(2)^{V}}^{3 E} \mathrm{~d} \mu_{H}\left(k_{1}, \ldots, k_{V}\right) \\
& \times \sum_{n_{1}, \ldots n_{E} \in \mathbb{Z} m=1} \prod_{m}^{E} \frac{d\left(k_{b(m)}, k_{f(m)}\right)-2 \pi n_{m}}{\sin d\left(k_{b(m)}, k_{f(m)}\right)} \exp \left[-\frac{\left(d\left(k_{b(m)}, k_{f(m)}\right)-2 \pi n_{m}\right)^{2}}{t}\right] \\
& \times F_{t}\left(\vec{z}_{V}, \ldots, \vec{z}_{E}, \vec{w}_{V}, \ldots, \vec{w}_{E}, k_{1}, \ldots, k_{V}\right) .
\end{aligned}
$$

Here, the function $F_{t}$ captures the complicated expansion made above. Note however that $F_{t}$, although depending on $t$, does not diverge exponentially, as $t \rightarrow 0$, in contrast to the whole overlap in (5.10). There, the fact that the integrand is actually containing an exponential with $1 / t$-dependence, spoiled the possibility of using the method of stationary phase. If one does not want to compute the whole inner product, but only the second order of (5.10), we can now perform the limit $t \rightarrow 0$. Of course, since $F_{t}$ contains inverse powers of $t$, the limit of $F_{t}$ does not exist. But this could not have been expected, since the inner product between two coherent states is not a function of which the limit $t \rightarrow 0$ exists. But, as we will show now, the second derivative of (5.10) is of the form that it cancels in the numerator and the denominator in (5.22) asymptotically, as $t$ tends to 0 .

From the form (5.27) we immediately see that for small $t$, the integrand is exponentially damped in regions where not all of the $k_{1}, \ldots, k_{V}$ coincide. This is due to the fact that the integrand contains Gaussians in the geodesic distance between pairs of $k_{1}, \ldots, k_{V}$. Note that since the graph $\gamma$ is connected, the integrand is really concentrated around $k_{1}=\cdots=k_{V}$ for small $t$. From this we immediately conclude that, up to orders of $O\left(t^{\infty}\right)$, we can restrict our calculations to the summand with $n_{1}=\cdots n_{E}=0$. Then we see that the part of the integrand containing the exponentials

$$
\sqrt{\frac{\pi}{t}}^{3(V-1)} \prod_{m=1}^{E} \exp \left[-\frac{d\left(k_{b(m)}, k_{f(m)}\right)^{2}}{t}\right]
$$

effectively behaves as a delta function in the limit $t \rightarrow 0$, times a constant $C$, which is given by

$C=\lim _{t \rightarrow 0} \sqrt{\frac{\pi}{t}}^{3(V-1)} \int_{S U(2)^{V}} \mathrm{~d} \mu_{H}\left(k_{1}, \ldots, k_{V}\right) \prod_{m=1}^{E} \exp \left[-\frac{d\left(k_{b(m)}, k_{f(m)}\right)^{2}}{t}\right]$.

To compute $C$, we use the right invariance of the Haar measure and shift all integration variables $k_{l}$ other than $k_{1}$ by

$$
k_{l} \longrightarrow k_{l} k_{1} \text {. }
$$

We note that this leaves all terms unchanged in which $k_{1}$ does not appear, since the geodesic distance is invariant under right translation. In those terms, in which $k_{1}$ does appear, though, the transformation from the other element will effectively cancel it, which leaves us with

$$
C=\left.\lim _{t \rightarrow 0} \sqrt{\frac{\pi}{t}}^{3(V-1)} \int_{S U(2)^{V}} \mathrm{~d} \mu_{H}\left(k_{1}, \ldots, k_{V}\right) \prod_{m=1}^{E} \exp \left[-\frac{d\left(k_{b(m)}, k_{f(m)}\right)^{2}}{t}\right]\right|_{k_{1}=\mathbb{1}} .
$$

Note that now the main contribution to this integral comes from the vicinity of $k_{1}=k_{2}=$ $\ldots=k_{V}=\mathbb{1}$. Writing

$$
k_{l}=\exp \left(\overrightarrow{\mathrm{i}}_{l} \cdot \vec{\sigma}\right)
$$


we recast the Haar measure of $S U(2)$ into

$$
\int_{S U(2)} \mathrm{d} \mu_{H}(k) f(k)=\int_{B_{\pi}(0)} \mathrm{d}^{3} \phi \frac{\sin ^{2} \phi}{\phi^{2}} f(\exp (\mathrm{i} \vec{\phi} \cdot \vec{\sigma}))
$$

with $B_{\pi}(0)=\{\vec{\phi} \mid\|\vec{\phi}\|<\pi\}$. Now note that for $k_{l} \approx k_{l^{\prime}}$ we have

$$
\begin{aligned}
d\left(k_{l}, k_{l^{\prime}}\right)^{2} & =\arccos \left(\cos \phi_{l} \cos \phi_{l^{\prime}}-\frac{\sin \phi_{l}}{\phi_{l}} \frac{\sin \phi_{l^{\prime}}}{\phi_{l^{\prime}}} \vec{\phi}_{l} \cdot \vec{\phi}_{l^{\prime}}\right) \\
& =\left\|\vec{\phi}_{l}-\vec{\phi}_{l^{\prime}}\right\|^{2}+O\left(\left\|\vec{\phi}_{l}-\vec{\phi}_{l^{\prime}}\right\|^{4}\right),
\end{aligned}
$$

so, in the limit $t \rightarrow 0$ we can write

$$
\begin{aligned}
C & =\left.\lim _{t \rightarrow 0} \sqrt{\frac{\pi}{t}}^{3(V-1)} \int_{B_{\pi}(0)^{V}} \prod_{l=1}^{V}\left[\frac{\sin ^{2} \phi_{l}}{\phi_{l}^{2}} \mathrm{~d}^{3} \phi_{l}\right] \prod_{m=1}^{E} \exp \left[-\frac{\left\|\vec{\phi}_{b(m)}-\vec{\phi}_{f(m)}\right\|^{2}}{t}\right]\right|_{\vec{\phi}_{1}=0} \\
& =\left.\lim _{t \rightarrow 0} \sqrt{\frac{\pi}{t}}^{3(V-1)} \int_{B_{\pi}(0)^{V-1}} \prod_{l=2}^{V}\left[\frac{\sin ^{2} \phi_{l}}{\phi_{l}^{2}} \mathrm{~d}^{3} \phi_{l}\right] \prod_{m=1}^{E} \exp \left[-\frac{\left\|\vec{\phi}_{b(m)}-\vec{\phi}_{f(m)}\right\|^{2}}{t}\right]\right|_{\vec{\phi}_{1}=0},
\end{aligned}
$$

since the integration over $\vec{\phi}_{1}$ is trivial and the Haar measure is normalized. Since we integrate a Gaussian that becomes more and more concentrated around $\vec{\phi}_{l}=0$ as $t \rightarrow 0$, we make an error of order $O\left(t^{\infty}\right)$ if we extend the integration range over all of $\mathbb{R}^{3(V-1)}$. In this limit, since

$$
\left.\frac{\sin ^{2} \phi_{l}}{\phi_{l}^{2}}\right|_{\vec{\phi}=0}=1
$$

we have

$C=\lim _{t \rightarrow 0} \sqrt{\frac{\pi}{t}}^{3(V-1)} \int_{\mathbb{R}^{3 V}} \mathrm{~d}^{3 V} \phi_{l} \exp \left[\sum_{m=1}^{E} \frac{\left\|\vec{\phi}_{b(m)}-\vec{\phi}_{f(m)}\right\|^{2}}{t}\right] \delta^{(3)}\left(\vec{\phi}_{1}\right)$

with $\delta^{(3)}$ being the three-dimensional delta distribution. We have already encountered a similar integral in the case of $G=U(1)$. There, this integral could be solved by introducing the incidence matrix $\lambda \in \operatorname{Mat}(E \times V, \mathbb{Z})$, indicating which edges and vertices of the graph $\gamma$ are linked to each other.

We note that the exponential function in the integral is invariant under simultaneous shift of all integration variables by a constant vector:

$$
\vec{\phi}_{l} \longrightarrow \vec{\phi}_{l}+\vec{a}
$$

In this case, we can use a three-dimensional version of the lemma that has been used in the $U(1)$ case [1].

Lemma 5.1. Let $f: \mathbb{R}^{3 n} \rightarrow \mathbb{C}$ be a function with the symmetry

$$
f\left(\vec{\phi}_{1}+\vec{a}, \ldots, \vec{\phi}_{n}+\vec{a}\right)=f\left(\vec{\phi}_{1}, \ldots, \vec{\phi}_{n}\right) \quad \text { for all } \quad \vec{a} \in \mathbb{R}^{3}
$$

such that $\vec{\phi}_{2}, \ldots, \vec{\phi}_{n} \rightarrow f\left(0, \vec{\phi}_{2}, \ldots, \vec{\phi}_{n}\right)$ is integrable. Then

$$
\begin{aligned}
\int_{\mathbb{R}^{3(n-1)}} \mathrm{d}^{3} \phi_{2} & \cdots \mathrm{d}^{3} \phi_{n} f\left(0, \vec{\phi}_{2}, \vec{\phi}_{2}, \ldots, \vec{\phi}_{n}\right) \\
= & n^{3} \int_{\mathbb{R}^{3 n}} \mathrm{~d}^{3} \phi_{1} \cdots \mathrm{d}^{3} \phi_{n} \delta^{(3)}\left(\vec{\phi}_{1}+\cdots+\vec{\phi}_{n}\right) f\left(\vec{\phi}_{1}, \ldots, \vec{\phi}_{n}\right) .
\end{aligned}
$$

The proof of this lemma works completely analogous to that we have delivered in the case of the gauge-invariant coherent states with the gauge group $U(1)$. 
With these ingredients, we can write the constant $C$ as

$$
\begin{aligned}
C & =\lim _{t \rightarrow 0} V^{3} \sqrt{\frac{\pi}{t}}^{3(V-1)} \int_{\mathbb{R}^{3 V}} \mathrm{~d}^{3} \phi_{1} \ldots \mathrm{d}^{3} \phi_{V} \delta^{(3)}\left(\vec{\phi}_{1}+\cdots+\vec{\phi}_{V}\right) \exp \left[-\sum_{l, l^{\prime}=1}^{V} \frac{\lambda_{m l} \lambda_{m l^{\prime}} \vec{\phi}_{l} \cdot \vec{\phi}_{l^{\prime}}}{t}\right] \\
& =\sqrt{\pi}^{3(V-1)} V^{3} \int_{\mathbb{R}^{3 V}} \mathrm{~d}^{3} \phi_{1} \ldots \mathrm{d}^{3} \phi_{V} \delta^{(3)}\left(\vec{\phi}_{1}+\cdots+\vec{\phi}_{V}\right) \exp \left[-\sum_{l, l^{\prime}=1}^{V} \phi^{T} \Lambda \phi\right]
\end{aligned}
$$

by scaling the integration range. Here, $\phi$ stands for the collection $\phi_{l}^{I}$, where $l=1, \ldots, V$ go though the list of vertices, while $I=1,2,3$ denotes the component of $\vec{\phi}_{l}$.

Since the graph $\gamma$ we are considering is connected, Kirchhoff's theorem [16] tells us that the matrix

$$
\Lambda_{l l^{\prime}}^{I J}=\left(\lambda \lambda^{T}\right)_{l l^{\prime}} \delta^{I J}
$$

has three zero-eigenvectors, spanned by

$$
(\vec{a}, \ldots \vec{a}) \in \mathbb{R}^{3} \otimes \mathbb{R}^{V}
$$

for all $\vec{a} \in \mathbb{R}^{3}$. The three-dimensional delta function in (5.30) ensures that the integration ranges over the orthogonal complement of the zero space of $\Lambda$. But Kirchhoff's theorem tells us that all other eigenvalues $\mu_{2}, \ldots \mu_{V}$ of $\lambda \lambda^{T}$ are positive, and their product divided by $V$ gives

$$
G=\frac{1}{V} \prod_{l=2}^{V} \mu_{l}
$$

where $G$ is the number of different possible maximal trees in $\gamma$. If we know the eigenvalues of $\lambda \lambda^{T}$, we also know those of $\Lambda$, and can perform the integration (5.30), which only consists of multiple Gaussians. Finally, we arrive at

$$
C=\pi^{3(V-1)} \sqrt{\frac{V}{G}}^{3}
$$

So, in the limit $t \rightarrow 0$, we see that part of the integrand (5.27) behaves as a delta distribution. As already mentioned, $F_{t}$ does not converge for $t \rightarrow 0$, but from (5.25) and (5.26) we see that $F_{t}$ is a sum of terms, which are proportional to negative powers of $t$. So, although the integral (5.27) does not converge for $t \rightarrow 0$, we will compute it asymptotically, which will be enough to see that the second order of the numerator and the denominator in (5.22) cancel in the limit $t \rightarrow 0$.

With (5.31) and (5.28), we are able to evaluate (5.27) asymptotically:

(2nd order) $\sim \frac{2^{E} \mathrm{e}^{E t / 4} \pi^{2 E}}{\sqrt{\pi t}} \sqrt{\frac{V^{3(E-V+1)}}{G}} \int_{S U(2)} \mathrm{d} \mu_{H}(k) F_{t}\left(\vec{z}_{V}, \ldots, \vec{z}_{E}, \vec{w}_{V}, \ldots, \vec{w}_{E}, k, \ldots, k\right)$.

So, in the limit $t \rightarrow 0$ the integration over all variables $k_{1}, \ldots, k_{V}$ is restricted to the integration over the submanifold of all $k_{1}=\cdots=k_{V}=k$ being equal. On this set, however, expansions (5.25) and (5.26) simplify tremendously. These simplification amount to setting $\vec{\phi}_{l}=\vec{\psi}_{l}$ in (5.25) and setting $a=1$ (and $n=0$ ) in (5.26). With this, one can readily check that

(2nd order) $\sim-\left(1-\frac{t}{6}\right) \frac{2^{E} \mathrm{e}^{E t / 4} \pi^{2 E}}{\sqrt{\pi t}^{3(E-V+1)}} \sqrt{\frac{V}{G}} \sum_{m=V}^{E} \frac{z_{m}^{2}+w_{m}^{2}}{t}$, 
exactly as in the case for the flower graph. So, as has happened there, the quadratic orders in the numerator and the denominator of (5.22) cancel, and we arrive at

$$
\frac{\left|\left\langle\Psi_{[1, \ldots, \mathbb{1}]}^{t} \mid \Psi_{\left[g_{1}, \ldots, g_{E}\right]}^{t}\right\rangle\right|}{\left\|\Psi_{[1, \ldots, \mathbb{1}]}^{t}\right\|^{2}\left\|\Psi_{\left[g_{1}, \ldots, g_{E}\right]}\right\|^{2}} \longrightarrow 1-O\left(\left\|\vec{w}_{m}\right\|^{4}\right)
$$

Here, the arrow denotes that the quadratic order vanishes in the limit $t \rightarrow 0$, so that at most the quartic order remains. So, for small $t$, one can expect the gauge-invariant coherent state $\Psi_{[\mathbb{1}, \ldots, \mathbb{1}]}^{t}$ to be no Gaussian.

A couple of remarks are in order.

First, note that all of the above considerations could also have been carried out with any distribution of $\pm \mathbb{1}$ instead of just $\mathbb{1}$. In particular, all that we have derived above for $\Psi_{[\mathbb{1}, \ldots, \mathbb{1}]}^{t}$ is equally true for all $\Psi_{[ \pm \mathbb{1}, \ldots, \pm \mathbb{1}]}^{t}$. That is states that are labeled by points on which the gauge group $S U(2)^{V}$ acts trivial on $S L(2, \mathbb{C})^{E}$ in the case of the $E$-flower graph. For arbitrary graphs, the orbit of any distribution $\pm \mathbb{1}, \ldots, \pm \mathbb{1}$ along the leaves of a graph and $\mathbb{1}$ on the edges of the tree $\tau$ is left invariant by the gauge transformations $k_{1}=k_{2}=\cdots k_{V} \equiv k$, unlike generic points in $S L(2, \mathbb{C})^{E}$. So, the gauge orbit of $[ \pm \mathbb{1}, \ldots, \pm \mathbb{1}]$ has three dimensions less than orbits of generic points ${ }^{4}$. In particular, the gauge-invariant states have different peakedness properties when labeled at these points, where the gauge orbits do not have the full dimension. These points correspond to the singular points on the orbifold which consists of the gauge orbits.

Second, while the theorem about the qualitatively different peakedness behavior of $\Psi_{[ \pm \mathbb{1}, \ldots, \pm \mathbb{1}]}^{t}$ could be shown for finite $t$ in the case of the $E$-flower graph, for arbitrary graphs we could only establish this theorem in the limit $t \rightarrow 0$. One should be careful to note that this limit does not commute with the expansion of the overlap $I^{t}(\vec{w}, \vec{z})$ around the point $\vec{z}_{k}=\vec{w}_{k}$ (as one can most clearly see in the second order of the inner product (5.33), which does not converge for $t \rightarrow 0$ ). Clearly, in the limit $t \rightarrow 0$ the overlap converges pointwise to the function being equal to 1 on the point $\vec{z}_{k}=\vec{w}_{k}$ for all $k$, and being 0 elsewhere. This function clearly has no second derivative at $\vec{z}_{k}=\vec{w}_{k}$ in the function sense. On the other hand, we are interested in the asymptotic behavior of the second derivative of the overlap, as $t$ becomes small, but still finite. The order of first expanding the overlap into powers of $\left\|\vec{w}_{k}-\vec{z}_{k}\right\|$ and then showing that the quadratic term vanishes in the limit of $t \rightarrow 0$ is therefore justified.

Third, although we could show the vanishing of the second derivative of the overlap only in the limit $t \rightarrow 0$, we believe this theorem even to be true for arbitrary, finite $t$. There are two hints that support this conjecture: first, it is true for $E$-flower graphs. For arbitrary graphs it could not be shown due to the complicated form of the remaining integral (5.20), but this does not mean that it is not true. Rather, the degenerate points of the gauge orbits are present in the gauge orbit space for every graph, and are generic for non-Abelian gauge theories. So flower graphs do not seem to be special in this respect.

The second reason why we believe this to be true for arbitrary graphs is that the flatness of the overlap function could also be seen numerically for the 3-bridge graph, even for values of $t$ that are not incredibly tiny. So, we think that states labeled on gauge orbits degenerate to a point exhibit a qualitatively different peakedness behavior than states labeled on generic gauge orbits.

\footnotetext{
4 The only exception being the 1-flower graph, where, due to the Hopf fibration, the generic orbits are two dimensional,
} while the degenerate points $\pm \mathbb{1}$ are fixed points, i.e. have only two dimensions less than generic orbits. 


\section{Summary and conclusion}

\subsection{Summary of the work}

This work constitutes the second part of a pair of papers that investigate the gauge-invariant coherent states for LQG. The first paper investigated the simpler model of the Abelian gauge group $G=U(1)$, from which $G=U(1)^{3}$, which has been employed in LQG, can be immediately obtained. This paper considered the much more complicated, but also more realistic case of $G=S U(2)$.

One of the results of this work is to show the peakedness properties of the gauge-invariant coherent states. For the simplest example of a 1-flower graph this could be done analytically and showed interesting features. The gauge-invariant states are labeled by gauge orbits, and the overlap (4.29) between two gauge-invariant coherent states exhibits a peak structure at the point where both gauge orbits coincide. The width of this peak is proportional to the semiclassicality parameter $t$. Due to the fact that the space of the gauge orbits is no manifold (rather, it is an orbifold), the peakedness of the states is no clean Gaussian, but a more complicated function, that still tends to a Gaussian if $t$ goes to zero. This shows that states that are labeled by gauge orbits have useful semiclassical properties. In particular, the limit $t \rightarrow 0$ corresponds to the classical limit $a \gg \ell_{P}$ (3.9), in which the state approaches the classical gauge-invariant state determined by the gauge orbit. This stays true for states labeled at degenerate points of the orbifold, but in this case the state never approaches a Gaussian, not even for small $t$. Rather, the overlap exhibits a plateau that is much flatter than a Gaussian. In particular, the second derivatives at the maximum of the peak vanish along with all the odd derivatives, leading to a function that has a $\mathrm{e}^{-x^{4} / t^{2}}$ profile, rather than a Gaussian one.

Similar features could be established numerically for more complicated graphs, in particular the 2-flower, the 3-bridge and the tetrahedron graph. To investigate the overlaps on these graphs, we used a gauge-fixing procedure to separate the gauge-invariant degrees of freedom from those that are pure gauge. Not only did this show a lot about the general procedure how this can be done, but it also enabled us to work entirely with gauge-invariant variables, which made the peakedness properties for these states transparent. In fact, also on the 2-flower, the 3-bridge and the tetrahedron graph the peakedness properties for generic points of the space of gauge orbits could be seen. Additionally, the states on the 2-flower and the 3-bridge graph showed the same change in the peakedness structure at points that correspond to degenerate gauge orbits, i.e. orbits in $S L(2, \mathbb{C})^{E}$ under the gauge action $S L(2, \mathbb{C})^{V}$ whose dimension is less than $6 \mathrm{~V}$.

Apart from the special graphs, we have also derived some results for arbitrary graphs. First, we were able to prove that the 'flattening' of the overlap of states labeled at degenerate gauge orbits, which consist only of a point, is generic for $E$-flower graphs, i.e. for graphs with one vertex and $E$ edges all emerging and ending at that vertex. So this is not only a coincidence because of the simple graphs we have chosen, but rather this is true for any $E$-flower graph.

Second, we have generalized the gauge-fixing procedure that helped us in our numerical examples to extract the gauge-invariant information from the overlap expression for states on arbitrary graphs. This enabled us to establish a relation between the inner product of states on arbitrary graphs with $E$ edges and $V$ vertices with states on $(E-V+1)$-flower graphs. In particular, (5.20) shows this relation.

After that, we used this relation to extend the theorem about the peakedness properties of states labeled by degenerate gauge orbits from flower to arbitrary graphs. Unfortunately, in this case the theorem could only be established in the limit $t \rightarrow 0$, since only in this limit the expression became tractable. However, there are hints that the theorem is in fact true for 
finite $t$ as well, although the proof seems to be much harder than for the limit $t \rightarrow 0$. Still, the investigation of the second derivative asymptotics involves a careful taking of limits. Since there is no control over how fast these asymptotics approach zero, it would be desirable to have a result for arbitrary finite $t$.

Although the gauge-invariant coherent states labeled by degenerate orbits are not Gaussian peaked but have a peak that is much flatter, this does not spoil the semiclassical properties of gauge-invariant coherent states. First, the states are still peaked, the peak profile is just not the nice, clean Gaussian that one is used to from the harmonic oscillator coherent states or (approximately) from the complexifier coherent states on graphs. But still, the width of the peak is proportional to (a fractional power of) $t$, which indicates that the limit $t \rightarrow 0$ corresponds to the semiclassical limit, in which the state approaches a point in classical gauge-invariant phase space.

It is in fact not surprising at all that the peakedness of the states labeled at degenerate gauge orbits is qualitatively different. From a mathematical point of view the degenerate gauge orbits correspond to singular points ('edges' or 'corners') in the gauge-invariant phase space, which is, as already pointed out, no manifold. But also from a physical point of view this is not disturbing: The case, for instance, where all edge labels are $\mathbb{1} \in S L(2, \mathbb{C})$ corresponds to a state which is labeled by the physical distribution of the Ashtekar connection $A_{a}^{I}=0$ and $E_{I}^{a}=0$ along these edges $[2,18]$. So, this case corresponds to a highly degenerate metric. One could have guessed that these states exhibit a qualitatively different behavior than states that are labeled by elements which approximate, say, flat Minkowski space.

This feature will become important in the case of diffeomorphism-invariant coherent states, where there are many different types of degenerate gauge orbits, corresponding to symmetrical metric configurations. Since one is particularly interested in these situations (i.e. Minkowski space), one should expect peculiar peakedness properties for diffeomorphisminvariant coherent states labeled by configurations corresponding to these symmetric situations.

Another point investigated in this paper concerns the complexifier coherent states, and is of a more mathematical nature. For the inner product between two states a formula could be found that depends entirely on the geometry of the complexified gauge group. In particular, for $G=U(1)$ as well as for $G=S U(2)$, an expression could be derived that involves the complex lengths of geodesics on $G^{\mathbb{C}}$ (3.26). In particular, if both states are labeled on $G \subset G^{\mathbb{C}}$, then the inner product is a sum over all geodesics, involving terms proportional to Gaussians in the length of these geodesic measured by the Killing metric. For the case of $G=U(1)$ this was rather trivial and seemed to be a coincidence, while the corresponding formula for $G=S U(2)$ came more as a surprise. It raises the hope that a similar formula can be shown for CCS on arbitrary compact Lie groups $G$. There are in fact hints that support this conjecture.

Remember that the complexifier coherent states (choosing $\hat{C}=-\Delta$ as the complexifier) labeled by points on $G$ are nothing but solutions of the heat equation [10,3]. In particular, the norm of the CCS $\psi_{g}^{t}$ is equal to 1 as $g \in G$. Moreover, the inner product is given by

$$
\left\langle\psi_{g}^{t} \mid \psi_{g^{\prime}}^{t}\right\rangle=\psi_{\mathbb{1}}^{2 t}\left(g^{-1} g^{\prime}\right)
$$

so it also solves the heat equation (in $t$ ). But the heat equation can be thought of releasing a random walker at $\mathbb{1} \in G$, letting it walk along $G$ for some finite time $t$, and then measure the probability distribution $\rho(t, g)=\psi_{\mathbb{1}}^{t}(g)$ of where he is on $G$. Obviously, the smaller the allowed time $t$ or farther the geodesic distance (defined by the Killing metric on $G$, which is positive definite) between $\mathbb{1}$ and $g$, the smaller the probability $\rho(t, g)$. In particular, on $\mathbb{R}^{n}$ this probability is given by a Gaussian in the geodesic distance, which might also be true on arbitrary $G$. Furthermore, the sum over all geodesics from $\mathbb{1}$ to $g$ arises naturally, since it 
encodes the different ways the random walker could have taken on $G$ to walk from $\mathbb{1}$ to $g$, since the topology of $G$ will, in general, be nontrivial.

These considerations lead to the possibility that one could be able to define a generalization of (3.26) to arbitrary compact Lie groups. We hope to be able to address this point in some future work.

\subsection{Conclusion and outlook}

In [1] and the present paper, we have shown that the kinematical complexifier coherent states, which are convenient tools for investigating the semiclassical limit of the kinematical sector of LQG, can be projected to the gauge-invariant subspace, and the resulting gauge-invariant coherent states are suitable for addressing semiclassical issues on the gauge-invariant sector.

It may seem quite discouraging that the Gauss-invariant coherent states for LQG considered here are difficult to handle analytically. However, one should keep in mind that in this paper we only investigated the integral formula (4.20) rather than the sum over intertwiners such as (4.19). It may well be that using asymptotic formulae for large spin for Clebsch-Gordan coefficients, $6 j$-symbols, etc (see, e.g., [21-23]) one can gain more analytical control. Also it may be that we overlooked some clever technique that allows us to simplify the gauge integrals. We hope to come back to this point in some future publication. In any case, numerically the Gauss-invariant states are well under control, although one needs to write an adapted code to handle arbitrary complicated graphs.

The next obvious step will be to consider the action of the diffeomorphism group on the set of (gauge-invariant) coherent states. In particular, the projection of the gauge-invariant coherent states to the diffeomorphism-invariant Hilbert space $\mathcal{H}_{\text {diff }}$ via some sort of rigging map, in order to arrive at diffeomorphism-invariant coherent states. This space is-other than the gauge-invariant Hilbert space-not completely under control, in particular, there is no unique definition of $\mathcal{H}_{\text {diff }}[4,7]$. So, this task will be significantly more challenging than theconceptually quite clear-definition of the gauge-invariant coherent states. But this could grant a way to investigate all the different possibilities to define $\mathcal{H}_{\text {diff }}$, and maybe even distinguish some of them as more suitable than others. Furthermore, as soon as the diffeomorphisminvariant coherent states are defined, approximations and semiclassical techniques for the graph-changing version of the master constraint $[19,20]$ become available, which encode the Hamiltonian constraints and can be defined on $\mathcal{H}_{\text {diff }}$.

\section{Acknowledgments}

BB would like to thank Christian Bär for knowledge about cohomology classes, Bianca Dittrich for the discussion about degenerate gauge orbits, Hendryk Pfeiffer for the support and discussions, and Klaus Wirthmüller for his time and patience. Also, we thank the referees for many useful comments to the original manuscript. Research at the Perimeter Institute for Theoretical Physics is supported by the Government of Canada through NSERC and by the Province of Ontario.

\section{References}

[1] Bahr B and Thiemann T 2009 Gauge-invariant coherent states for loop quantum gravity: I. Abelian gauge groups Class. Quantum Grav. 26045011 (arXiv:0709.4619)

[2] Thiemann T 2001 Gauge field theory coherent states (GCS): I. General properties Class. Quantum Grav. 182025 (arXiv:hep-th/0005233) 
[3] Thiemann T and Winkler O 2001 Gauge field theory coherent states (GCS): II. Peakedness properties Class. Quantum Grav. 182561 (arXiv:hep-th/0005237)

Thiemann T and Winkler O 2001 Gauge field theory coherent states (GCS): III. Ehrenfest theorems Class. Quantum Grav. 184629 (arXiv:hep-th/0005234)

[4] Thiemann T 2006 Introduction to Modern Canonical Quantum General Relativity (Cambridge Monographs on Mathematical Physics) (Cambridge: Cambridge University Press)

[5] Rovelli C 2004 Quantum gravity Cambridge Monographs on Mathematical Physics (Cambridge: Cambridge University Press)

[6] Smolin L 2004 An invitation to loop quantum gravity, in Quantum Theory and Symmetries: Proc. 3rd Int. Symp. (Cincinnati, OH, 10-14 Sept 2003) ed P C Argyres, T J Hodges, F Mansouri, J J Scanio, P Suranyi and L C R Wijewardhana (Singapore: World Scientific) pp 655-82 (arXiv:hep-th/0408048)

[7] Ashtekar A, Lewandowski J, Marolf D, Mourao J and Thiemann T 1995 Quantization of diffeomorphism invariant theories of connections with local degrees of freedom J. Math. Phys. 36 6456 (arXiv:gr-qc/9504018)

[8] Lewandowski J, Okołow A, Sahlmann H and Thiemann T 2006 Uniqueness of diffeomorphism invariant states on holonomy-flux algebras Commun. Math. Phys. 267703 (arXiv:gr-qc/0504147)

[9] Rovelli C and Smolin L 1995 Spin networks and quantum gravity Phys. Rev. D 525743 (arXiv:gr-qc/9505006)

[10] Hall B 1994 The Segal-Bargmann 'coherent state' transform for compact Lie groups J. Funct. Anal. 122103 Hall B 1997 The inverse Segal-Bargmann transform for compact Lie groups J. Funct. Anal. 14398

[11] Hall B 1997 Phase space bounds for quantum mechanics on a compact Lie group Commun. Math. Phys. 184233

[12] Giesel K and Thiemann T 2007 Algebraic quantum gravity (AQG): I. Conceptual setup Class. Quantum Grav. 242465 (arXiv:gr-qc/0607099)

Giesel K and Thiemann T 2007 Algebraic quantum gravity (AQG): II. Semiclassical analysis Class. Quantum Grav. 242499 (arXiv:gr-qc/0607100)

Giesel K and Thiemann T 2007 Algebraic quantum gravity (AQG): III. Semiclassical perturbation theory Class. Quantum Grav. 242565 (arXiv:gr-qc/0607101)

[13] Flori $\mathrm{C}$ and Thiemann $\mathrm{T}$ Semiclassical analysis of the loop quantum gravity volume operator utilising cylindrically consistent complexifier coherent states (in preparation)

[14] Livine E R and Speziale S 2007 A new spinfoam vertex for quantum gravity Phys. Rev. D 76084028 (arXiv:0705.0674)

Livine E R and Speziale S 2008 Consistently solving the simplicity constraints for spinfoam quantum gravity Europhys. Lett. 8150004 (arXiv:0708.1915)

[15] Freidel L and Krasnov K 2008 A new spin foam model for 4d gravity Class. Quantum Grav. 25125018 (arXiv:0708.1595)

[16] N. Biggs 1993 Algebraic Graph Theory 2nd edn (Cambridge: Cambridge Mathematical Library)

[17] Freidel L and Livine E 2003 Spin networks for non-compact groups J. Math. Phys. 441322 (arXiv:hep-th/0205268)

[18] Thiemann T 2001 Complexifier coherent states for quantum general relativity Class. Quantum Grav. 182025 (arXiv:gr-qc/0206037)

[19] Thiemann T 2006 The Phoenix Project: master constraint programme for loop quantum gravity Class. Quantum Grav. 232211 (arXiv:gr-qc/0305080)

[20] Thiemann T 2006 Quantum spin dynamics: VIII. The master constraint Class. Quantum Grav. 232249 (arXiv:gr-qc/0510011)

[21] Baez J, Christensen D and Egan G 2002 Asymptotics of $10 j$ symbols Class. Quantum Grav. 196489-513 (arXiv:gr-qc/0208010)

[22] Barrett J W and Steele C M 2003 Asymptotics of relativistic spin networks Class. Quantum Grav. 20 1341-62 (arXiv:gr-qc/0209023)

[23] Freidel L and Louapre D 2003 Asymptotics of $6 j$ and $10 j$ symbols Class. Quantum Grav. 20 1267-94 (arXiv:hep-th/0209134) 